\title{
Health Professionals' Perceptions and Experiences of Open Disclosure: A Systematic Review of Qualitative Evidence.
}

\author{
Jacinta Byrth \\ Master of Clinical Science \\ Joanna Briggs Institute \\ Faculty of Health Science \\ The University of Adelaide \\ Australia
}

September 2013 


\section{Abstract}

Background: In 1999 the Institute of Medicine released its seminal report 'To Err is Human; Building a Safer Health System', drawing worldwide attention to the issue of patient safety in healthcare. Internationally there is a move towards open disclosure as a standard for professional health care practice. It has been suggested that open disclosure plays a pivotal role in achieving a high level of quality of health care and patient safety, as transparency and discussion about errors builds trust between the patient and the health professional and health system. However, studies suggest that there is a significant gap between the level of endorsement of open disclosure of medical errors to patients by health professionals in theory and the actual level experienced by patients in reality.

Objective: To synthesise the best available research evidence exploring health professionals' perceptions and experiences of the barriers and facilitators of open disclosure of medical errors to patients.

Design: A systematic review of the qualitative evidence using The Joanna Briggs Institute meta-aggregative approach to qualitative evidence synthesis.

Results: Full text review of 41 papers, with the inclusion of nine (9) papers following critical appraisal by two (2) reviewers. A total of 131 findings were extracted and aggregated into 33 categories. Final meta-synthesis generated two (2) key findings; the barriers to open disclosure included personal fears, professional factors, error factors, patient factors, cultural factors, system related and uncertainties; facilitators to open disclosure include professional factors, error factors, system factors, cultural factors and personal needs.

Conclusion: The factors impacting on open disclosure by health professionals are varied and complex. Evidence shows a structured approach to the disclosure of errors to patients can assist in removing barriers and enhancing facilitators. Further research 
is required to investigate the patients' perspective to ensure the process of disclosing errors is appropriate and patient centered. 


\section{Acknowledgments}

I would like to thank my primary supervisor, Associate Professor Edoardo Aromataris, and my secondary supervisor Ms Alexa McArthur for their assistance and support in completing this Thesis.

I would also like to thank Dr Suzanne Robertson-Malt as my secondary reviewer and Ms Maureen Bell, research Librarian for helping me with the development of a comprehensive search strategy.

I would also like to thank Annabel, Felicity and Nicholas for their unconditional love and support. 


\section{Declaration}

I, Jacinta Byrth, certify that this work contains no material that has been accepted for the award of any other degree or diploma in any university or any other tertiary institution, and, to the best of my knowledge and belief, contains no material previously published or written by any other person, except where due reference has been made in the text. In addition, I certify that no part of this work will, in the future, be used in a submission for any other degree or diploma in any university or other tertiary institution without the prior approval of the University of Adelaide and where applicable, any partner institution responsible for the joint-award of this degree.

I give consent to this copy of my thesis, when deposited in the University Library, being made available for loan and photocopying, subject to the provisions of the Copyright Act 1968.

I also give permission for the digital version of my thesis to be made available on the web, via the University's digital research repository, the Library catalogue, and also through web search engines, unless permission has been granted by the University to restrict access for a period of time.

Jacinta Byrth

$30^{\text {th }}$ September 
Table of Contents

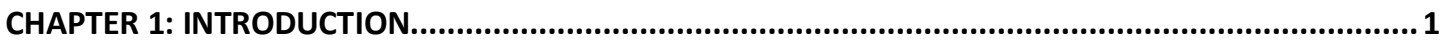

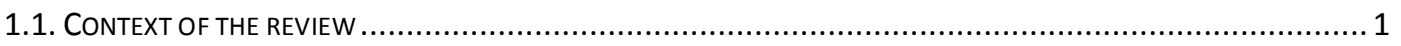

1.2. RECENT DEVELOPMENT AND IMPLEMENTATION OF AN OPEN DISCLOSURE POLICY...................................8

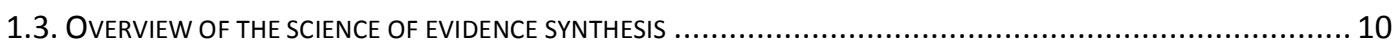

1.4. METHOdOLOGICAL BASIS FOR THE REVIEW ................................................................. 14

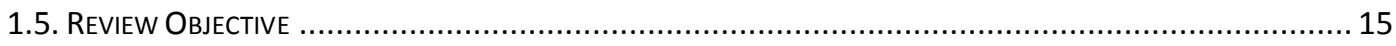

1.6. ReVIEW QUESTION ........................................................................................... 15

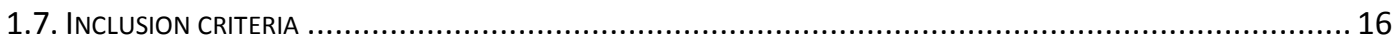

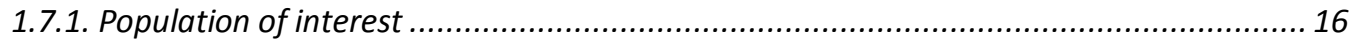

1.7.2. Phenomena of Interest................................................................... 16

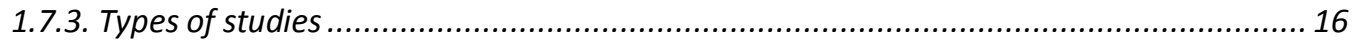

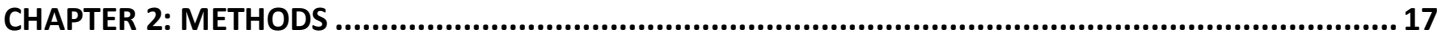

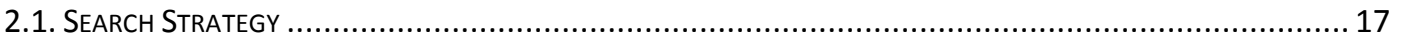

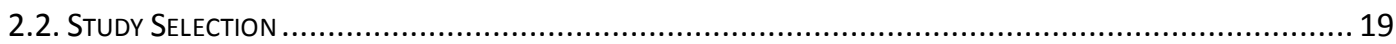

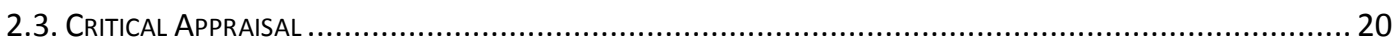

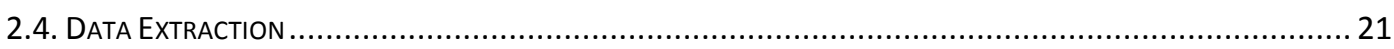

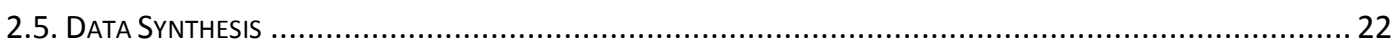

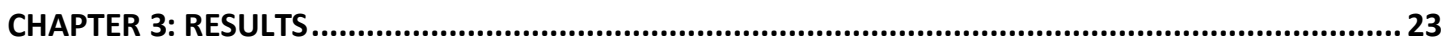

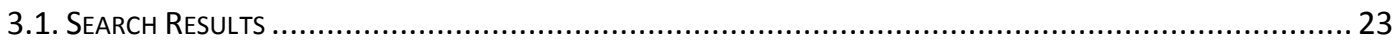

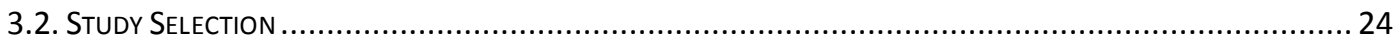

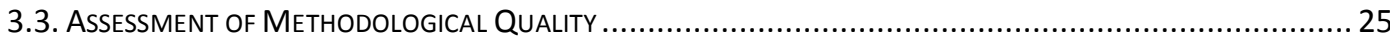

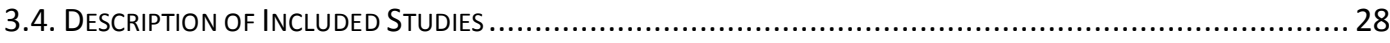

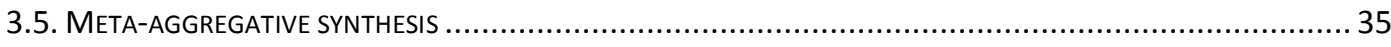

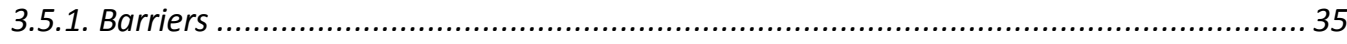

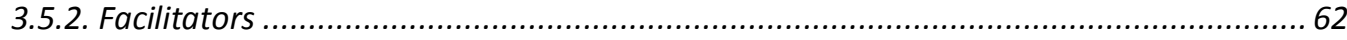

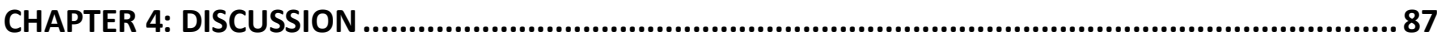

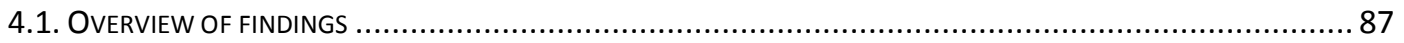

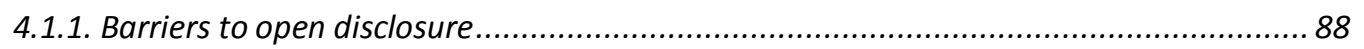

4.1.2. Facilitators of open disclosure ................................................................. 94

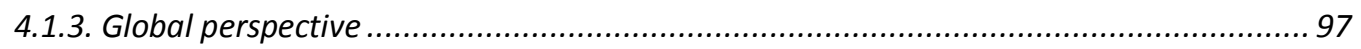


4.2. LIMITATION TO THE STUDY

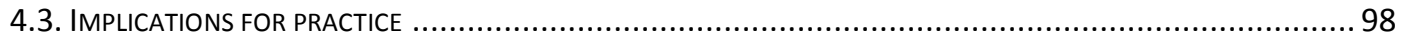

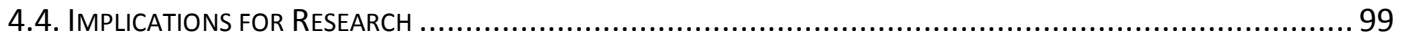

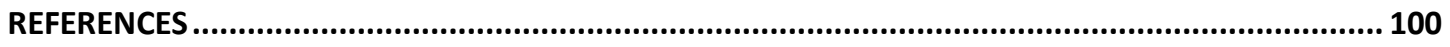

APPENDIX I: DETAILED DATABASE SEARCH STRATEGY ......................................................... 110

APPENDIX II: QARI APPRAISAL INSTRUMENT ................................................................ 116

APPENDIX III: QARI DATA EXTRACTION INSTRUMENT.......................................................... 117

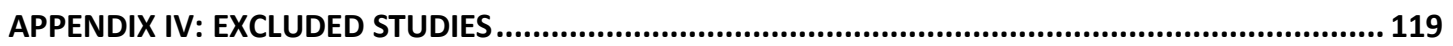




\section{Chapter 1: Introduction}

\subsection{Context of the review}

In 1999 the Institute of Medicine (IOM) released its seminal report 'To Err is Human: Building a Safer Health System', drawing worldwide attention to the issue of patient safety in healthcare (Kohn et al., 2000). The IOM reported that up to 98,000 people die in hospitals across the United States of America each year as a result of medical error, with an estimated additional cost of US $\$ 17$ billion to $\$ 29$ billion at that time, to the annual health care budget. The 'To Err is Human' report exposed not only the financial and human costs of medical errors, the latter illustrated by the increasing mortality rates, but went further to identify these types of errors in health care as a chronic threat to public health (Kohn et al., 2000).

The ethical imperative "first do no harm" has been a fundamental principle of the medical profession since Hippocratic times (Hevia and Hobgood, 2003; Waite, 2005; Moskop et al., 2006). However, health care by its very nature is intrinsically risk laden and potentially hazardous for patients, owing in part to the fact it is a complex enterprise delivered by multidisciplinary teams of health professionals. Despite the best intentions of health professionals and health care organisations, mistakes are universal to the human experience and can occur at all levels of personal and professional life (Matlow et al., 2006; Gallagher et al., 2007b). A medical error is considered to be a preventable adverse event where a patient is harmed during the course of care, as a consequence of either what has been done to them - an error of commission, or conversely, what has not been done, an error of omission (Hevia and Hobgood, 2003; Chamberlain et al., 2012).

Providing patients with information after any unanticipated outcome arising as a result of their care is an established ethical expectation for all health professionals (Eaves-Leanos and Dunn, 2012). The Ethics Manual of the American College of Doctors states that rules for truth telling precedes the principle of respect for patient 
autonomy (Hebert et al., 2001; Rowe, 2004; Moskop et al., 2006; Eaves-Leanos and Dunn, 2012). Historically, healthcare decisions surrounding the appropriateness, timing and content of medical information provided to patients has remained a private matter, which has been left to the preferences and discretion of individual clinicians and health care institutions (Lamb et al., 2003; Garbutt et al., 2008). The literature suggests the development of a systematic framework for health professionals to respond to medical errors and provide information regarding medical errors to patients, supports the notion of quality health care (Gallagher et al., 2007a; Australian Commission on Safety and Quality in Health Care, 2008) Adoption of a structured process promotes a collaborative and cohesive approach to the management of errors and furthermore, guides health professionals on what immediate steps to pursue when an error occurs. Such a structured approach also provides strategies to support their interactions with the involved patient and their family (Kaiser Family Foundation, 2005).

Open disclosure is defined as providing an open, consistent approach to communicating with patients following an adverse event that has occurred during the course of their care (Conway et al., 2011; Eaves-Leanos and Dunn, 2012). Open disclosure describes the way clinicians communicate with patients (including family members or carers) who have experienced harm as a result of the health care they received. The purpose is to assist patients who have experienced harm, guide the clinicians, the clinical workforce and health service organisations in supporting patients who have experienced harm. The process promotes transparency, and provides an opportunity for the health service organisations to learn from adverse events to prevent recurrences.

Open disclosure is a complex and challenging process, it requires courage, composure, communication skills and a fundamental belief the patient is entitled to know the truth (Boyle et al., 2006). It pushes the traditional practice of sharing medical-clinical knowledge with patients to new limits and such conversations require specific skills. It is very difficult to initiate and have conversation that are 
likely to raise issues of failure, harm, loss of trust as these elements all have the potential to generate a wide spectrum of intense emotions including anxiety, anger, depression and guilt (Kaldjian et al., 2006b; Madden and Cockburn, 2007).

It has been suggested the practice of open disclosure plays a pivotal role in patient safety, since transparency and discussion about errors builds trust between the patient the health professional and the health system (Lamb et, al., 2003; Leape, 2006; Abholz et al., 2008). It also provides opportunities to identify and reflect on the contributing factors that lead to the error and to focus on what needs to be addressed and improved to prevent a recurrence (Manser and Staender, 2005). Moreover, the implementation and compliance with open disclosure policies in practice facilitates the flow of critical information and provides an organisation with vital opportunities to examine medical errors and the circumstances in which they occurred (Manser, 2011). Furthermore, it provides opportunities to translate the lessons learnt from medical errors into concrete changes to improve practices and thereby reducing the risk of harm to others (Manser and Staender, 2005; MacDonald and Attaran, 2009; Manser, 2011). In 2001, the Joint Commission on Accreditation of Healthcare (JCAHO) endorsed open disclosure as an effective patient safety strategy with the introduction of a new standard requiring Joint Commission accredited hospitals to inform patients of all unanticipated outcomes of care that they experience (Fein et al., 2007).

Similarly, across health care systems internationally, there is a move towards adoption of open disclosure as a standard for professional healthcare practice. Several countries have mandated polices in direct response to increasing rates of litigation claims associated with medical errors. Open and effective communication with patients about errors is considered to reduce the number of litigation claims (Fein et al., 2007). Investigations into this area of litigation suggest the motivation for patients to pursue legal action following a medical error is not simply because they believe there has been negligence, but because of a breakdown in communication with their providers (Mariner, 2001; Winslade and McKinney, 2006, Studdert and Richardson, 
2010). Patients who believe they are not being told everything surrounding an error that has occurred during their care will often seek to initiate legal action as an opportunity to simply learn the details of what actually happened (Witman et al., 1996; Vincent, 1998; Clinton and Obama, 2006).

Patient centred models of care are recognised as being ideal for effective, quality health care delivery by meeting the needs of the patient (Conway et al., 2011; Iedema et al., 2011a). Patients are increasingly becoming more involved in their own health care needs and are developing active partnerships with health professionals to participate in the planning and delivery of health care to achieve their individual health care goals (Eaves-Leanos and Dunn, 2012). Consequently, there is a repositioning of the balance between medically or professional dominated approaches to care decision-making and consideration for the particular needs of the individual patient. Health professionals face new challenges with this shift towards patient centred care; they are required to reflect and consider behaviour to promote the best interest of the patient, including maximising patient benefits while minimising patient harms (Sorensen et al., 2008). The fundamental principles of patient centred care are ensuring that all clinical decisions are respectful of, and responsive to, the individual patients' preferences, needs and values (Gallagher et al., 2006a; O'Connor et al., 2010; Conway et al., 2011).

To identify the needs of patients surrounding the disclosure of medical error, studies have examined the expectations of patients and families following an adverse event in the course of their care (Manser and Staender, 2005; Levinson, 2009). The findings suggest that patients both expect and desire open disclosure of medical errors; in short, they want an explanation of what transpired. Furthermore, it is clear patients also want to be told and reassured that the health care organisation has learnt from these errors and actively moves forward with identifying and implementing effective measures to prevent similar errors from happening to others in the future (Mazor, 2004; Schwappach and Koeck, 2004; Gallagher and Levinson, 2005; Kaiser Family Foundation, 2005). Despite the ethical obligation to disclose errors being widely 
acknowledged, there is evidence to suggest such disclosure is both inconsistent and often uncommon in practice and it continues to be a controversial issue amongst some health professionals (Gallagher et al., 2007b; Levinson, 2009).

Studies suggest that there is a significant gap between the level of endorsement of open disclosure of medical errors to patients by health professionals in theory and the actual level of disclosure experienced by patients in reality (Waite, 2005; Fein et al., 2007; Iedema et al., 2011b). To examine the reasons for this gap, studies examining the open disclosure of medical errors to patients and the behaviours of physicians, suggest there are a variety of factors that impinge upon their readiness to disclose medical errors to patients (Kaldjian et al., 2006b). These include the fear of litigation, a strong cultural reluctance within medical practice to admit mistakes, and a fear by practitioners they would lose the support of their colleagues or the organisation in which they practice (Gallagher et al., 2007b).

Research has also revealed that physicians fear that by informing patients about a mistake they would risk damaging or losing their personal and professional reputation and any future career opportunities (Gallagher et al 2006a; Gallagher et al 2007b). Physicians studied also expressed belief that patients would not want to know if an error had occurred. Furthermore, if they were to inform patients about an error, it was unlikely the patient would understand the information or details of what they were being told (Gallagher et al. 2006b). Moreover, physicians were less likely to disclose an error if they believed the patient was unaware an error had actually taken place or if there was no obvious level of harm to the patient (Hingorani et al., 1999). The literature also suggests other health professionals besides physicians fail to disclose errors for several reasons. These reasons for non-disclosure were out of a desire to protect patients and their family from any additional anxiety and distress, to prevent undermining the patient's trust in the care that they are receiving, and also to protect their own relationship with the patient and their family (Crigger, 2004; O'Connor et al., 2010; Sorensen et al., 2010). 
The relationship between health professionals and patients is built on a foundation of trust (Sorensen et al., 2010). Emotions generated and evoked in patients by their illness can also have a significant impact on the professional-patient relationship (Smith and Forster, 2000). Within the clinical encounter with the health professional, some patients may choose to reveal and talk about highly emotionally charged issues, placing their relationship with the health care professional on a level that is often viewed to have developed to being personal, caring and respectful (Smith and Forster, 2000). Many patients feel vulnerable by the virtue of being unwell or by being in the situation where they need to seek medical care. Therefore, having trust in the health professionals involved in the development and delivery of a treatment plan can promote the process of healing (Iedema et al., 2007; Shannon et al., 2009).

Professional virtues or particular character traits amongst health care staff also support the establishment of trust between the patient and health professional; the virtue of truthfulness is the habit of telling the truth even when it is inconvenient or involves some personal risk (Fein et al; 2005; Greene, 2009). Trust is ultimately essential for an effective professional-patient relationship because relationships cannot endure lack of truthfulness (Smith and Forster, 2000). Following an incident, effective communication that is open and honest is vital to preserve and restore trust in the relationship. It is also necessary to facilitate appropriate treatment actions in response to any error and the patient's ongoing care (Hannawa et al., 2013; Conway et al., 2011).

The health professional-patient relationship often becomes complicated in the aftermath of an error. When a patient is harmed unintentionally, by the very people they entrusted to help them, it can create feelings of disillusionment and failed expectations (Boyle et al., 2006; Harvard, 2006). An incident or error in their treatment represents a major threat to the patient's sense of control and trust in both the caregiver and the health care system. The relationship between the patient and the health professional is put under further strain in situations where the health professional who was actually involved in the error may be required to continue to 
provide ongoing care for the patient (Manser, 2011). This places the patient in a difficult situation and they can often experience conflicting emotions about not only the health professionals but also the entire health service (Harvard, 2006; Conway et al., 2011). The patient and their family members' reactions to an incident are influenced by not only by the nature of the incident and severity of the outcome, but also by the response, acknowledgement of the incident, communication about the incident and the overall management of the situation (Australian Commission on Safety and Quality in Health Care, 2013; Conway et al., 2011). The failure to acknowledge an event can be very distressing for the patient and can be a powerful stimulus for patients to lodge official complaints or to ultimately pursue legal action (Mariner, 2001; Harvard, 2006).

Conversely, although in the case of a medical error the patient and family members are considered to be the obvious victims, it is less well recognised that the health professional involved in the incident often becomes the second victim (Wu, 2000; Edrees et al., 2011). Second victims are defined as health care providers who are involved with a patient-related adverse event or medical error, and as a result, experience emotional and sometimes physical distress (Wu, 2000). In addition to feelings of shame, guilt and fear, these second victims may doubt their clinical competence and even their ability to continue working as a provider of health care. In a medical culture in which errors pose risks to performance evaluations and liability claims, it can be difficult for second victims to seek emotional support. A study by Edrees et al., 2011 looked at the importance of support structures for second victims. The study involved 350 participants from various health professionals and institutions within John Hopkins Medical Centers in the USA.

Study results reflect the need of a professional environment where errors can be openly discussed and examined without fear of blame and negative consequences (Edrees et al., 2011). The management of medical errors in practice can have a significant long term effect on the perceptions, confidence, self-esteem and long term attitudes of staff, especially of novice nurses, medical students and junior doctors 
who are considered to be most vulnerable and impressionable during their early stages of professional development (Wu et al., 1991; Crigger, 2004; Gunderson et al., 2009; Shannon et al., 2009).

\subsection{Recent development and implementation of an open disclosure policy}

Historically, the open disclosure of medical errors to patients has primarily been considered a risk management issue, with concerns of potential legal risk associated with informing patients about medical errors (Clinton and Obama, 2006). However, in recent years a broader view has been taken to find solutions for the effective management of medical errors, and open disclosure has been recognised internationally as a practice that can be beneficial for both patients and clinicians involved in medical errors (Conway et al., 2011). Although it is inherently complex and difficult for everyone involved, its systematic practice can assist health service organisations to manage adverse events compassionately and provide broader benefits through improved clinical communication and systems improvement (Australian Commission on Safety and Quality in Health Care, 2013).

In 2001, the JCAHO introduced a standard that required health care facilities in the USA accredited by the Joint Commission to inform patients of all unanticipated outcomes of care that they experienced (Fein et al., 2007). In addition to this accreditation requirement some states in the USA implemented laws requiring health care organisations to disclose adverse events of unanticipated outcomes to patients (Iedema et al, 2011a). Furthermore, in 2003 the American Society for Healthcare Risk Management (ASHRM) released a three part series to provide perspectives of those working on the front lines of disclosure development and implementation (American Society for Healthcare Risk Management, 2003). This series covered three major concepts: improving communication, the influence of litigation, what works now and what can work better. 
In 2003, the Health Ministers' from the states and territory governments of Australia supported disclosure of medical errors to patients with the endorsement of the Open Disclosure Standard; this was the first nation wide-open disclosure policy in the world. Following its release, some years later in 2011/2012 it was reviewed; from this review the current Australian Open Disclosure Framework was developed and endorsed by the Australian Commission on Safety and Quality in Health Care in 2013. The Open Disclosure Framework is designed to enable health service organisations and clinicians to follow a process to facilitate open communication with patients, provide a structure on how it should occur and what components will assist the process when health care does not go to plan. Providing a clear framework provides a nationally consistent basis for open disclosure of medical errors to patients in Australian health care (Australian Commission on Safety and Quality in Health Care, 2013).

In 2010 the Institute for Healthcare Improvement (IHI) published a white paper on “Respectful Management of Serious Clinical Adverse Events” (Conway et al., 2011). The report revealed that many health care organisations do not have a planned response for responding and managing adverse events. It was identified that an organisation has three specific priorities following an adverse event. The first priority identified was the need to provide immediate care for the patient and his or her family members who are the direct victims of the adverse event. The second priority identified was to provide care for the frontline clinicians who had been involved in or exposed to the adverse event. In the report these individuals were referred to as second victims, a term that was first introduced by the work of Wu in 2000 (see above). The third priority of the response plan was to address the needs of the organisation, which can also suffer a potential loss from the incident, becoming a third victim.

Similarly, in 2010 the National Quality Forum (NQF) released A Consensus report, Safe Practices For Better Health Care. Safe Practice \# 7- Disclosure, outlines the objective to provide open and clear communication with patients' and their families 
about serious unanticipated outcomes that are supported by systems that foster transparency and performance improvement to reduce preventable harm (National Quality Forum, 2010). In 2012, the release of the Veterans Health Administration (VHA) directive "Disclosure of Adverse Events to Patients" supports their commitment to disclose the occurrence of harmful adverse events to patients. This is consistent with the VHA core values of integrity, commitment, advocacy, respect, and excellence, demonstrating respect for the patient and professionalism and a foundation for improving care (Department of Veteran Affairs, 2008). It also is consistent with The Joint Commission (TJC) standard, that requires patients, and when appropriate their families, be told of "unanticipated outcomes" of care. The VHA believes that there is an unwavering ethical obligation to disclose harmful adverse events to patients that have occurred in the course of their Department of Veterans Affairs (VA) care, including cases where the harm may not be obvious, or where there is a potential for harm to occur in the future (Department of Veteran Affairs, 2008).

Providing safe, patient focused care is a major challenge confronting today's increasingly complex health care system. Open disclosure represents a new dimension of accountability and challenges the traditional hierarchical values and practices of health professionals (Iedema et al., 2009). The literature suggests open disclosure has beneficial consequences for patient safety; it supports the patient's right to be informed when something goes awry during their care and promotes the virtues of honesty and humility amongst health professionals (Cravens and Earp, 2009; Piper and Iedema, 2011).

\subsection{Overview of the science of evidence synthesis}

Open disclosure is an important aspect of clinical practice and is increasingly acknowledged as the appropriate response for health professionals to take following a medical error. It is a challenge for health professionals, for several reasons, firstly the traditional and previously acceptable ways of dealing with errors are being 
questioned, and patients' preferences and expectations are having a greater influence on how organisations manage medical errors and open disclosure.

Policy makers and practitioners are gradually becoming aware of the limitations of systematic reviews of high quality randomised controlled trials as the exclusive evidence base for informing practice. Therefore, meta-synthesis of qualitative research has increased in significance with the demand for research that recognizes the importance of patient perspectives, complexity and settings (Walsh and Downe, 2005). A systematic review of the current qualitative evidence lends itself to gain a deeper level of inquiry to investigate what are the barriers and facilitators that have an impact on health professionals decision whether to disclose errors to their patient or not. This richness of qualitative data is recognized as providing credible, complementary findings, which can be drawn together in a synthesis and used to inform practice and assist policy makers (Pearson, 2004; Pearson and Jordan, 2007; Hannes and Lockwood, 2011).

Historically, in healthcare what is considered evidence has been dominated by the positivistic paradigm. The randomised controlled trial has been viewed as the gold standard of quantitative research and evidence with the synthesised results from well conducted randomised controlled trials used to guide the clinical decision making process (Hannes et al., 2005). However, the richness of data and evidence generated from qualitative research methods provides a deeper understanding of how things connect and interact in the complex field of healthcare (Hannes and Lockwood, 2011). Qualitative research enables health sciences researchers to delve into questions of understanding, meaningfulness and feasibility of health issues, and to examine institutional, social practices and processes (Pearson, 2004).

Common methodologies for qualitative research include, ethnography, phenomenology, grounded theory, and feminist inquiry (The Joanna Briggs Institute, 2011). In healthcare, qualitative research methodologies allow the researcher to identify the barriers and facilitators to the implementation of change and help to 
discover the reasons for the success or failure of implemented interventions. One major approach towards furthering the understanding of barriers and facilitators is phenomenology; it is a descriptive study of how individuals experience a phenomenon and enables the researcher to investigate the meaning, structure and essence of the lived experience of the phenomenon of interest by an individual or by many individuals (Giorgi, 2005; Dowling, 2007). The researcher is able to investigate the layers of the individuals' world of experience, which is considered to be where true consciousness exists. Phenomenological researchers often search for commonalities across individuals rather than only focusing on what is unique to a single individual (Koch, 1999; Giorgi, 2005; Pearson et al.; 2007). By asking participant questions to generate discussion and dialogue of their own personal experience it provides an opportunity for the researcher to gain insight into the participants' feelings and emotions surrounding their experience (Manen, 1990; Borbasi, 1996). Furthermore, providing a forum for participants with similar experiences to come together creates a richness of information that cannot be achieved through quantitative methodologies (Dowling, 2007).

Findings derived from qualitative research methodologies arise from the analysis of data that has commonly been generated from interviews or observations of participants or from document analysis (The Joanna Briggs Institute, 2011). Some of the methods employed to analyse this data are, content analysis, comparison, identification of themes, ethnographic methods, and discourse analysis (Thorne, 2010). Qualitative interviews place an emphasis on listening and following the direction of the participant. The participant provides the researcher with information through verbal interchange or conversation with a variety of chosen open-ended questions to elicit the most information possible from the participant within the time that is available. Interviews may be conducted with individuals or with focus groups. Focus groups are a formal method of interviewing a group of people/participants on a topic of common interest (The Joanna Briggs Institute, 2011). This method is useful for uncovering experiences, perceptions, attitudes and beliefs that dictate behaviour by the group members sharing their experiences (Doody, et al., 2013). By creating an 
environment where all participants feel at ease the probability of a candid discussion of even sensitive subject matter is greatly enhanced and provides a rich source of otherwise unobtainable information.

Conversely, the primary objective of quantitative methods of research is to quantify data and provide a result from a sample of the population of interest. Surveys and questionnaires can be used to measure the incidence of various views and opinions in a chosen sample (The Joanna Briggs Institute, 2011). The findings from surveys and questionnaires offer specific information, so the reliance on data from surveys and questionnaires would present great difficulty in gaining an understanding of the underlying deeper meaning linked to the responses. Therefore, in this case such methods would not be suitable to answer the questions posed in this systematic review.

Since the late 1980s researchers with a qualitative background have been developing methods to synthesise findings from qualitative research to provide credible information that can be used by practitioners and policy makers (Hannes and Lockwood, 2011). Primary qualitative research does not aim to be generalisable, however, via the aggregation process of combining findings, primary qualitative research can be used to assemble broad synthesised statements related to the topic examined (Thorne, et, al., 2004). It is an interpretive process but requires transparency of process and requires the reviewers to identify and extract the findings from papers included in the review. The findings are then categorised and aggregated into categories to develop synthesised findings, in this way meta-synthesis can provide both in-depth and broad coverage of the topic (Pearson et al., 2005, Kinn et al., 2013).

Despite the various methods of meta-aggregative synthesis of qualitative research findings, such as meta-ethnography, meta-study, thematic synthesis, critical interpretive synthesis, content analysis, and grounded theory aligning with different epistemological frameworks or paradigms, all seek to emphasize the complexity of 
interpretive and critical understandings of phenomena. Across all qualitative syntheses the phenomenon of interest to be studied must first be conceptualised based on the interest of the researcher, gaps in the current research or in response to predetermined priorities set by a professional organisation or by policy makers. The method of synthesising findings can vary; some methods would promote a very linear approach to synthesis while other methods such as meta-ethnography and grounded theory would follow a more iterative process (Hannes and Lockwood, 2011).

\subsection{Methodological basis for the review}

This systematic review utilised The Joanna Briggs Institute (JBI) Systematic Review method of qualitative synthesis and followed a structured process to achieve synthesised findings from the included studies. A particular feature of the JBI metaaggregative approach is that it has been designed to move beyond the production of theory to produce statements in the form of 'lines of action' (close to recommendations) likely to be undertaken and generally directive in nature (Hannes and Lockwood, 2011).

The JBI systematic review method requires a series of steps, which have been followed as outlined in this thesis. The steps are:

- Development of a rigorous protocol including an explicit research question;

- $\quad$ Clearly stated inclusion and exclusion criteria;

- $\quad$ A detailed search strategy designed to find all relevant studies;

- Critically appraising studies that meet the inclusion criteria;

- Extracting data from the primary studies including details of the participants, phenomena of interest, the study setting and key findings; and

- $\quad$ Pooling of the findings of at least two or more studies (Pearson et al., 2005).

The protocol for this systematic review was developed and presented to a panel of experts in the field for review in June 2012. The panel meeting provided an opportunity for clarification of any aspects of the protocol and discussion about the 
topic. Recommendations were provided for consideration to refine the systematic review protocol prior to commencement of the review (Byrth et al., 2012).

\subsection{Review Objective}

The objective of this systematic review was to synthesise the best available qualitative evidence exploring health professionals' perceptions and experiences of open disclosure of medical errors to patients.

This systematic review is important to gain collective insight into the contextual factors that assist and also those that hinder open disclosure. It addresses this important phenomenon using a comprehensive search strategy to locate the evidence, assessment of the quality of the primary research, and synthesising the findings extracted from the best available evidence on this topic. It is anticipated that the results of this systematic review of the available research on this issue will assist in patient-practitioner communication, promoting trust in clinical practice and benefit clinicians' in exposing considerations that may not have previously been discussed openly. It is also anticipated that the evidence of this Systematic Review will inform healthcare policy makers and support the recent advances and direction towards an evidence based, system wide approach in the development and implementation of open disclosure policy.

\subsection{Review Question}

What are the perceptions and experiences of health professionals to open disclosure of medical errors to patients?

More specifically:

What are the perceptions and experiences of barriers to open disclosure of medical errors to patients by health professionals?

What are the perceptions and experiences of facilitators of open disclosure of medical errors to patients by health professionals? 


\subsection{Inclusion criteria}

\subsubsection{Population of interest}

This review considered studies that included qualified/registered health professionals working in the health system, including those in the position of patient care e.g. doctors, nurses, allied health, as well as managerial and administrative staff. Studies that included student health professionals were excluded.

\subsubsection{Phenomena of Interest}

This review considered studies that investigated health professionals' perceptions and experiences of open disclosure of medical errors to patients.

\subsubsection{Types of studies}

This review considered primary research studies that focused on reporting qualitative data. It was anticipated that the main form of data collection and reporting for this review question would be from individual and group interviews. Literature reviews, opinion papers, discussion papers and other systematic reviews were not considered for inclusion. 


\section{Chapter 2: Methods}

\subsection{Search Strategy}

Prior to the commencement of this research, the JBI Library of Systematic Reviews, the Cochrane Database of Systematic Reviews and the PubMed and CINAHL databases were searched (April - May 2012) to ensure that this specific topic had not been previously addressed by a systematic review to avoid repetition of research of this specific area of interest. No reviews on this topic were located.

The search for this review was conducted using a three-step process. The first step involved a limited search of PubMed and CINAHL using the terms 'error', 'medical errors', 'medical mistake', 'surgical error', 'iatrogenic' 'truth' and 'disclosure'. The results of these initial keyword searches were reviewed for relevant articles. The articles retrieved were reviewed to identify further common keywords and index terms that were used to formulate the comprehensive search strategy subsequently applied across the multiple databases searched (see below) and to clarify and refine concepts and definitions relevant to the topic of interest used in the literature. At this stage assistance from a research librarian was sought to aid in establishing a robust, concise and congruent search strategy to be used in each database (see Appendix 1).

The second step of the search involved a full search of all databases using the identified key words and relevant indexing terms. The search strategy was designed to capture research articles relevant to key concept of the review - the disclosure of medical errors to patients by a variety of health professionals.

The searches were limited to studies in the English language and studies published between 1995 and September 2012. This timeframe was identified to capture the most contemporary information that has been brought to the fore since the release of the 1999 Institute of Medicine report 'To Err is Human: Building a Safer Health System' (Kohn et al, 2000). The search strategy did not include a limitation to the type of 
publication to maximise the sensitivity of the search with the potential to reveal sources for any additional studies. During the review of the citations the following publications types were excluded: commentaries, editorials, systematic reviews, literature reviews, case studies, letters and conference papers.

Two main concepts, 'medical error' and 'truth disclosure', were searched to identify the number of potentially relevant studies for each term. Inclusion of appropriate Medical Subject Headings (MeSH - PubMed, CINAHL and PsycINFO) and EMTREE (Embase) terms were used to ensure sensitivity of the search strategy.

The search terms and concepts were used across the following major medical databases.

PubMed searched 10/09/2012

CINAHL: Cumulative Index to Nursing \& Allied Health Literature (EBSCO Host), searched 15/09/2012

PsycINFO (EBSCOhost), searched 15/09/2012

Embase searched, 10/09/2012

The search for unpublished studies was conducted using the same two (2) concepts, but using simplified versions of keywords such as "medical errors" OR "medical incidents" OR "healthcare errors" OR "treatment errors" AND "truth" OR "disclosure". Searches conducted are listed in Table 2.1.

Table 2.1: Search for Unpublished Studies and date searched

\begin{tabular}{|l|c|c|}
\hline Name & Date searched & Number \\
\hline Mednar & $16 / 09 / 2012$ & 83 \\
\hline WorldWideScience.org & $16 / 09 / 2012$ & 85 \\
\hline $\begin{array}{l}\text { ProQuest Dissertations \& Thesis Databases } \\
\text { (PQDT) }\end{array}$ & $17 / 09 / 2012$ & 12 \\
\hline Total & & 180 \\
\hline
\end{tabular}

Organisational webpages with a focus on patient safety and quality in health care were accessed and are listed in Table 2.2 
Table 2.2: Organisational webpages and date searched

\begin{tabular}{|l|c|c|}
\hline Organisation & Date searched & Number \\
\hline $\begin{array}{l}\text { Australian Commission on Safety and Quality } \\
\text { in Health Care (ACSQHC) }\end{array}$ & $18 / 09 / 2012$ & 3 \\
\hline $\begin{array}{l}\text { Australian Institute for Health and Welfare } \\
\text { (AIHW) }\end{array}$ & $18 / 09 / 2012$ & 3 \\
\hline Institute of Healthcare Improvement (IHI) & $18 / 09 / 2012$ & 24 \\
\hline $\begin{array}{l}\text { Agency of Healthcare Research and Quality } \\
\text { (AHRQ) }\end{array}$ & $18 / 09 / 2012$ & 2 \\
\hline $\begin{array}{l}\text { Joint Commission on Accreditation of } \\
\text { Healthcare Organisation (JCAHO) }\end{array}$ & $19 / 09 / 2012$ & 1 \\
\hline $\begin{array}{l}\text { Joint Commission International Accreditation } \\
\text { (JCIA) }\end{array}$ & $19 / 09 / 2012$ & 7 \\
\hline World Health Organisation (WHO) & $19 / 09 / 2013$ & 2 \\
\hline Total & & 42 \\
\hline
\end{tabular}

The third phase of the search for relevant information involved reviewing the reference lists of all included papers for other relevant studies that had not been previously identified in the search. No further papers were found (see Fig 3.1; Section $3.1)$.

\subsection{Study Selection}

The completed electronic searching generated a list of citations containing title, author, source and an abstract for the majority of results. To assist in managing this information a database was created using the bibliographic citation management software, Endnote ${ }^{\mathrm{TM}}$. Results of the search were exported into the database and an automated scan was initially conducted to identify and remove any duplicate citations. All remaining citations were subsequently assessed by manual screening of the title and abstract (or the title only if an abstract was not available) for relevance and suitability to the inclusion criteria (see Section 1.7). In cases where it was not immediately clear if the paper was eligible to be included in the review based on the information provided, the complete article was retrieved for full text review of relevance to the inclusion criteria of this review. 
Whilst screening titles and abstracts, papers were identified that addressed the disclosure of medical errors and appeared directly relevant to the predefined inclusion criteria; after reviewing the full text however, it became apparent that some of these papers did not involve the disclosure of errors to patients by health profes sionals but rather the acknowledgment and disclosure of errors within a healthcare organisation, including disclosing of errors to colleagues. During the study selection process, the mixed methodology study comprising of a literature review and subsequent focus groups by Kaldjian et al., 2006a relevant to the topic was identified. This study included qualitative data from interviews with medical students as well as qualified health professionals; medical students were not participants of interest for this review. Prior to the final decision being made for inclusion, the author (Kaldjian et al., 2006a) was contacted to gain additional detailed information on the source of the qualitative findings from the focus groups to clarify that this study was eligible for inclusion. There was a prompt response from the author who provided the additional information required to include this study for critical appraisal.

\subsection{Critical Appraisal}

Critical appraisal of the nine (9) identified qualitative papers (see Figure 3.1; Section 3.1) was performed by two independent reviewers to assess the methodological quality of each paper prior to data extraction. The process of critical appraisal was conducted using the internationally recognised and validated standardised critical appraisal instruments from the JBI Qualitative Assessment and Review Instrument (JBI-QARI) (see Appendix II).

During the critical appraisal process it was identified that the results from a significant piece of research involving the implementation of a national Open Disclosure Pilot study across Australia had been reported in various published papers, including Iedema et al., 2007, 2008, 2009 and 2011b. To avoid duplication of data,

the decision was made, to include the first publication of the results of this pilot study that was presented in the form of the Final Report by Iedema et al., 2007 and exclude 
the other three (3) publications that were derived from this final, comprehensive report.

Prior to critical appraisal the two (2) reviewers agreed on a set of guidelines to manage and resolve any potential issues. During this process, it was agreed that the author of the paper would be contacted via email to request any extra information required within two (2) weeks. Another area for consideration was the decision on whether to include or exclude a study. Studies where all ten (10) questions were answered 'yes' by the reviewer would automatically be included in the review. If there was any disagreement between the reviewers' decision of the assessment criteria the disparities would be discussed and debated between the reviewers until an agreement was reached. If an agreement was not able to be reached through discussion between the two (2) reviewers on whether to include or exclude a study, a third reviewer would be asked to review the study.

As part of the critical appraisal process, the corresponding author of Iedema et al., 2007 was contacted to request additional information to gain clarity on the philosophical perspective of the study. There was a prompt response from the author, who provided additional background to the philosophical perspective and the research methodology of the study. With this additional information the study was included in the review.

\subsection{Data Extraction}

Qualitative data was extracted from included papers using the standardised data extraction tool from JBI-QARI (see Appendix III). Relevant detailed information from each study regarding the particular healthcare setting, participants' details and demographics, the country of origin of the research study, the study design and the methods used to collect the data were extracted. In the papers included in this review the study findings were presented and extracted in two (2) main formats; as a table of findings and as a series of identifiable "headings" throughout the research paper. 
Some of the included papers used a single format for reporting the findings, while some of the included papers presented the findings using a combination of a table of findings and headings.

\subsection{Data Synthesis}

Qualitative research findings were pooled using the JBI-QARI program. The process of meta-synthesis was used by aggregating findings to generate a set of statements that represent that aggregation, through assembling the findings rated according to their quality, and categorising these findings on the basis of similarity in meaning. These categories were then subjected to a meta-synthesis in order to produce a single comprehensive set of synthesised findings that can be used as a basis for evidence based practice. 


\section{Chapter 3: Results}

This chapter presents the finding of the systematic review, details the search results, study selection, assessment of methodological quality, and a detailed description of the included studies. The results of the meta-aggregative synthesis are reported by presenting each synthesized finding.

\subsection{Search Results}

The search of bibliographic databases returned a total of 8851 citations. From these results a total of 2945 duplicate citations were identified and removed using both Endnote ${ }^{\mathrm{TM}}$ and manual removal. The remaining papers were screened for eligibility by title and abstract against the inclusion criteria for the review (see Section 1.7), following which 5865 were excluded and 41 papers were retrieved for review of full text. After reviewing these papers, 22 fulfilled the inclusion criteria and were subsequently critically appraised. Following critical appraisal (see Section 2.3), ten (10) studies were excluded on the basis of low methodological quality (see Appendix IV), and nine (9) studies were included in the review for data extraction and synthesis of findings (see Section 3.3).

During the critical appraisal process it was identified that the results from a significant piece of research involving the implementation of a national Open Disclosure Pilot study across Australia had been reported in various published papers, including Iedema et al., 2007, 2008, 2009 and 2011b. To avoid duplication of data, as mentioned in Section 2.3, the decision was made to include the original and detailed publication of the results of this pilot study in the form of the Final Report by Iedema et al., 2007 and exclude the other three publications that were derived from this comprehensive report.

The results of the process are illustrated in Figure 3.1. 
Figure 3.1: Flow diagram detailing results of literature search, study selection and assessment of methodological quality.

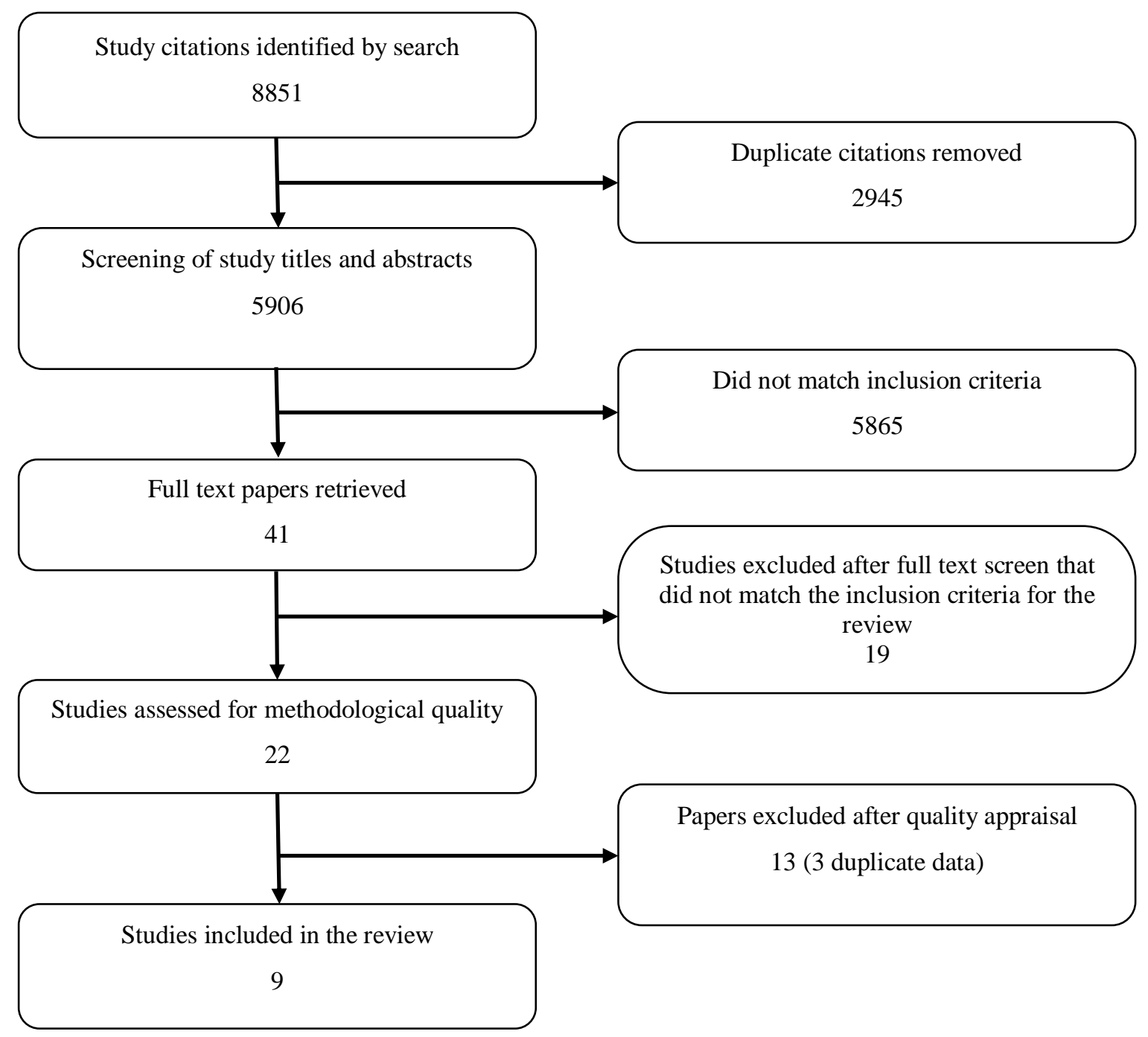

\subsection{Study Selection}

Of the 41 papers retrieved for full text analysis following initial screening of titles and abstracts, a total of 22 studies were deemed to have fulfilled the pre-determined inclusion criteria (Section 1.7). A subset of studies were excluded at this stage as, upon full text review, it was determined that the participants of the studies included students. Several of these studies examined the perceptions of medical students to medical errors and whether patients should be informed about such errors (White et 
al., 2008; Everett et al., 2011). There were also several studies that focused on the educational framework and requirements of students; in particular how they would manage a medical error and the emotional impact it would have on them (Venus et al., 2012). Furthermore, another subset of studies was excluded at this stage; this was because the emphasis of these studies was on the reporting practices of medical errors within an organisation by health professionals. The focus was on the reporting systems that were in place within an organisation and the behaviours of health professionals in using these systems rather than the disclosure of an error to patients. Some studies mentioned the concept of open disclosure of medical errors to patients but on further examination the report of the study did not generate any relevant data in this area.

Studies involving medical errors in the area of pathology and other investigational areas of medicine that appeared to contain qualitative data were also excluded at this stage, as there was a lack of relevant data focused on the disclosure of associated errors to patients. A group of studies that initially appeared to be of a mixed methodology and were considered for extraction of only the qualitative data were also excluded at this time, as further examination of these studies revealed that there was no clear description of how the findings had been obtained. Several other mixed methods studies were excluded, as the focus of the studies was the perceptions of patients, what they expected, and how they would like health professionals to react in the event of medical errors in healthcare. Studies where it was established that the phenomenon of interest was not a central theme of the study were also excluded.

\subsection{Assessment of Methodological Quality}

The quality of the included studies was high. Ten questions were considered with 'Yes', 'No' or 'Unclear' options for each questions. Of the nine (9) included studies, two (2) studies scored 'Yes' 10/10 and two 8/10 (Table 3.1). The remaining five (5) studies scored 'Yes' 7/10 (Table 3.1). Studies scored least well in the area of the congruity between the stated philosophical perspective and the research methods, (the 
philosophical perspective was often not stated), lack of a statement locating the researcher culturally or theoretically, and the influence of the researcher on the research and vice-versa was often not addressed (Table 3.1). Studies with a lack of congruity between the research methodology and the representation and analysis of the data were not included (refer to Appendix IV). Also, those studies where it was not clearly demonstrated and reported how the data gave rise to the specific findings were not included. The results of the critical appraisal process used to establish the methodological quality of the nine (9) selected studies are summarised in Table 3.1. 
Table 3.1: Final Critical Appraisal Assessment of Included Studies

\begin{tabular}{|l|l|l|l|l|l|l|l|l|l|l|}
\hline Citation & Q1 & Q2 & Q3 & Q4 & Q5 & Q6 & Q7 & Q8 & Q9 & Q10 \\
\hline $\begin{array}{l}\text { Fein, S. P., et al., } \\
2007\end{array}$ & $\mathrm{U}$ & $\mathrm{Y}$ & $\mathrm{Y}$ & $\mathrm{Y}$ & $\mathrm{Y}$ & $\mathrm{N}$ & $\mathrm{N}$ & $\mathrm{Y}$ & $\mathrm{Y}$ \\
\hline Greene, D. A., 2009 & $\mathrm{Y}$ & $\mathrm{Y}$ & $\mathrm{Y}$ & $\mathrm{Y}$ & $\mathrm{Y}$ & $\mathrm{Y}$ & $\mathrm{Y}$ & $\mathrm{Y}$ & $\mathrm{Y}$ \\
\hline $\begin{array}{l}\text { De Freitas, G. F., et } \\
\text { al., 2011 }\end{array}$ & $\mathrm{Y}$ & $\mathrm{Y}$ & $\mathrm{Y}$ & $\mathrm{Y}$ & $\mathrm{Y}$ & $\mathrm{Y}$ & $\mathrm{Y}$ & $\mathrm{Y}$ & $\mathrm{Y}$ \\
\hline $\begin{array}{l}\text { De Cassia Pires Coli, } \\
\text { R., et al., 2010 }\end{array}$ & $\mathrm{U}$ & $\mathrm{Y}$ & $\mathrm{Y}$ & $\mathrm{Y}$ & $\mathrm{Y}$ & $\mathrm{U}$ & $\mathrm{N}$ & $\mathrm{Y}$ & $\mathrm{Y}$ \\
\hline $\begin{array}{l}\text { Fein, S. P., et al., } \\
\text { 2005 }\end{array}$ & $\mathrm{N}$ & $\mathrm{Y}$ & $\mathrm{Y}$ & $\mathrm{Y}$ & $\mathrm{Y}$ & $\mathrm{N}$ & $\mathrm{N}$ & $\mathrm{Y}$ & $\mathrm{Y}$ \\
\hline $\begin{array}{l}\text { Gallagher, T. H., et } \\
\text { al., 2003 }\end{array}$ & $\mathrm{U}$ & $\mathrm{Y}$ & $\mathrm{Y}$ & $\mathrm{Y}$ & $\mathrm{Y}$ & $\mathrm{Y}$ & $\mathrm{N}$ & $\mathrm{Y}$ & $\mathrm{Y}$ \\
\hline $\begin{array}{l}\text { Kaldjian, L. C., et al., } \\
2006 a\end{array}$ & $\mathrm{U}$ & $\mathrm{Y}$ & $\mathrm{Y}$ & $\mathrm{Y}$ & $\mathrm{Y}$ & $\mathrm{N}$ & $\mathrm{Y}$ & $\mathrm{Y}$ & $\mathrm{Y}$ \\
\hline $\begin{array}{l}\text { Espin, S., et al., 2006 } \\
\text { Iedema, R., et al., }\end{array}$ & $\mathrm{U}$ & $\mathrm{Y}$ & $\mathrm{Y}$ & $\mathrm{Y}$ & $\mathrm{N}$ & $\mathrm{N}$ & $\mathrm{Y}$ & $\mathrm{Y}$ & $\mathrm{Y}$ \\
\hline $\begin{array}{l}\text { 2007 } \\
\text { \% }\end{array}$ & 22.22 & 88.89 & 100.00 & 100.00 & 100.00 & 44.44 & 33.33 & 100.00 & 100.00 \\
\hline
\end{tabular}

Y= Yes, N=No, U=Unclear (refer to Appendix II for question details) 


\subsection{Description of Included Studies}

Characteristics of included studies are detailed in Table 3.2. The geographical setting of the studies included the United States of America, Canada, Brazil, and Australia. All studies had health care professionals as the participants', some studies included both health professionals and patients - for example, Kaldjian et al., 2006a involved resident physicians from internal medicine; family medicine, paediatrics, community and university based attending physicians. The studies by Gallagher et al., 2003 involved physicians, both academic and community based, as well as patients, Fein et al., 2005 included attending physicians, nurses, hospital administrators and previously hospitalised patients. The Canadian study by Espin et al., 2006 involved operating room team members, nurses, surgeons, anaesthesiologists and a small number of patients who had been discharged post-surgery. The study by Iedema et al., 2007 reported on the Evaluation of the Pilot of the National Open Disclosure Standard in Australia. Participants included doctors, nurses, managerial, support staff, patients, and a small number of family members. Similarly, Fein et al., 2007 included hospital administrators, attending physicians, residents, and nurses. The participants in the study by Greene, 2009 were 17 registered nurses who were responsible for providing nursing care to adult medical and surgical patients in a hospital setting in the USA. Studies by De Freitas et al., 2011 and De Cassia Pires Coli et al., 2010 involved registered nurses with a specialty in Intensive Care Unit (ICU) nursing, practicing in the ICU of the University Hospital and in a large private hospital in the city of Sao Paulo, in Brazil.

Across the studies qualitative data was collected from focus groups, in-depth interviews, and semi-structure interviews. The study by Kaldjian et al., 2006a segregated physicians' into focus groups based on their level of training. The discussion in these groups focused on factors related to physician disclosure of medical errors to institutions, patients and colleagues, the focus groups lasted 90 minutes. Gallagher et al., 2003 conducted focus groups lasting 90 minutes, vignettes of hypothetical situations were presented to participants to generate discussions and 
collect data. The study by Fein et al., 2005, used separate focus groups with open ended questions to probe and reveal the established beliefs, behaviours and perceived barriers and facilitators to disclosure of medical errors to patients. Fein et al., 2007 conducted focus groups and presented participants with a hypothetical case, this generated discussion and details of their own real life experiences of the disclosure of medical errors. Espin et al., 2006 conducted 39 semi structured individual interviews lasting 45 minutes, participants were presented with four (4) hypothetical scenarios and were asked open-ended questions. Iedema et al., 2007, De Cassia Pires Coli et al., 2010 and Greene, 2009 conducted semi structured interviews lasting from 20 minutes up to two (2) hours. De Freitas et al., 2011 collected qualitative data, through in-depth interviews, that lasted between 20-60 minutes. 
Table 3.2: Table of Included Studies Characteristics

\begin{tabular}{|c|c|c|c|c|c|}
\hline Study & Methods & Participants & Intervention & Outcomes & Notes \\
\hline $\begin{array}{l}\text { De Cassa Pires } \\
\text { Coli, R., et al., } \\
2010\end{array}$ & $\begin{array}{l}\text { Semi } \\
\text { structure } \\
\text { interviews, } \\
\text { taped } \\
\text { recorded \& } \\
\text { transcribed } \\
\text { verbatim. } \\
\end{array}$ & $\begin{array}{l}14 \text { nurses working } \\
\text { more than one year } \\
\text { in the Intensive Care } \\
\text { Unit (ICU) who had } \\
\text { experienced a error } \\
\text { during their practice. }\end{array}$ & $\begin{array}{l}\text { The attitudes of } \\
\text { nurses of errors that } \\
\text { occur in nursing } \\
\text { procedures in an } \\
\text { ICU. }\end{array}$ & $\begin{array}{l}\text { This study enables re- } \\
\text { thinking of nursing } \\
\text { practice based on } \\
\text { bioethics, the analysis of } \\
\text { errors \& focusing on the } \\
\text { relationship between } \\
\text { those involved. }\end{array}$ & $\begin{array}{l}\text { Raised the issue of } \\
\text { environmental } \\
\text { impact on how open } \\
\text { disclosure is } \\
\text { managed. }\end{array}$ \\
\hline $\begin{array}{l}\text { De Freitas, G. } \\
\text { F., et al., } 2011\end{array}$ & $\begin{array}{l}\text { In depth } \\
\text { interviews, } \\
\text { audio taped } \\
\text { recorded \& } \\
\text { transcribed } \\
\text { verbatim. }\end{array}$ & $\begin{array}{l}9 \text { Registered Nurses } \\
\text { (RN) with at least } 5 \\
\text { years' experience in } \\
\text { the ICU as a RN \& } \\
\text { experience with } \\
\text { adverse events in the } \\
\text { ICU setting. }\end{array}$ & $\begin{array}{c}\text { Explore the } \\
\text { perceptions \& } \\
\text { attitudes of RNs } \\
\text { towards adverse } \\
\text { events in nursing } \\
\text { care in the ICU } \\
\text { setting. }\end{array}$ & $\begin{array}{l}\text { The various subjective } \\
\text { perspectives about the } \\
\text { number of adverse } \\
\text { events indicate that the } \\
\text { health care system needs } \\
\text { to focus on the } \\
\text { prevention of medical } \\
\text { errors. Ethical behaviour } \\
\text { is essential for patient } \\
\text { safety. }\end{array}$ & $\begin{array}{l}\text { The decision to } \\
\text { disclose an error was } \\
\text { influenced by the } \\
\text { severity of the error. }\end{array}$ \\
\hline $\begin{array}{c}\text { Espin, S., et al., } \\
2006\end{array}$ & $\begin{array}{l}\text { Semi } \\
\text { structured } \\
\text { interviews }\end{array}$ & $\begin{array}{c}9 \text { general surgeons, } \\
9 \text { nurses, } 10 \\
\text { anaesthesiologists \& }\end{array}$ & $\begin{array}{l}\text { Operation room } \\
\text { (OR) team } \\
\text { members' \& }\end{array}$ & $\begin{array}{l}\text { A gap remains between } \\
\text { patients \& team } \\
\text { members' perspective }\end{array}$ & $\begin{array}{l}\text { This study raises } \\
\text { some interesting } \\
\text { issues regarding }\end{array}$ \\
\hline
\end{tabular}




\begin{tabular}{|c|c|c|c|c|c|}
\hline & $\begin{array}{c}\text { using } 4 \\
\text { hypothetical } \\
\text { scenarios } \\
\text { lasting } 45 \\
\text { minutes, \& } \\
\text { were audio } \\
\text { taped. }\end{array}$ & $\begin{array}{l}11 \text { general surgery } \\
\text { patients. }\end{array}$ & $\begin{array}{c}\text { patients' } \\
\text { perceptions of } \\
\text { medical errors, } \\
\text { reporting \& the } \\
\text { disclosure of errors. }\end{array}$ & $\begin{array}{l}\text { on what should be } \\
\text { disclosed \& why. OR } \\
\text { team members use } \\
\text { partial disclosure to } \\
\text { deflect or diffuse } \\
\text { responsibility while } \\
\text { disclosing. The strong } \\
\text { echoes of paternalism } \\
\text { regarding what health } \\
\text { professionals think } \\
\text { should be disclosed to } \\
\text { patients. }\end{array}$ & $\begin{array}{c}\text { disclosure of an } \\
\text { error. }\end{array}$ \\
\hline $\begin{array}{c}\text { Fein, S. P., et } \\
\text { al., } 2007\end{array}$ & $\begin{array}{c}\text { Focus } \\
\text { groups, } \\
\text { audio taped } \\
\& \\
\text { transcribed } \\
\text { verbatim. }\end{array}$ & $\begin{array}{c}55 \text { attending } \\
\text { physicians, } 50 \\
\text { residents, } 45 \text { nurse } \\
\& 54 \text { administrators. }\end{array}$ & $\begin{array}{l}\text { To understand the } \\
\text { discrepancy } \\
\text { between patients' } \\
\text { expectations \& } \\
\text { physicians } \\
\text { behaviours } \\
\text { concerning the } \\
\text { disclosure of errors. }\end{array}$ & $\begin{array}{l}\text { Error disclosure may } \\
\text { mean different things to } \\
\text { clinicians that it does to } \\
\text { patients. We suggest a } \\
\text { definition of open } \\
\text { disclosure to inform } \\
\text { practical policies \& } \\
\text { interventions. }\end{array}$ & $\begin{array}{c}\text { This paper } \\
\text { highlights health } \\
\text { professional hold a } \\
\text { nuanced definition } \\
\text { of open disclosure } \\
\text { creating a potential } \\
\text { communication gap } \\
\text { between clinicians \& } \\
\text { patients in the } \\
\text { aftermath of a } \\
\text { harmful error. }\end{array}$ \\
\hline
\end{tabular}




\begin{tabular}{|c|c|c|c|c|c|}
\hline $\begin{array}{l}\text { Fein, S.P., et } \\
\text { al., } 2005\end{array}$ & $\begin{array}{c}25 \text { focus } \\
\text { groups with } \\
\text { fictional } \\
\text { scenarios, } \\
\text { lasting 60- } \\
90 \text { minutes, } \\
\text { audio taped } \\
\& \\
\text { transcribed. }\end{array}$ & $\begin{array}{c}55 \text { attending } \\
\text { physicians, } 50 \\
\text { residents, } 45 \text { nurses, } \\
54 \text { administrator \& } \\
36 \text { patients. }\end{array}$ & $\begin{array}{l}\text { To explore the } \\
\text { expectations \& } \\
\text { actions of } \\
\text { stakeholders in } \\
\text { order to describe } \\
\text { the factors } \\
\text { influential in the } \\
\text { decision whether to } \\
\text { disclose a medical } \\
\text { error or not. }\end{array}$ & $\begin{array}{l}\text { Significant barriers at } \\
\text { the level of the provider } \\
\& \text { institutional culture } \\
\text { hinder disclosure of } \\
\text { medical errors. }\end{array}$ & $\begin{array}{l}\text { This paper } \\
\text { highlights the } \\
\text { complexity of what } \\
\text { influences health } \\
\text { professional decision } \\
\text { to disclose or not to } \\
\text { disclosure errors to } \\
\text { patients. }\end{array}$ \\
\hline $\begin{array}{l}\text { Gallagher, T. } \\
\text { H., et al., } 2003\end{array}$ & $\begin{array}{c}13 \text { focus } \\
\text { groups } \\
\text { lasting } 90 \\
\text { minutes. } \\
\text { Audiotaped, } \\
\text { transcribed } \\
\text { verbatim \& } \\
\text { reviewed. A } \\
\text { hypothetic } \\
\text { situation } \\
\text { was } \\
\text { presented. }\end{array}$ & $\begin{array}{c}46 \text { physicians \& } 52 \\
\text { patients. }\end{array}$ & $\begin{array}{c}\text { To determine } \\
\text { patients \& } \\
\text { physicians attitudes } \\
\text { about error } \\
\text { disclosure. }\end{array}$ & $\begin{array}{l}\text { Health care institutions } \\
\text { should strengthen } \\
\text { emotional support for } \\
\text { practitioners who have } \\
\text { been involved in } \\
\text { medical errors. }\end{array}$ & $\begin{array}{l}\text { The paper } \\
\text { highlighted the gap } \\
\text { between the } \\
\text { expectations of } \\
\text { patients \& health } \\
\text { professionals to } \\
\text { open disclosure. }\end{array}$ \\
\hline $\begin{array}{l}\text { Greene, D.A., } \\
2009\end{array}$ & $\begin{array}{l}\text { Semi } \\
\text { structured } \\
\text { interviews } \\
\text { audiotaped }\end{array}$ & $\begin{array}{c}17 \mathrm{RN}, 15 \text { female } \& \\
2 \text { male }\end{array}$ & $\begin{array}{l}\text { To gain an } \\
\text { understanding of } \\
\text { the perceptions of } \\
\text { nurses to the }\end{array}$ & $\begin{array}{l}\text { Findings held numerous } \\
\text { implications for nursing } \\
\text { practice \& education. }\end{array}$ & $\begin{array}{l}\text { This thesis raised } \\
\text { interesting issues } \\
\text { regarding open } \\
\text { disclosure, nursing }\end{array}$ \\
\hline
\end{tabular}




\begin{tabular}{|c|c|c|c|c|c|}
\hline & $\begin{array}{l}\text { lasted } 20-60 \\
\text { minutes. }\end{array}$ & & $\begin{array}{l}\text { disclosure of errors } \\
\text { to patients. }\end{array}$ & & $\begin{array}{l}\text { education \& its } \\
\text { impact on nursing } \\
\text { practice. }\end{array}$ \\
\hline $\begin{array}{l}\text { Iedema, R., et } \\
\text { al., } 2007\end{array}$ & $\begin{array}{c}\text { Semi } \\
\text { structured \& } \\
\text { in-depth } \\
\text { interviews } \\
\text { lasting } 45 \\
\text { minutes - } 2 \\
\text { hours, } \\
\text { conducted } \\
\text { in 2007. }\end{array}$ & $\begin{array}{l}131 \text { health care staff, } \\
\text { including } 49 \text { doctors, } \\
20 \text { nurses, } 62 \\
\text { managerial/ support } \\
\text { staff, } 15 \text { patients \& } 8 \\
\text { with family } \\
\text { members. }\end{array}$ & $\begin{array}{c}\text { To evaluate the } \\
\text { implementation of } \\
\text { the National Pilot } \\
\text { of Open Disclosure } \\
\text { Program in } \\
\text { Australia. }\end{array}$ & $\begin{array}{l}\text { There was } \\
\text { overwhelming support } \\
\text { for the Open Disclosure } \\
\text { process; it enhances } \\
\text { relationships with } \\
\text { consumers \& amongst } \\
\text { health care staff. It } \\
\text { presented challenges for } \\
\text { the various stakeholders. }\end{array}$ & $\begin{array}{l}\text { Interesting issues } \\
\text { identified in this } \\
\text { report. Staff felt } \\
\text { relieved to have a } \\
\text { process \& } \\
\text { preplanning } \\
\text { meetings. Active } \\
\text { listening \& } \\
\text { communication } \\
\text { skills are essential } \\
\text { components. The } \\
\text { open disclosure } \\
\text { process is a } \\
\text { challenge \& may } \\
\text { need review. }\end{array}$ \\
\hline $\begin{array}{l}\text { Kaldjian, L., et } \\
\text { al., 2006a }\end{array}$ & $\begin{array}{c}5 \text { focus } \\
\text { groups } \\
\text { lasting } 90 \\
\text { minutes \& } \\
\text { audiotaped, } \\
\text { plus a } \\
\text { literature } \\
\text { review from } \\
\text { a date range }\end{array}$ & $\begin{array}{c}9 \text { attending } \\
\text { physicians, } 9 \\
\text { residents physicians, } \\
\& 7 \text { medical } \\
\text { students. }\end{array}$ & $\begin{array}{l}\text { To develop an } \\
\text { empirically based } \\
\text { comprehensive } \\
\text { taxonomy of factors } \\
\text { that may impede or } \\
\text { facilitate the } \\
\text { voluntary } \\
\text { disclosure of error } \\
\text { by physicians. }\end{array}$ & $\begin{array}{l}\text { A taxonomy of factors } \\
\text { facilitating \& impeding } \\
\text { open disclosure } \\
\text { provided a conceptual } \\
\text { framework for a } \\
\text { complex field of } \\
\text { variables that influence } \\
\text { physicians' willingness } \\
\text { to disclose errors to }\end{array}$ & $\begin{array}{c}\text { This paper } \\
\text { highlighted } \\
\text { impeding factors to } \\
\text { open disclosure that } \\
\text { should be removed } \\
\& \text { facilitating factors } \\
\text { that should be } \\
\text { promoted. }\end{array}$ \\
\hline
\end{tabular}




\begin{tabular}{|l|c|l|l|c|c|}
\hline & of January & & & patients \& colleagues. & \\
$1975-$ & & & & \\
March & & & & \\
\hline
\end{tabular}




\subsection{Meta-aggregative synthesis}

Data extraction from the nine (9) included studies resulted in a total of 131 findings.

The findings were then grouped together based on similarity of meaning and allocated into defined categories. A total of 33 categories were created and were then condensed into a comprehensive set of two (2), final meta-synthesised findings.

The assigned level of credibility for each finding, directly reflecting the congruency of the finding with supporting data from the study from which the finding was taken has been included. The levels of creditability are: Unequivocal [U] relates to evidence beyond reasonable doubt that may include findings that are matter of fact, directly reported/observed and not open to challenge; and Credible [C], which relates to those findings that are, albeit interpretations, plausible in light of the data and theoretical framework (The Joanna Briggs Institute, 2011).

Categories created from the abstracted findings that inform the final synthesis for identified barriers and facilitators of open disclosure can be viewed in Figure 3.2 and Figure 3.3 respectively. To facilitate the narrative description and understanding of the meta-synthesis aligned to barriers (Section 3.5.1) and facilitators (Section 3.5.2) respectively of open disclosure in this section, the narrative describes the meaning and essence of multiple categories in combination or summary that create each synthesised finding. The review revealed a greater amount of findings related to the barriers of open disclosure compared to the facilitators to open disclosure.

\subsubsection{Barriers}

This meta-synthesis of the barriers to open disclosure of medical error to patients by health professionals involved personal fears, professional fears, error factors, patient factors, cultural factors, uncertainties and system factors. It encompasses 19 categories with 74 findings and represents the diversity of identified factors that act as barriers to the open disclosure of medical errors to patients by health professionals. 
The barriers related to personal fears aligned with health professionals avoiding conversations with patients about medical errors to protect themselves from personal discomfort. They feared having to face any potentially negative responses and unpredictable reactions from a patient or their family member when told that there had been an error associated with the patients care. Furthermore, the fear and discomfort of having to admit to making a mistake, a loss in self-confidence in their role to help and not harm people, all acted as barriers to the disclosure of errors. Health professionals also feared losing the trust of their patients and the risk of exposing themselves to liability and litigation.

Professional factors identified as barriers to open disclosure included a lack of confidence in the level of skill to be able to discuss errors with patients. It was also recognised the structured training and education programs for health professionals did not include opportunities to learn about the disclosure of errors to patient or to develop the necessary skills to be prepared for disclosing errors to patients. Furthermore, health professionals were concerned about the negative impact admission of professional error may have on their career and professional reputation. There was also the fear that by disclosing errors to their patients they put themselves at risk of losing any potential career opportunities in the future.

Health professionals often rationalised and provided justification for not disclosing errors to patients. They failed to reveal errors to their patients out of a desire to not only protect the patient but to prevent the patient from experiencing any unnecessary anxiety or stress that may be caused by knowing that there had been a medical error in their care. Similarly, medical errors remain undisclosed when health professionals used selective language and behaviours when communicating with their patients or families. By 'choosing their words carefully' or putting the most 'positive spin' on a situation, health professionals avoid disclosing errors. The evasiveness of the conversation leaves the patient and their family uncertain of what actually happened during their care and were left to connect the dots to reach their own conclusions. 
Error related factors such as the level of harm the patient experienced as a consequence of a medical error also impacted the health professionals decision to disclose an error to the patient or not. If an error did not cause any obvious harm to the patient the health professional was less likely to disclose it to the patient. However, if there were immediate or subsequent consequences for the patient the health professional was more likely to disclose it to the patient. Conversely, if the patient was unaware that a medical error had occurred during the course of their care, the health professional was less likely to disclose it to the patient. Similarly, the health professional's perception of whether the patient would want to know that there had been an error in their care impacted on the decision to disclosure the error to the patient or leave the error undisclosed.

Uncertainties associated with a consistent validated definition of what constitutes an error that was well understood amongst health professionals coupled with a lack of clarity regarding which particular medical errors should be disclosed to patients, were also identified as barriers to open disclosure. Such uncertainties were further compounded by various cultural related factors that acted as barriers to the open disclosure of medical errors to patients.

An organisation with a culture of intolerance to error prevented health professionals from disclosing errors to patients. Cultures of intolerance were characterised by expectations and demands of perfectionism, and prone to quickly place blame on an individual under the assumption that the individuals' poor performance was primarily responsible for the medical error. This cultural phenomenon is a strong deterrent for the acknowledgment of errors and acts as a barrier for the disclosure of medical errors to patients. All these factors contribute and foster a culture of secrecy and silence where it becomes the acceptable norm within an organisation that errors remain hidden from patients. 
Other cultural factors identified as barriers to the disclosure of errors relate to the diversity of the patient and health professional populations. The expanding diversity of the patient population and health professionals brings differences in the expectation, communication style and attitudes towards errors in health care. A lack of understanding, awareness and consideration for individuality and factors that may influence the way they experience health care and medical errors was also identified as a barrier to open disclosure.

System factors, identified as barriers relate particularly to physicians; they felt that disclosing medical errors to patients was an additional demand on their time. They felt that they already practiced in a system under pressure and faced a daily challenge of managing their time and utilisation of available resources to complete an increasingly demanding workload. The disclosure of errors to patients was considered to take too much of their time and required additional resources.

The 19 categories with 74 findings and their accompanying illustrations are presented in Figure 3.2. 
Figure 3.2. Meta aggregative synthesis of the barriers to open disclosure

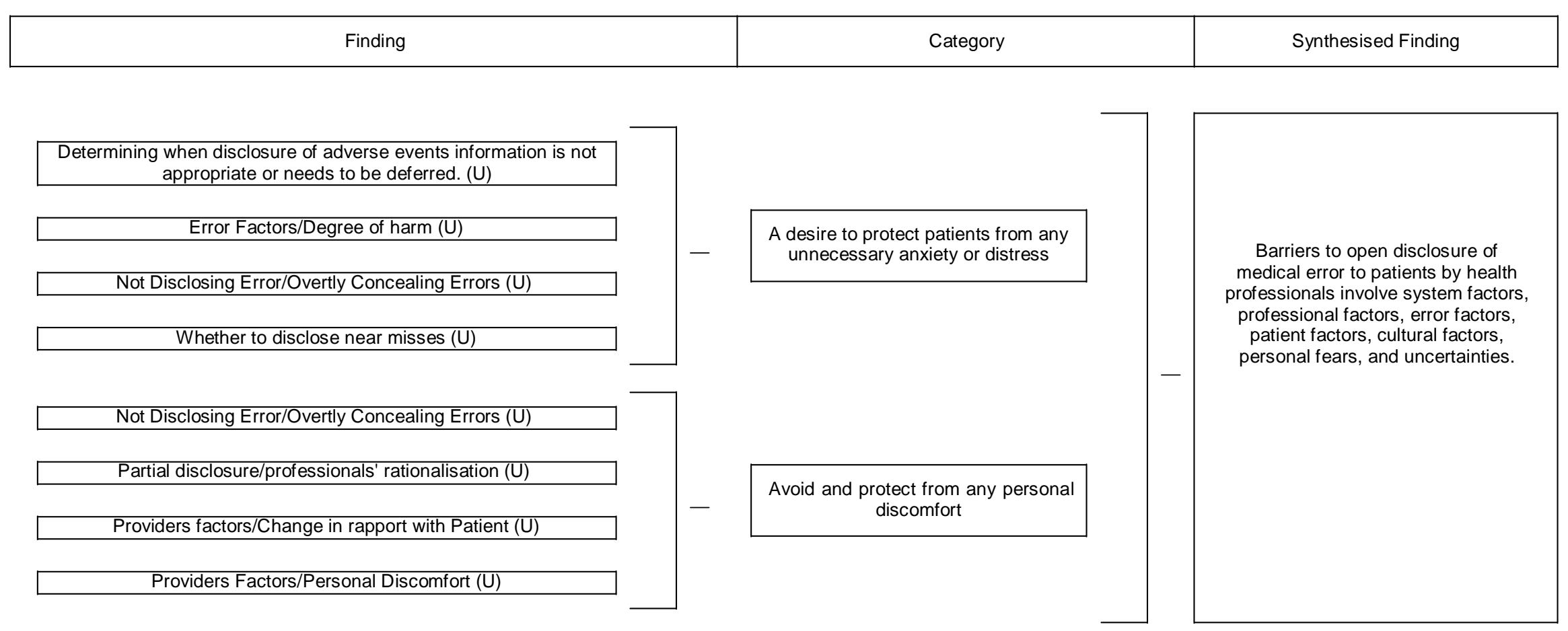




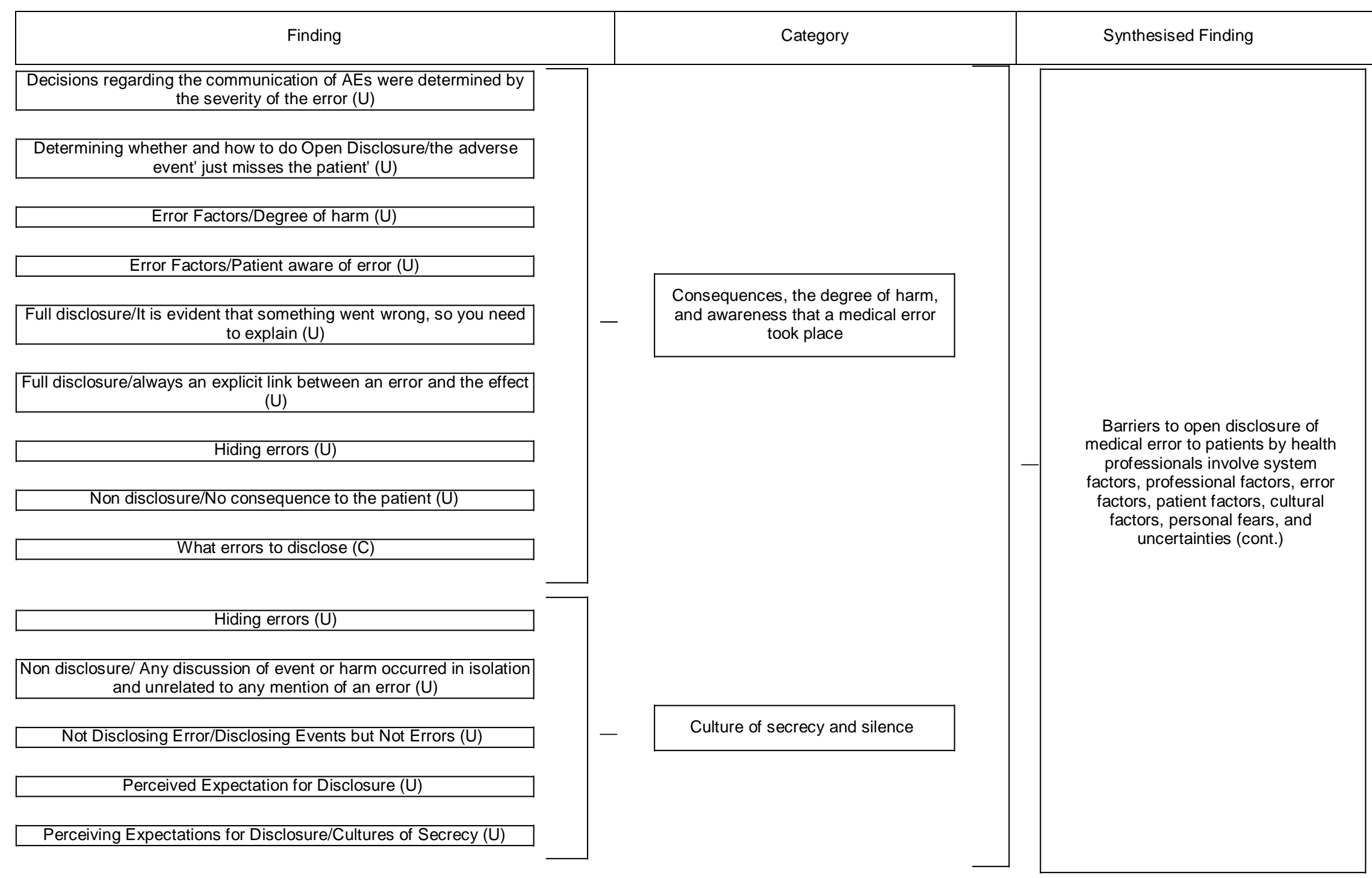




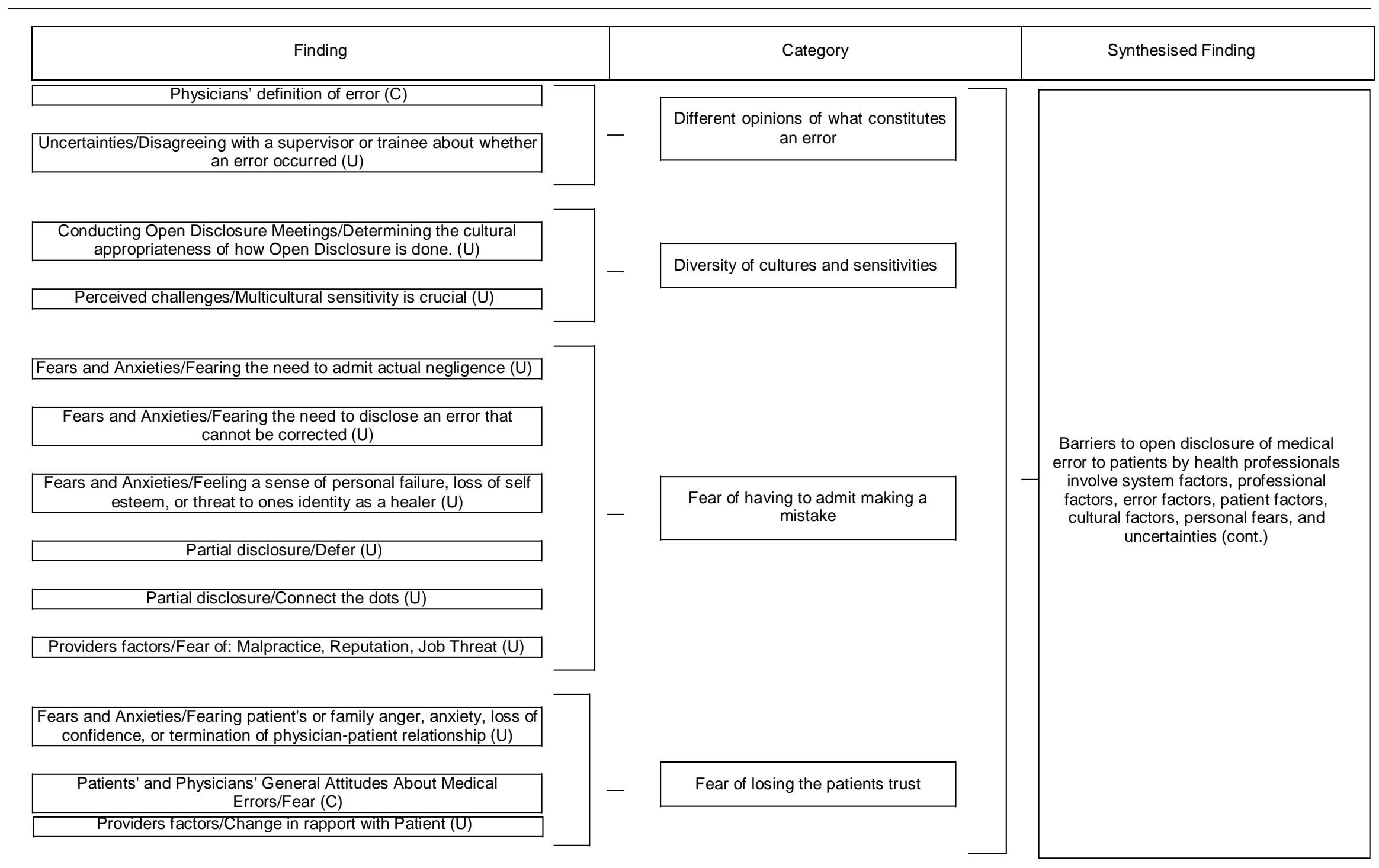




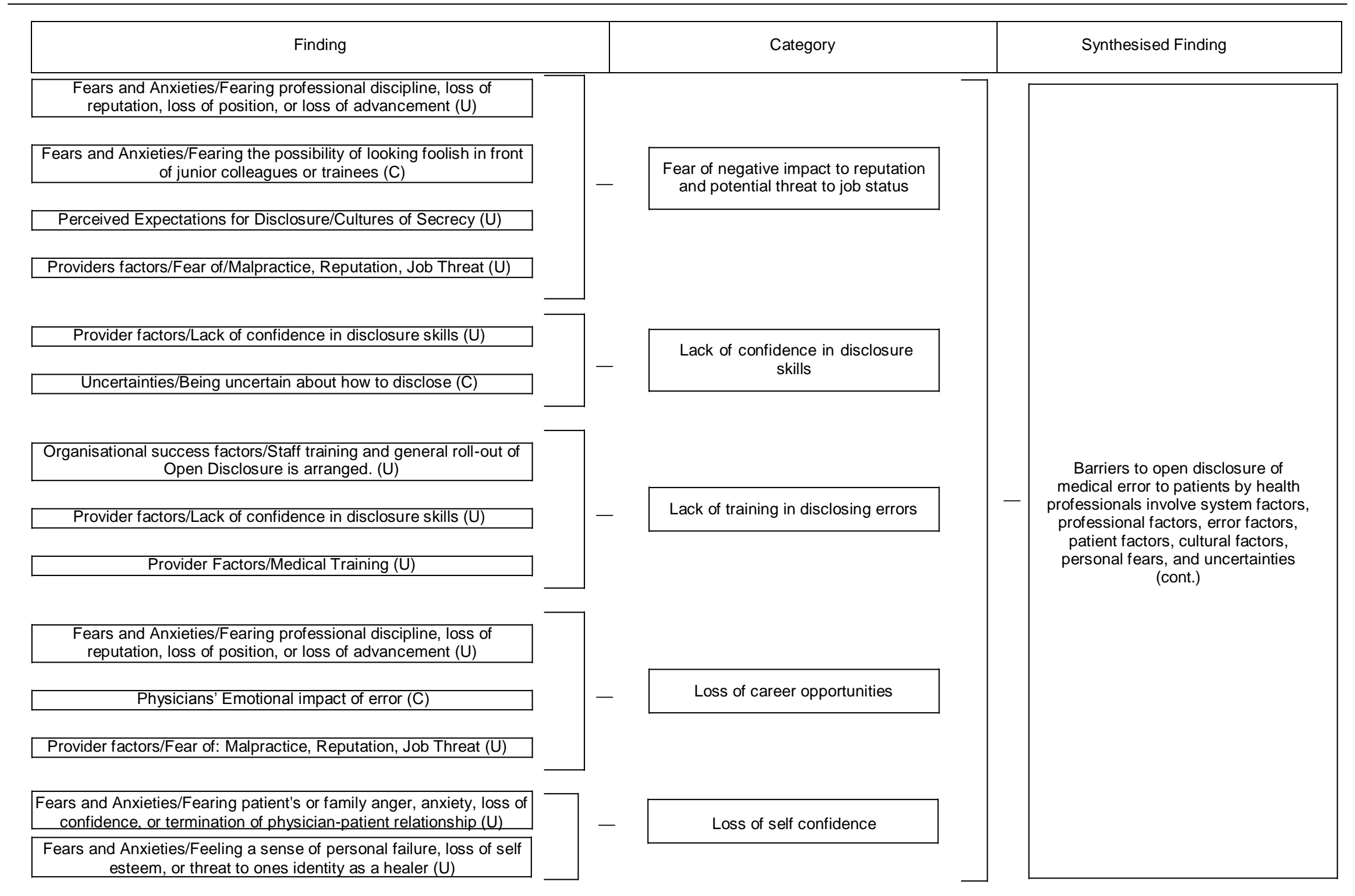




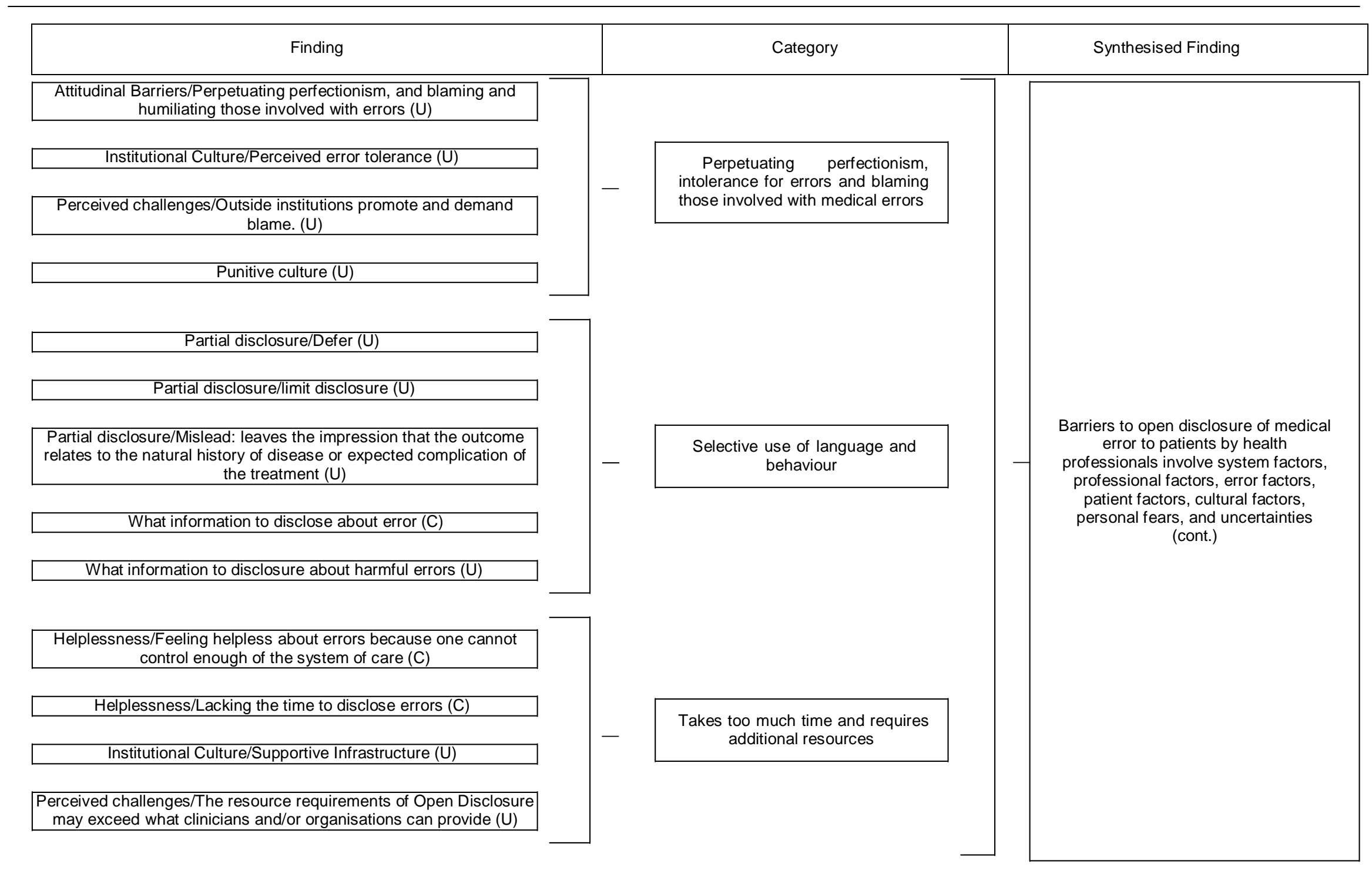




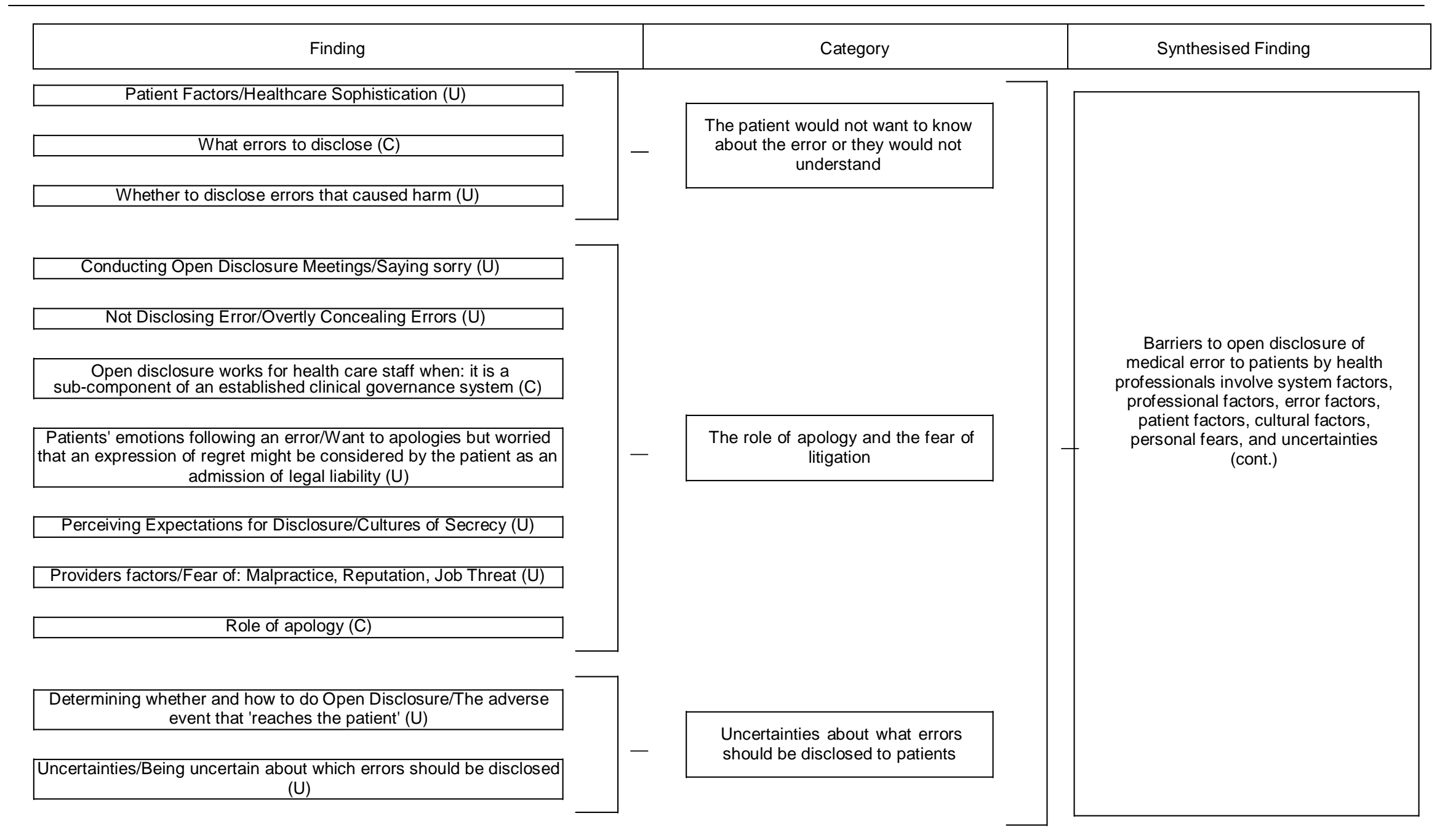




\section{Category 1: Avoid and protect from any personal discomfort}

This category has four (4) findings and represents the fear of an unacceptable level of personal discomfort associated with having conversations with patients about medical error and the fear of having to deal with the patients' reaction.

Finding 1: Not Disclosing Error/Overtly Concealing Errors

Illustration: 'It is difficult to admit your error, because it's embarrassing, and you want to apologize time and time again and you need to have some good answers when they ask the questions and honest answers...Like they could request you not be their nurse anymore. That's their right... and then you feel like they may talk about you.' Greene (2009) pg 93 [U]

Finding 2: Partial disclosure/ professionals' rationalisation

Illustration: professional rationalisations for partial disclosure emphasise factors such as "self protection" the nature of the "patient physician relationship," and a desire not to "create a mess" Espin et al., (2006) pg 9 [U]

Finding 3: Providers Factors/ Change in rapport with Patient

Illustration: "When you go out to talk to the family, [it is] the displeasure they are going to have with you [that matters]. You are going to feel like you are just a bad guy." Fein et al., (2005) pg 489 [U]

Finding 4: Providers Factors/Personal Discomfort

Illustration: "As soon as it gets into the legal realm, suddenly as an attending physician, I feel like I need to be coached as to what can be said and how it can be said and so forth." Fein et al., (2005) pg 490 [U]

\section{Category 2: Fear of having to admit making a mistake}

This category has six (6) findings and represents the fear of having to admit to making a mistake and the sense of shame and failure.

Finding 1: Fears and Anxieties/fearing the need to admit actual negligence

Illustration: 'Saying "I'm sorry' has got to be some of the toughest words in any language and we, as physicians, take a lot of pride in the fact that we're pretty smart and capable people ... To make a mistake that acknowledges my own [fallibility] is in a way saying that I'm not as good as I could be... If it is something like you forgot to 
deflate the catheter that ends in the patient dying, that's a pretty, pretty serious outcome. Like, if you're flying a jet and you drop the bomb on the wrong person. Those things live in your memory forever ...' Kaldjian et al., (2006a) pg 946 [U]

Finding 2: Fears and Anxieties/Fearing the need to disclose an error that cannot be corrected

Illustration: 'Disclosing to the patient makes you admit to yourself that — what's that first tenet of our oath, "First do no harm?"-well, we did harm'. Kaldjian et al., (2006a) pg 946 [U]

Finding 3: Fears and Anxieties/Feeling a sense of personal failure, loss of self esteem, or threat to ones identity as a healer

Illustration: 'I'm delivering bad news to the patient about something, but I'm also delivering bad news about myself because I have been the cause of that bad news.' Kaldjian et al., (2006a) pg 946 [U]

Finding 4: Partial disclosure/Defer

Illustration: MD 1: ' . . being forthright right at the get go and saying, "Look, this happened, we're going to investigate it and we'll get back to you once we have the facts." 'At least in my experience ... has been a more productive way'. MD 2: 'But that's not admitting'. MD 1: 'Right'. Fein et al., (2007) pg 759 [U]

Finding 5: Partial disclosure/Connect the dots

Illustration: "I think you have to be careful, though, that you don't leave the family to have to struggle, or the patient, to connect the dots. The one thing that was missing in your descriptive there was, “And we didn't feed you and we gave you your insulin dosage. And that's what we didn't do" ...” Fein et al., (2007) pg 758 [U]

Finding 6: Providers factors/Fear of: Malpractice. Reputation, Job Threat

Illustration: "I think there are a few kinds of fear. One is fear of what your colleagues are going to think. Two is fear of being sued and what is that going to do to your future, and three is your own internal fear of admitting to yourself that you made a mistake." Fein et al., (2005) pg 489 [U] 


\section{Category 3: Loss of self-confidence}

This category has two (2) findings and represents the loss of confidence associated with errors that inhibits the health professional willingness to disclosure errors to patients.

Finding 1: Fears and Anxieties/Fearing patients or family anger, anxiety, loss of confidence, or termination of physician-patient relationship

Illustration: "[Patients are] coming in here, they're sort of putting their life in my hands, and they're trusting me and I've violated this trust." Kaldjian et al., (2006a) pg 946 [U]

Finding 2: Fears and Anxieties/Feeling a sense of personal failure, loss of self esteem, or threat to ones identity as a healer

Illustration: "I'm delivering bad news to the patient about something, but I'm also delivering bad news about myself because I have been the cause of that bad news.' Kaldjian et al., (2006a) pg 946 [U]

\section{Category 4: Fear of losing the patients trust and rapport}

This category has three (3) findings and represents the underlying fear that if patients are told about errors it will undermine the level of trust they have in the health professionals ability and in their overall treatment plan.

Finding 1: Fear and Anxieties/Fearing patients or family anger, anxiety, loss of confidence, or termination of physician-patient relationship

Illustration: "[Patients are] coming in here, they're sort of putting their life in my hands, and they're trusting me and I've violated this trust." Kaldjian et al., (2006a) pg 946 [U]

Finding 2: Patients and Physicians General Attitudes About Medical Errors/Fear

Illustration: Might harm patients, lawsuits, loss of patient trust, the patient informing friends about their bad experience, loss of colleagues' respect and diminished selfconfidence. Gallagher et al., (2006) pg 1003 [C]

Finding 3: Providers factors/Change in rapport with Patient

Illustration: "loss of rapport with the patient." Fein et al., (2005) pg 489 [U]

Illustration: "trust get[ting] eroded." Fein et al., (2005) pg 489 [U] 


\section{Category 5: Apology and the fear of litigation}

This category has seven (7) findings and represents an overall fear of a link between saying sorry, admitting an error and the risk of liability and litigation.

Finding 1: Conducting Open Disclosure Meetings/Saying sorry

Illustration: "No, you cannot admit liability. You can apologise and say ... we are sorry that this has happened to you, but we cannot turn around and say, yes we can offer you [an explanation] ... and that is some of the anger, because they keep coming back through the course of the meetings and say 'Why don't you not just say that you stuffed up?"' Iedema et al., (2007) pg 50 [U]

Finding 2: Not disclosing Error/Overtly Concealing Errors

Illustration: 'I don't think so. I'll be honest with you in that I don't think so. That was a med error that I found that I corrected. Of course it wasn't up to me. I didn't want to be held liable. I just wanted to document that the corrections were made.' Greene (2009) pg 93 [U]

Illustration: 'I'd rather that nurse who really committed the error fess up to it.' Greene (2009) pg 95 [U]

Finding 3: Open disclosure works for health care staff when it is a sub-component of an established clinical governance system

Illustration: uncertainty about the position taken by insurance and indemnity organisations. Iedema et al., (2007) pg 399 [C]

Finding 4: Patients emotions following an error/want to apologies but worried that an expression of regret might be considered by the patient as an admission of legal liability

Illustration: 'You would love to be just straightforward. "Gosh, I wish I had checked that potassium yesterday. I was busy, I made a mistake, I should have checked that. I can't believe I wouldn't do that. I will learn from my mistake and I will do better next time, because this is how we learn as people." But if you say that to a patient, which you would like to be able to say, honestly, as just another human being, is that we have this whole thing, the wait to cash in [through a lawsuit]'. Gallagher et al., (2003) pg 1005 [U]

Finding 5: Perceiving Expectations for Disclosure/Cultures of Secrecy 
Illustration: "Well that is one part that really confuses me, because I don't know what legally we are allowed to say to patients." Greene (2009) pg 82 [U]

Illustration: 'We as nurse sit around and talk about situations that are bothering us, and I think we all feel pretty much the same way- that we don't have the right to go in and tell the patient what we think happened, because, you know, the legal perspective of it or a threat to what might happen to us if they [administration] find out we told them." Greene (2009) pg 83 [U]

Finding 6: Providers factors/Fear of: Malpractice, Reputation, Job Threat

Illustration: "I think there are a few kinds of fear. One is fear of what your colleagues are going to think. Two is fear of being sued and what is that going to do to your future, and three is your own internal fear of admitting to yourself that you made a mistake." Fein et al., (2005) pg 489 [U]

Illustration: “overwhelmingly, malpractice liability.” Fein et al., (2005) pg 489 [U]

Finding 7: Role of Apology

Illustration: Concerned that apology creates a legal liability. Gallagher et al., (2003) $\operatorname{pg} 1003[\mathrm{C}]$

\section{Category 6: Lack of confidence in disclosure skills.}

This category contains two (2) findings that represent health professionals lack the confidence in their own skill to manage the challenges associated with disclosing medical errors to patients.

Finding 1: Provider factor/Lack of confidence in disclosure skills

Illustration: "My God, this is really uncomfortable and I don't have the confidence about how to do it." Fein et al. (2005) pg 490 [U]

Finding 2: Uncertainties/being uncertain about how to disclose

Illustration: 'focus on doubts about how to disclose errors' Kaldjian et al., (2006a) pg 946 [U] 


\section{Category 7: Lack of training in disclosing errors}

This category has three (3) findings and represents the lack of adequate training and education in developing the skills required to have conversations with patients about medical errors.

Finding 1: Organisational success factors/Staff training and general roll-out of Open Disclosure is arranged.

Illustration: '.. [we need] more staff training in what it means and having more than just the [peer support personnel] relied upon to facilitate it. I think ... having more key people [is important] because the key people, of course, that we trained originally come and go, and some of them have probably left.” Iedema et al., (2007) pg 80 [U]

Finding 2: Provider factors/Lack of confidence in disclosure skills

Illustration: "I learned how to discuss grief and loss.... But error? No... its all on-thejob." Fein et al., (2005) pg 490 [U]

Finding 3: Provider Factors/Medical Training

Illustration: "we might not train our physicians enough about how to go about [disclosing error]." Fein et al., (2005) pg 490 [U]

\section{Category 8: Fear of negative impact to reputation and potential threat to job} $\underline{\text { status }}$

This category has four (4) findings and represents the fear of looking foolish in front of colleagues, loss of respect and the risk of losing their job.

Finding 1: Fears and Anxieties/Fearing professional discipline, loss of reputation, loss of position, or loss of advancement

Illustration: "There's the fear of other people saying, 'Boy, he dropped the ball, he screwed up, he's not really a good doctor, he really doesn't know what he's doing.' You don't want people pointing fingers at you. It's enough to be pointing the finger at yourself, but you don't want other people to say 'he doesn't belong among us." Kaldjian et al., (2006a) pg 946 [U]

Finding 2: Fears and Anxieties/Fearing the possibility of looking foolish in front of junior colleagues or trainees 
Illustration: 'Fear of looking foolish in front of junior colleagues or trainees'. Kaldjian et al., (2006a) pg Appex B [C]

Finding 3: Perceiving Expectation for Disclosure

Illustration: "I think my job would be in jeopardy." Greene (2009) pg 83 [U]

Finding 4: Providers factors/fear of: Malpractice, Reputation, Job Threat

Illustration: "I think fear kind of captures a lot of different emotions that would prevent somebody [from disclosing]...fear of being mistrusted or fear of retribution, fear of damaging career opportunities.” Fein et al., (2005) pg 489 [U]

\section{Category 9: Fear of loss of career opportunities}

This category has three (3) findings and represents the fear associated with the risk of losing future career opportunities.

Finding 1: Fear and Anxieties/Fearing professional discipline, loss of reputation, loss of position, or loss of advancement

Illustration: "There's the fear of other people saying, 'Boy, he dropped the ball, he screwed up, he's not really a good doctor, he really doesn't know what he's doing.' You don't want people pointing fingers at you. It's enough to be pointing the finger at yourself, but you don't want other people to say 'he doesn't belong among us." Kaldjian et al., (2006a) pg 946 [U]

Finding 2: Physicians emotional impact of error

Illustration: Upset that patient was harmed and about how error could impact career Gallagher et al., (2003) pg 1003 [C]

Finding 3: Providers factors/fear of Malpractice, Reputation, Job Threat

Illustration: "I think fear kind of captures a lot of different emotions that would prevent somebody from disclosing.... fear of retribution, fear of damaging career opportunities." Fein et al., (2005) pg 489 [U]

Illustration: "I think there are a few kinds of fear.... what your colleagues are going to think... fear of being sued and what is that going to do to your future." Fein et al., (2005) pg 489 [U] 
Category 10: A desire to protect patients from any unnecessary anxiety or distress

This category has four (4) findings highlighting that health professionals do not tell the patient about a medical error because they wish to protect the patient from any unnecessary anxiety or distress that revealing the error may cause them.

Finding 1: Determining when disclosure of adverse events information is not appropriate, or needs to be deferred.

Illustration: “... this is one case where we actually didn't disclose ... Now, the reason being was that it involved a teenager that was really struggling with his disease ... who was actually quite seriously ill. To say that something went wrong in his particular care would have actually been a detriment to him. The clinician had a very, very good case not to disclose to this particular person, because it would have meant that the work that they'd done beforehand would have been undone because of the frame of mind that he was in at the time. ... It was quite a complex one, but to actually disclose what had went wrong initially was really going to affect his chronic long term management. So that's one where we chose that it was actually better not to. And I think there's got to be room for that.” Iedema et al., (2007) pg 64 [U]

Illustration: "the issue of whether we're doing more harm than good emotionally in raising issues has been raised, so I think it would depend on what the near miss was. ... you still have to argue whether having an Open Disclosure process, that might make the health service feel good but whether it may actually do more harm than good." Iedema et al., (2007) pg 63 [U]

Finding 2: Error Factors/Degree of harm

Illustration: "nothing happened, so there's no reason to bring up an issue that hasn't been brought up and the patient won't bring up" and "for something that didn't cause harm, no need for [the patient] to worry about it." Fein et al., (2005) pg 491 [U]

Finding 3: Not Disclosing Error/Overtly Concealing Errors

Illustration: "I'm sure that [disclosure] would just increase their anxiety level of being in the hospital. Here you have a nurse that has told you she has made a mistake and now, you know, the rest of their [patient's] hospitalization they're going to be in 
there, having an anxiety attack, because they're afraid another nurse might do something to them, to harm them". Greene (2009) pg 92 [U]

Finding 4: Whether to disclose near misses

Illustration: 'My job is to relieve anxiety, not to create it. And to a certain extent when an error occurs that doesn't get to the patient, its not their problem, it's my problem'. Gallagher et al., (2003) pg 1004 [U]

\section{Category 11: Selective use of language and behaviour}

This category has five (5) findings and represents how errors are not disclosed during conversations with patients. Health professionals choose their words carefully; they script a response with the intent of putting the most positive spin on the situation, but not admitting or acknowledging that an error took place.

Finding 1: Partial disclosure/ Defer: suggested other possibilities for an outcome when the source of the error was known, and indicating that further investigation was needed

Illustration: 'It would probably be more reasonable to say, "They fell out of bed and broke their hip - that we know for sure. And we're going to determine why they seized. There are any number of reasons why they seize and we're investigating it. And hopefully we can prevent a similar occurrence from happening in the future." It seems obvious that it was due to low blood sugar, but it may be or it may not be. They may have a seizure disorder to begin with, who knows?' Fein et al., (2007) pg 759 [U]

Finding 2: Partial disclosure/limit disclosure

Illustration: "just the objective truth" Espin et al. (2006) pg 9[U]

Illustration: "The patient's gonna be told... but what you say about how that injury occurred depends..." Espin et al., (2006) pg 9 [U]

Finding 3: Partial disclosure/Mislead: leaves the impression that the outcome relates to the natural history of disease or expected complication of the treatment

Illustration: "Yeah, we didn't get your Coumadin dose quite right, you're going to have to stay in the hospital a couple more days." "does that sound like an error? It 
sounds like we're titrating your medications, when the real story is, I didn't write for it for the last two days, thats why'. Fein et al., (2007) pg 759 [U]

Illustration: "...... You tell the truth. But it's a contextual truth”. Fein et al., (2007) pg $759[\mathrm{U}]$

Illustration: "..should it be framed in the context of a mistake as opposed to saying that diabetics have wide swings in their blood sugar and it was just wider than expected and he had an injury because of that?" Fein et al., (2007) pg 759 [U]

Finding 4: What information to disclose about error

Illustration: "choose words carefully." Gallagher et al., (2003) pg 1003 [U]

Finding 5: What information to disclose about harmful errors?

Illustration: "I think you have to be a spin doctor all the time and put the right spin on it... I don't think you have to soft pedal this issues, but I think you have to try to put it in the best light”. Gallagher et al., (2003) pg 1004 [U]

Illustration: 'I would be very straightforward and say "You were given too much insulin. Your blood sugar was lowered and that's how you arrived in the intensive care unit. You were given some dextrose . . . " and apologize for the events. And then if they want to know . . . "How did I get too much, or why couldn't they read your writing, or why didn't they call you?" you go into those individually, but I wouldn't walk in saying I have sloppy handwriting and they didn't know what they were reading. You just tell the facts: "You got a big bunch of insulin and your blood sugar went down, and we got that fixed up and we're glad you're great."' Gallagher et al. (2003) pg 1004 [U]

Illustration: 'I would say something like "I thought this medication was appropriate for you. I didn't anticipate it to have this response." And I don't disclose the fact that I didn't check the lab that I was supposed to check'. Gallagher et al., (2003) pg 1005 [U]

Illustration: 'My approach to this would be to say "I ordered the medicine for you, one of the effects of which is your potassium went up. I ordered the laboratory tests. I didn't recognize that it was getting high until the second test. And its high, you are having arrhythmias, and we are treating you. "I don't know that I would say "I 
ordered the test. I tried to find the result. I didn't get the result. I forgot about it, and I never checked it." I wouldn't say that' Gallagher et al., (2003).pg 1005[U]

Category 12: Consequences, the degree of harm, and awareness that a medical error took place

This category has nine (9) findings and represents non-disclosure of an error in circumstances where there has been no immediate consequence for the patient and where there has been no harm to the patient and the patient is unaware that an error took place.

Finding 1: Decisions regarding the communication of adverse events were determined by the severity of the error.

Illustration: "I evaluate the impact of an error on the patient. The patient and his family are told about the adverse events only when there is the possibility of harm". De Freitas et al., (2001) pg 336 [U]

Finding 2: Determining whether and how to do Open Disclosure/ the adverse event just misses the patient

Illustration: "Near misses, the way we describe near misses is that there was a potential for an error to occur but it hasn't reached the patient, so therefore we don't [disclose], because it has not happened. You know, somebody stopped it from happening.” Iedema et al., (2007) pg 31 [U]

Illustration: "You don't need to go and necessarily tell a patient", 'Oh, we had a near miss with you'. Iedema et al., (2007) pg 33 [U]

Finding 3: Error Factors/Degree of harm

Illustration: "nothing happened, so there's no reason to bring up an issue that hasn't been brought up and the patient won't bring up" and "for something that didn't cause harm, no need for [the patient] to worry about it." Fein et al., (2005) pg 491 [U]

Finding 4: Error Factors/Patient aware of error

Illustration: "errors that are either evident to a patient or have immediate consequences or significant consequences to the patient.” Fein et al., (2005) pg 491 [U] 
Finding 5: Full disclosure/It is evident that something went wrong, so you need to explain

Illustration: "You had to convert to open, so you need to explain that" Espin et al., (2006) pg 10[U]

Finding 6: Full disclosure: always an explicit link between an error and the effect

Illustration: 'Your hip broke. The reason for that was that your sugar was so low and the reason that your sugar fell low was because you did not have any food and the medication was not withheld. It was an error and unfortunately you had a bad outcome.' Fein et al., (2007) pg 757 [U]

\section{Finding 7: Hiding errors}

Illustration: "And sometimes, when we do something wrong, we don't report either. It stops right there, unless it is something really serious... Otherwise you leave it behind the scenes and... I think this happens a lot.” De Freitas et al., (2011) pg 329 [U]

Illustration: "I guess that, like it's interesting: people hide a mistake when they know that it won't have immediate consequences for the patient[...] Now if someone makes a mistake and knows nobody saw it, nobody realized it, I guess people omit reporting it.” De Cassia Pires Coli, et al., (2010) pg 329 [U]

Finding 8: Non disclosure/No consequences to the patient

Illustration: "You still have to enlarge the incision, so there would be no consequence whatsoever for the patient" Espin et al., (2006) pg.10 [U]

Finding 9: What errors to disclose?

Illustration: 'errors that harm, except when harm is trivial, patient cannot understand error, or patient does not want to know about error.' Gallagher et al., (2003) pg 1003 [U]

Category 13: The patient would not want to know about the error or they would not understand

This category has three (3) findings and represents the decision not to disclose errors based on the belief that the patient would not want to know.

Finding 1: Patient Factors/healthcare sophistication 
Illustration: "I almost think it needs to be on a patient-by patient basis. The same case could happen to two different patients. And it may be appropriate to report one, whereas it may not be so appropriate to report the other, depending upon the patient's overall psyche.” Fein et al., (2005) pg 490 [U]

Finding 2: What errors to disclose?

Illustration: errors that harm, except when harm is trivial, patient cannot understand error, or patient does not want to know about error. Gallagher et al., (2003) pg 1003 $[\mathrm{C}]$

Finding 3: Whether to disclose errors that caused harm?

Illustration: 'You don't want to be accused of scaring people. I've had patients tell people that I was scaring them when I thought I was simply being informative and, you know not being dramatic or anything. But clearly in those cases I was telling people more that they wanted to know'. Gallagher et al., (2003) pg 1004 [U]

\section{Category 14: Different opinions of what constitutes an error}

This category has two (2) findings and represents a lack of consistency a definition amongst health professional of what is considered to be a medical error.

Finding 1: Physicians definition of error

Illustration: Narrow: deviations from accepted standards of care only. Gallagher et al., (2003) pg 1003 [C]

Finding 2: Uncertainties/Disagreeing with a supervisor or trainee about whether an error occurred

Illustration: "Medicine is vague enough sometimes that even though I feel [an error occurred] there's no higher power for me to appeal to if the higher power within my group feels that the right thing was done." Kaldjian et al., (2006a) pg 946 [U]

\section{Category 15: Uncertainties about what errors should be disclosure to patients}

This category has two (2) findings and represents experiences of uncertainties about errors and whether it is considered necessary to tell the patient about all medical error/event that may have occurred during the course of their care. 
Finding 1: Determining whether and how to do Open Disclosure/the adverse event that 'reaches the patient'

Illustration: "Perhaps we need more clarity about severity of things that need disclosing. There are clearly very minor things. I reckon Open Disclosure about minor things causes havoc without benefit that things must be disclosed. So, guidance about what do disclose and what not to disclose in a more corporate way might be helpful.” Iedema et al., (2007) pg 29 [U]

Illustration: "There is a difference between complication of treatment and stuff-ups of treatment. We have to be open about complications and stuff-ups." Iedema et al., (2007) pg 29 [U]

Finding 2: Uncertainties/Being uncertain about which errors should be disclosed Illustration: "At what level does the error become big enough that now something needs to be done about it?" Kaldjian et al., (2006a) pg 946 [U]

\section{Category 16: Diversity of cultures and sensitivities}

This category has two (2) findings and represents the impact diverse cultural backgrounds have on open disclosure of medical error.

Finding 1: Conducting Open Disclosure Meetings: Determining the cultural appropriateness of how Open Disclosure is done

Illustration: "[we need] cultural awareness. [For example] a lot of indigenous people will give you eye contact and this older guy was really annoyed at the nurses because they wouldn't give him eye contact [but we] don't understand the various community groups and their nuances." Iedema et al., (2007) pg 56 [U]

Illustration: "[name overseas-trained junior doctor] needed it to be explained to him what was going to happen because it's probably not as common a thing in [his] subcontinent. And most junior clinicians aren't aware of it in the [name State] system." Iedema et al., (2007) pg 56 [U]

Finding 2: Perceived challenges/Multicultural sensitivity is crucial

Illustration: "..some of the different ethnic groups, ... they have different expectations and different values associated with health care." Iedema et al., (2007) pg $84[\mathrm{U}]$ 


\section{Category 17: Culture of secrecy and silence}

This category has five (5) finding and represents the impact organisational culture has on the practice of disclosing medical errors to patients.

Finding 1: Hiding errors

Illustration: "cause everything ends up hidden." De Cassia Pires Coli, et al., (2010) pg 329 [U]

Illustration: "And sometimes, when we do something wrong, we don't report either. It stops right there, unless it is something really serious...Otherwise you leave it behind the scenes and... I think this happens a lot". De Cassia Pires Coli, et al., (2010) pg 329 [U]

Finding 2: Non disclosure: any discussion of event or harm occurred in isolation and unrelated to any mentioned of an error

Illustration: '..it's probably once a month or more often we'll have some old person come in with a massive intracranial fatal hemorrhage, and the INR is 4 or 6 or 10 . We don't tell them.' Fein et al., (2007) pg 758 [U]

Illustration: "There was one case that we had, a guy who got two doses of Lovenox back to back. Nursing error. And he then had a...hemorrhage and went to the unit. Now, I filled out an incident report, but I didn't go running and talking to the patient's family about giving him two Lovenox doses." Fein et al., (2007) pg 758 [U]

Finding 3: Not Disclosing Error/ Disclosing Events but Not Errors

Illustration: [discovered the error so woke the patient in the middle of the night] "its time to start your IV."[the patient was left wondering why]. Greene (2009) pg 89 [U]

Illustration: [describe conversation with patients about errors in which terms such as]"complication" and "adverse effect" [were used to disclose events but not errors] Greene (2009) pg 89 [U]

Illustration: "It was a bolus of potassium, and it went in too fast...it should have been at a slower rate." Greene (2009) pg 89 [U]

Finding 4: Perceiving Expectation for Disclosure 
Illustration: "Telling patients about errors? Oh, no. Oh, no. They [hospital administration] will not disclose that, they would like you to write it down, and it's done discreetly." Greene (2009) pg 77 [U]

Finding 5: Perceiving Expectation for Disclosure/Cultures of Secrecy

Illustration: "I had some strong feelings about it [disclosure]. I thought that the doctor should have told them what happened.” Greene (2009) pg 82 [U]

Illustration: "The culture dictates I shouldn't say anything, just take care of the patient from that point on, for the best outcome that could come out of that." Greene (2009) pg 82 [U]

Illustration: 'We as nurse sit around and talk about situations that are bothering us, and I think we all feel pretty much the same way- that we don't have the right to go in and tell the patient what we think happened, because, you know, the legal perspective of it or a threat to what might happen to us if they [administration] find out we told them." Greene (2009) pg 83 [U]

Illustration: "Well... there's really not a policy, that requires that you tell them that something like that [an error] has happened." Greene (2009) pg 86 [U]

Illustration: "As far as I know that is as far as it went." Greene (2009) pg 87 [U]

\section{Category 18: Perpetuating perfectionism, intolerance for errors and blaming} those involved with medical errors

This category has four (4) findings and represents issues surrounding expectations of perfectionism, intolerance for errors and a punitive culture, which deters and disempowers health professionals from disclosing medical errors to patients.

Finding 1: Attitudinal Barriers/Perpetuating perfectionism, and blaming those involved with errors

Illustration: " Even though I know it's not logical for me to think that doctors aren't going to make errors, I hold doctors to a standards, that we're going to be perfect and we're not going to make errors". Kaldjian et al., (2006a) pg 946 [U]

Finding 2: Institutional Culture/Perceived error tolerance 
Illustration: "The remark - Goddamn, what were you thinking? - comes out pretty often. When you get that kind of response when you admit your error, you are very unlikely to continue admitting your errors." Fein et al., (2005) pg 492

Finding 3: Perceived challenges/Outside institutions promote and demand blame

Illustration: "I think part of the problem there is that, we talk about a no-blame culture or a just culture or whatever you want to call it, but the problem still remains that when something goes wrong it ends up in the public domain.... "Iedema et al., (2007) pg 82 [U]

Finding 4: Punitive culture

Illustration: Mistakes are not allowed! It seems that everything has to work right. How come you can make mistakes in other professions... and in our profession you can't. A mistake can wipe out everything you've done." De Cassia Pires Coli, et al., (2010) pg $329[\mathrm{U}]$

\section{Category 19: Takes too much time and additional resources}

This category has 4 (four) findings and represents feelings of a lack of control over the system, and a lack of time to available to spend disclosing medical errors to patients.

Finding 1: Helplessness/Feeling helpless about errors because one cannot control enough of the system of care. Kaldjian et al. (2006a) pg 946[U]

Illustration: Not having the power necessary to improve the system of care. Kaldjian et al., (2006a) pg 946 [C]

Finding 2: Helplessness/Lack of time to disclose errors

Illustration: Discussing errors with patients requires too much time Kaldjian et al., (2006a) pg apex B [C]

Finding 3: Institutional Culture/Supportive Infrastructure

Illustration: "There needs to be a culture where individuals do not feel penalized for reporting errors. You should feel comfortable reporting to the chief of service or the head of nursing." Fein et al., (2005) pg 492 [U] 
Illustration: “...[S]how people, 'This is how [to be] a good physician,' by attending who acknowledge those kinds of things and discuss them ...." Fein et al., (2005) pg 492 [U]

Finding 4: Perceived challenges/The resources requirements for Open Disclosure may exceed what clinicians and/or organisation's can provide

Illustration: "People don't like to talk about funding with quality. They think quality comes from nothing and it doesn't require any money. It just requires an attitudinal change or something like that, but, unfortunately that's not true in my opinion. Quality costs money and to have a good quality program costs a large amount of money and usually you can't make guarantees to the family that this will be improved because the amount of funding required to improve it is not available. So that's how it improves it, but that's, mm. I mean we had no radiology at this hospital for over a year so, I mean we had a lot of problems with misses and things on scans. So the family go, 'Well, aren't you getting radiologists?' Iedema et al., (2007) pg 83 [U]

\subsubsection{Facilitators}

This meta-synthesis of facilitators to open disclosure of medical errors to patients by health professionals incorporates professional factors, personal factors, error factors, cultural factors and system factors. It encompasses 14 categories with 57 findings. Figure 3.3 represents the diversity of identified factors that act as facilitators to the open disclosure of medical errors to patients by health professionals.

A sense of professional responsibility facilitated the disclosure of medical errors to patients. Health professionals felt more comfortable disclosing errors in care to their patients when they had a good rapport and established relationship with the patient and their family. The decision whether to disclose an error to a patient was also influenced by the motivation to foster and develop positive inter-professional relationships with their colleagues that were based on mutual support and trust. 
Similarly, errors were disclosed to patients because health professionals considered it an opportunity to learn what went wrong and work towards implementing corrective actions to prevent it from happening again. By sharing the lessons learnt from mistakes and demonstrating behaviours of transparency to their patients', health professionals acted as a role model to other staff to engage in the practice of open disclosure. Effective communication and listening skills also facilitated the process of open disclosure. By allowing the patients to express themselves and have their rights and emotions acknowledged, it provided the patient with a sense of control and helped build trust in the health system.

Furthermore, the disclosure of medical errors provided a forum for health professionals to educate patients, their family members and ultimately the wider community of the complexity of healthcare and the associated risks. Similarly, health professionals described feeling a sense of relief with the knowledge that there was a structured process to guide and support them through open disclosure of errors.

The findings of this systematic review identified the personal needs of health professionals acted as facilitators to open disclosure, the desire to be accountable for one's own actions and the need to seek forgiveness from the patient or family members who had been involved in a medical error. Health professionals' experienced the need to alleviate their own personal feelings of guilt after being involved with an error in their patients care, and believed informing the patient was the right thing to do.

Organisational culture factors clearly influenced the practice of open disclosure. Organisations with identified processes and structures to manage medical errors created supportive environments for the practice of open disclosure. Transparency of medical error information and mechanisms to acknowledge and deal with errors helped create a culture of honesty and trust. When health professionals feel supported by the organisation and their colleagues they are more willing to admit to mistakes in care and disclose information to their patients. Furthermore, a system wide approach 
to the identification and implementation of effective supportive frameworks that encompass pre planning of open disclosure meetings and services that support the needs of the staff and patient all acted as facilitators for open disclosure.

The 14 categories with 57 findings are presented in Figure 3.3. 
Figure 3.3. Meta-aggregative synthesis of facilitators of open disclosure

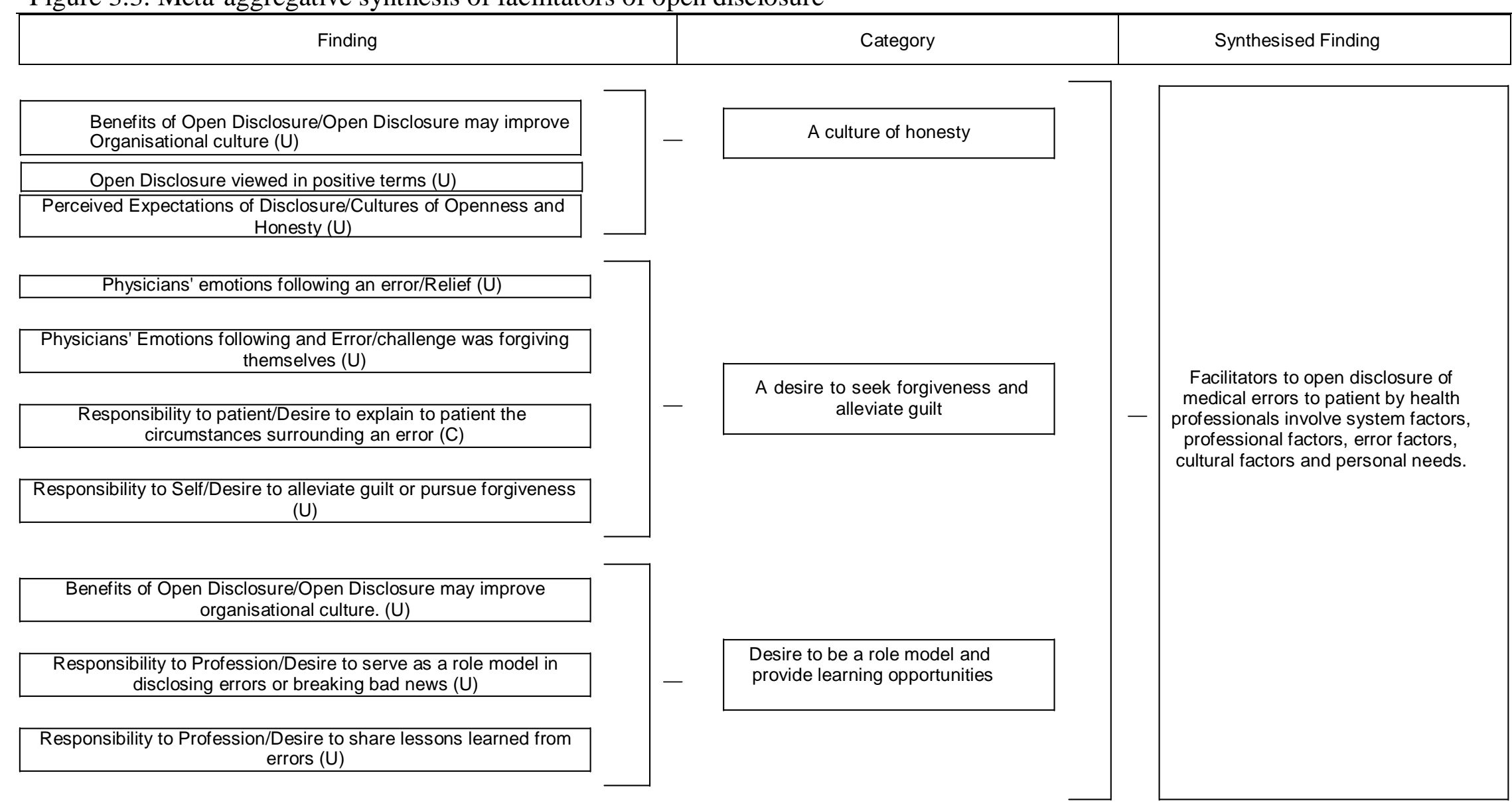




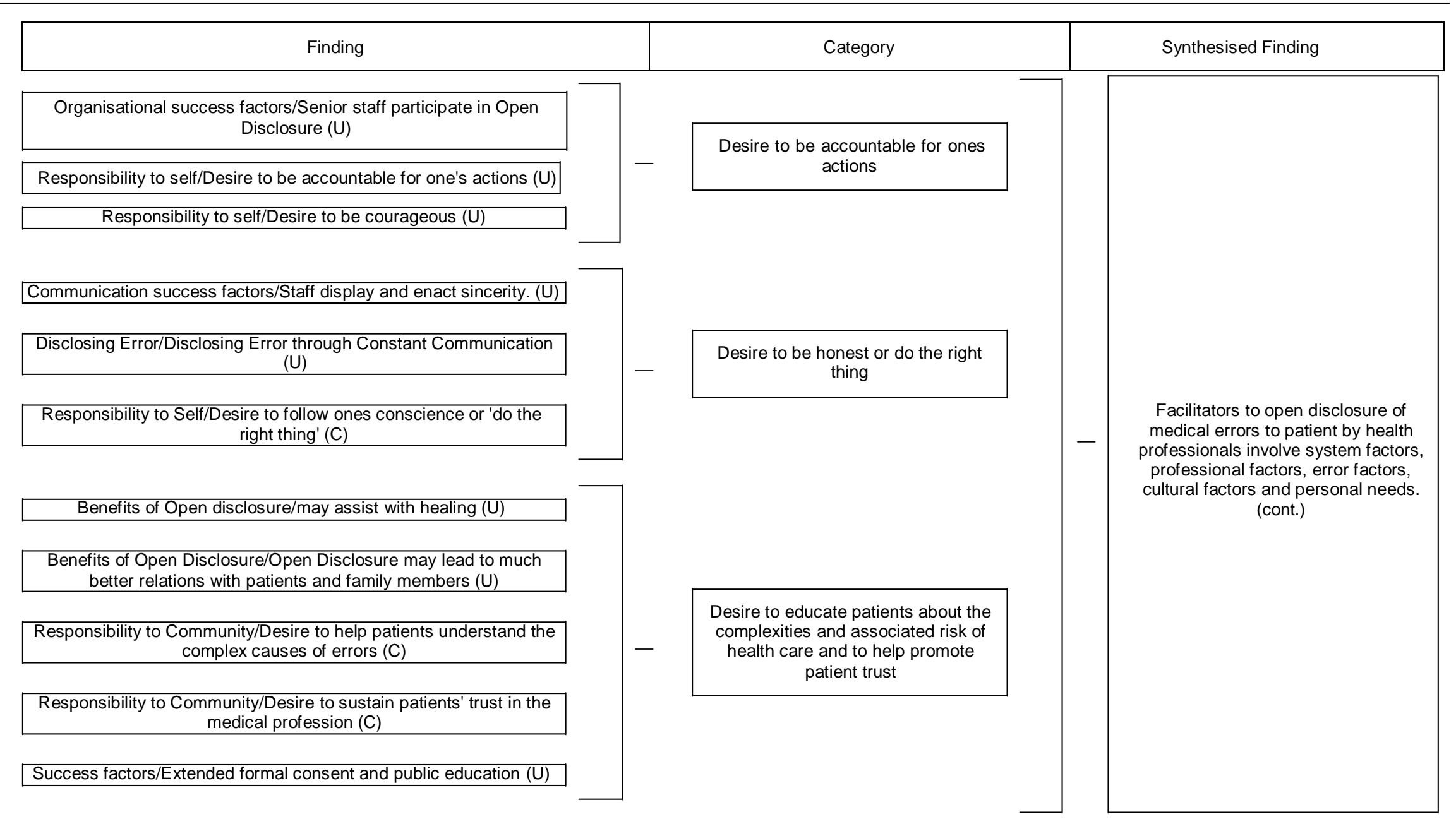




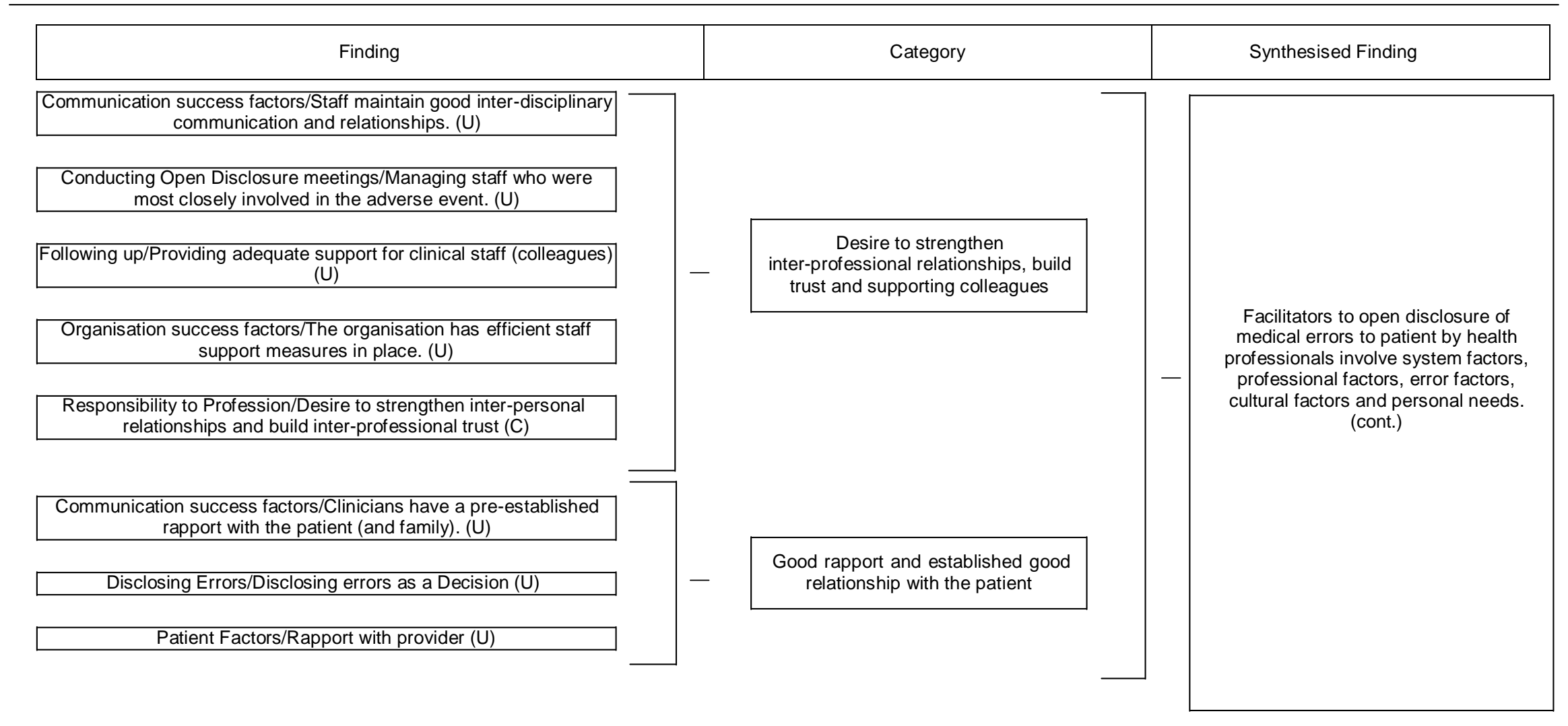




\begin{tabular}{|c|c|c|c|c|}
\hline Finding & & Category & & Synthesised Finding \\
\hline \begin{tabular}{|c|} 
Acknowledging and communicating errors (U) \\
$\begin{array}{c}\text { Conducting Open disclosure/Distinguishing between conventional } \\
\text { ways of dealing with unexpected outcomes and the practices required } \\
\text { by Open Disclosure. (U) }\end{array}$ \\
\end{tabular} & \multirow{3}{*}{-} & \multirow{3}{*}{$\begin{array}{l}\text { Integrated processes, and services } \\
\text { to support staff }\end{array}$} & \multirow{3}{*}{-} & \multirow{3}{*}{$\begin{array}{l}\text { Facilitators to open disclosure of } \\
\text { medical errors to patient by health } \\
\text { professionals involve system factors, } \\
\text { professional factors, error factors, } \\
\text { cultural factors and personal needs. } \\
\text { (cont.) }\end{array}$} \\
\hline $\begin{array}{c}\text { Following up/Creating and maintaining organisational memory. (U) } \\
\text { Institutional Culture/Supportive Infrastructure (U) } \\
\begin{array}{c}\text { Organisational success factors/Organisational-structural prerequisites } \\
\text { have been put in place. (U) }\end{array} \\
\end{array}$ & & & & \\
\hline $\begin{array}{c}\text { Staff are supported in confronting legal, insurance and professional } \\
\text { (reputation) uncertainties (U) }\end{array}$ & & & & \\
\hline
\end{tabular}




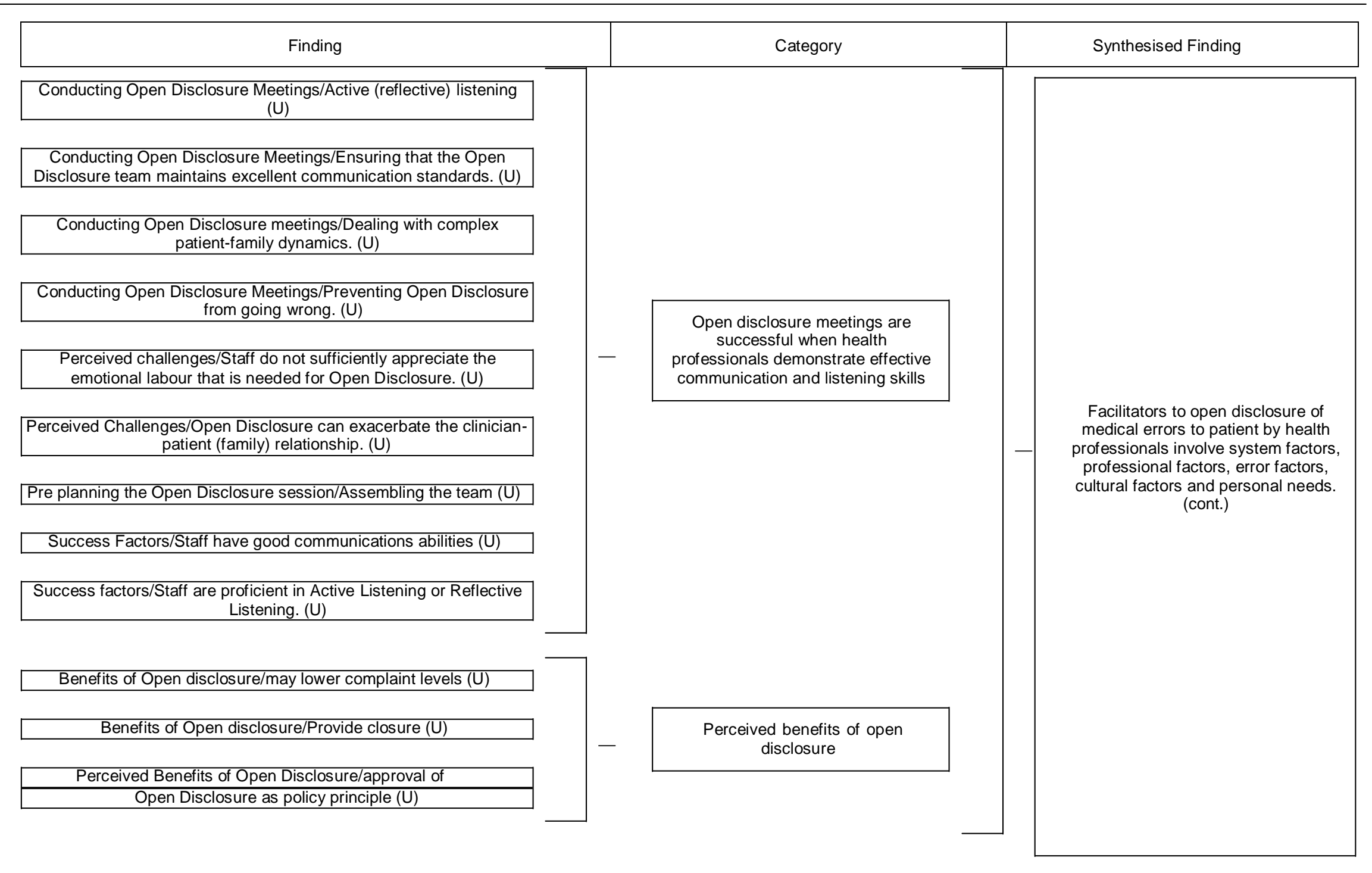




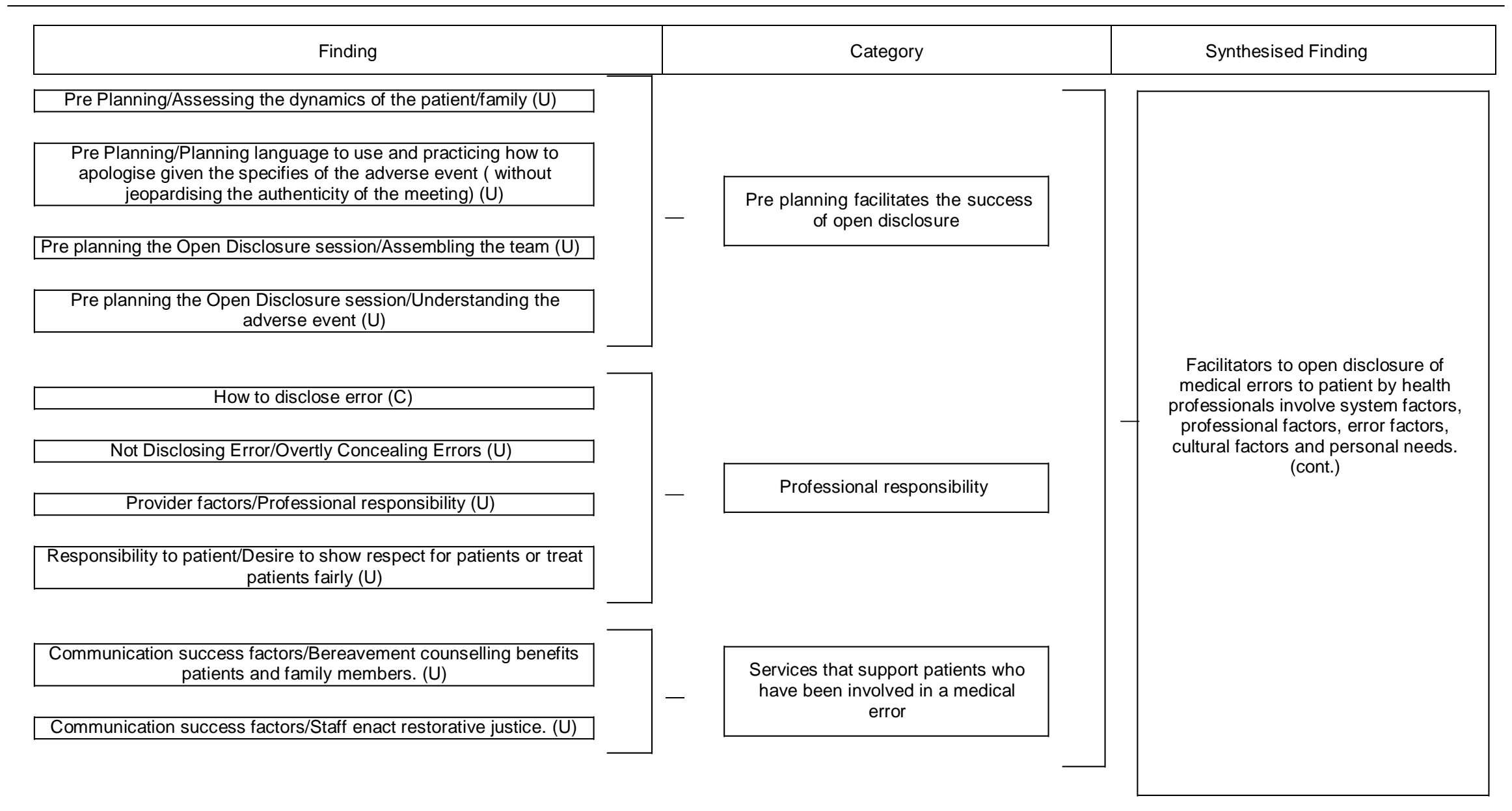




\section{Category 1: Professional responsibility}

This category has four (4) findings and represents professional characteristics identified by study participants of taking responsibility for ones actions.

Finding 1: How to disclose error

Illustration: 'truthfully, objectively professionally'. Gallagher et al., (2003) pg 1003 [C]

Finding 2: Not Disclosing Error/Overtly Concealing Errors

Illustration: 'I'd rather that nurse who really committed the error fess up to it.' Greene (2009) pg 95 [U]

Finding 3: Provider factors/Professional responsibility

Illustration: "No matter what happens in the care of the patient... I am the one who is responsible for that patient." Fein et al., (2005) pg 489 [U]

Illustration: "Its my obligation to do so". Fein et al., (2005) pg 489 [U]

Finding 4: Responsibility to patient/Desire to show respect for patients or treat patients fairly

Illustration: That's really the focus of what we're doing here: patient care.... it comes down to whats happened with this particular patient". Kaldjian et al., (2006a) pg 945 [U]

\section{Category 2: Good rapport and established good relationship with the patient}

This category has three (3) findings and represents a positive rapport with the patient and having established a good relationship with the patient facilitates the disclosure of medical errors.

Finding 1: Communication success factor/Clinicians have a pre-established rapport with the patient (and family)

Illustration: "...we have already built a rapport with the [patient] so having an established rapport ...really added to the comfort and the trust, yeah." Iedema et al., (2007) pg 79 [U]

Finding 2: Disclosing Errors/Disclosing errors as a Decision 
Illustration: "This was the third day I had him. So we had already established a rapport. Probably, if it had been the first day I had him, he might not have been so...pleasant or understanding...But he really, I thought he did great with it, and I think it was because... he knew that I had been trying to take really good care of him the other two days. It was just the third day I had tried to hurry." Greene (2009) pg 72 [U]

Illustration: "she was a patient that I was particularly drawn to." Greene (2009) pg $73[\mathrm{U}]$

Illustration: "If you can get a rapport going with the family and patient, then it's a lot easier to tell them about things that happen." Greene (2009) pg 73 [U]

Illustration: "If they're friendly with me, and we have a good working relationship, and you talk back and to, it's easier. But if it's someone that's very quiet, maybe angry to start with, or ill, mad about being there, it would be more difficult.' Greene (2009) pg $74[\mathrm{U}]$

Illustration: "Its very hard to talk these patients, and hard to talk to them about error." Greene (2009) pg 74 [U]

Illustration: 'I would probably be more apt not to tell somebody that was a belligerent patient, that I had made a mistake, or that I had an oversight, or not given a medication on time...than a patient who is thankful that you're taking care of them, or who appreciates whatever you did for them, but those who just are constantly whining, and complaining, and just fussing about everything you do, I'd probably come down the side of telling that patient nothing, you know, versus the patient who is thankful for what you do for them.' Greene (2009) pg 74 [U]

Finding 3: Patient Factors/Rapport with provider

Illustration: "If doctors have a good relationship with their patients... patients really would relate well with their doctors. And medical errors, we could dialogue with a pretty decent way." Fein et al., (2005) pg 490 [U]

Illustration: "If you develop a good relationship over time with the patient and his family, if you are sincere and honest about if from the start, if you appear to be a caring human being they will excuse almost anything. If you are abrupt and short and 
you are dishonest, if you try to hide or you seem duplicitous and you seem uncaring and detached, then you are going to be in real trouble." Fein et al., (2005) pg 490 [U]

\section{Category 3: Desire to strengthen inter-professional relationships, build trust and support colleagues}

This category has five (5) findings and represents the desire of health professionals to develop positive inter-professional relationships based on mutual trust and supportive behaviour following a medical error.

Finding 1: Communication success factors/Staff maintain good interdisciplinary communication an relationships

Illustration: "Yes, we've got very good interdisciplinary relationships here. Whether it's an executive level or as you move down the line through the organisation, so I think we've got a very supportive culture of one another. In other words, it means you've got the opportunity to talk about Open Disclosure cases and be able to be fairly frank about it without feeling that you're going to be put down or criticized." Iedema et al., (2007) pg 79 [U]

Finding 2: Conducting Open Disclosure meeting: Managing staff who were most closely involved in the adverse event

Illustration: "If ... it's sort of like ... an obvious sharp end incident ... where the clinician has directly caused the harm, definitely they need so much more support, than when it's something that's indirect. ... So again, it all depends, some clinicians will need more support than ... others. I think it comes down to the actual nature of the incident, the disclosure." Iedema et al., (2007) pg 58 [U]

Finding 3: Following up: Providing adequate support for clinical staff (colleagues)

Illustration: 'I think it's extremely beneficial because you can talk about what went well, what went wrong, and you can talk about what you would do differently next time." Iedema et al., (2007) pg 69 [U]

Illustration: "[we have] an in-Unit sit down and talk about things particularly if people were upset ... and there are formal support mechanisms that you can access through the hospital for counselling and that sort of stuff if you need it." Iedema et al., (2007) pg 70 [U] 
Illustration: "I also keep my people after the person's left the room so that they can debrief, and they've got an opportunity to say, well, I really would have liked to have said that, or I was really angry when that person said, so that they actually get it off their chest in that environment rather than go away still feeling as if they... 'cause it actually can become quite a uh, combative environment." Iedema et al., (2007) pg 70 [U]

Finding 4: Organisation success factors/The organisation has efficient staff support measure in place

Illustration: “...we've got staff counsellors...I've got a clinical superintendent who I mobilise immediately for the medical officers who reports back to me and lets me know how the staff member is travelling. We give them time off if they need time off. The clinical director gets involved. So we've got resources and they're told all about the staff counselling." Iedema et al., (2007) pg 77 [U]

Finding 5: Responsibility to Profession/Desire to strengthen inter-personal relationships and build inter-professional trust

Illustration: Providing support to colleagues who are involved with errors. Kaldjian et al., (2006a) pg 945 [C]

\section{Category 4: Desire to be a role model and provide learning opportunities}

This category has three (3) findings and represents the importance of senior staff supporting open disclosure, promoting transparency, learning from errors and being a role model for colleagues.

Finding 1: Benefits Open disclosure/Open Disclosure may improve organisational culture

Illustration: ".....So they not only benefited from it, they learned from it and they're now teaching others." Iedema et al., (2007) pg 117 [U]

Illustration: "Well, I think inherently, the whole concept of a more transparent organisation, I think it makes for a healthier organisation.” Iedema et al., (2007) pg 117 [U] 
Illustration: "I initially had a few issues with my staff simply looking at me as a manager and saying whose side are you taking, sort of thing. ... I think, but all this is gone now." Iedema et al., (2007) pg 117 [U]

Finding 2: Responsibility to Professional/Desire to serve as a role model in disclosing errors or breaking bad news

Illustration: "When people have come out and told the patient, have taken responsibility-its usually based on just a need to do the right thing and the need to be a good role model for those who are training under you." Kaldjian et al., (2006a) pg $945[\mathrm{U}]$

Finding 3: Responsibility to Profession/Desire to share lessons learned from errors Illustration: "If I as a faculty member can't express my own fallibility... how can the learner learn"? Kaldjian et al., (2006a) pg 945 [U]

\section{Category 5: Open disclosure meetings are successful when health professionals} demonstrate effective communication and listening skills

This category has nine (9) findings and represents the perceived importance of effective communication and listening skills. Health professionals highlighted these skills as providing patients with the opportunities to express themselves and be reassured that their feelings and emotions are being heard and considered.

Finding 1: Conducting Open Disclosure Meetings -Active (reflective) listening Illustration: "The Standard thing is really just to listen to them [patients/family members], and allow them to develop their arguments and then to repeat it. I think that's probably the critical issue. If they have problems, then you go through them all and you repeat them to them so you're telling me these are the issues [to] get rid of the poison. ... I've had some very, very difficult patients, and you find at the end of it they will say, "thank you." Iedema et al., (2007) pg 52 [U]

Finding 2: Conducting Open Disclosure Meetings- ensuring that the Open Disclosure team maintains excellent communication standards

Illustration: "It is all about having the right people and I think that there are some people whose manner and interpersonal skills perhaps would be counterproductive in that sort of process. These people might be involved in the event. ... You can imagine 
someone [saying] 'I go off and do Open Disclosure with this patient'. And you think 'Oh, my god. Please don't' [or] 'Can I come with you'?’ Iedema et al., (2007) pg 53 [U]

Finding 3: Conducting Open Disclosure Meetings: Dealing with complex patientfamily dynamics

Illustration: [with] "The patient...you get everything right. But there's a relative ... out there who says ... 'that's what I would expect'." Iedema et al., (2007) pg 55 [U]

Finding 4: Conducting Open Disclosure Meetings: Preventing Open Disclosure from going wrong

Illustration: "[I was unable to] see the bloody question coming ... Too slow, I'm afraid. I put my hand up. Saw it, blindsided me, killed me. The exchange happened that quick that I was not on to it and, look, I don't know if I should persecute myself because it was foreseen in that pre- planning meeting and agreed, and when it still happened I was a bit annoyed." Iedema et al., (2007) pg 60 [U]

Finding 5: Perceived challenges/Staff do not sufficiently appreciate the emotional labour that is needed for Open Disclosure

Illustration: "Everything he [clinician] was saying was logical, it was rational, it had process behind it, but it wasn't empathic and he never kind of at all acknowledged the fact that she [patient] was hurting. That's all that she wanted to hear. That's all she wanted to hear." Iedema et al., (2007) pg 84 [U]

Finding 6: Perceived Challenges/Open Disclosure can exacerbate the clinicianpatient (family) relationship

Illustration: staff saying wrong or contradictory things' Iedema et al., (2007) pg 85 [U]

Illustration: 'staff unable to 'hear' patients' and family members' needs and feelings' Iedema et al., (2007) pg 85 [U]

Illustration: 'staff remaining unwilling to acknowledge the rights and emotions of the patient.' Iedema et al., (2007) pg 85 [U]

Illustration: 'staff proving unable to manage patients' (family members') distress and anger'. Iedema et al., (2007) pg 85 [U]

Finding 7: Pre planning the Open Disclosure session/Assembling the team 
Illustration: "you [might] get someone who's inexperienced running a [Open Disclosure] session, and that person might slip up and therefore be exposed." Iedema et al., (2007) pg 37 [U]

Illustration: “... if you are in a situation where you've got to do an open disclosure and you've got a choice between two clinicians to do it, then you're going to go for the person that has a better way of doing it." Iedema et al., (2007) pg 37 [U]

Illustration: "We make an assessment: we bring the doctor in and say 'so tell us all about it'. And if they're saying 'That bastard of patient did this and that and the other thing', they're not getting back near them [the patient]." Iedema et al., (2007) pg 37 [U]

Finding 8: Success Factors/Staff have good commutation abilities

Illustration: “ ... being mindful of the language ... if they looked puzzled then you address, 'do you not understand?' We don't say that but trying to ensure that they do understand what you're saying. [It's about] clarifying, getting them to rephrase and paraphrase and all that sort of stuff.” Iedema et al., (2007) pg 77 [U]

Finding 9: Success Factors/Staff are proficient in Active Listening or Reflective Listening

Illustration: "it's a fact that you don't just go and tell the patient, you have to sit there and just listen to the patient, and let them vent, let them tell you what they're thinking. And that's, that's the hard bit in Open Disclosure, is to be sat listening." Iedema et al., (2007) pg 73 [U]

Illustration: “... the family are livid, they're really, really angry. ... so I said ok, well we need to ... meet with them and talk through this. ... it took quite a while for them to just get rid of their anger. ... I didn't try and interrupt them or stop them from talking. I didn't try and deflect the blame because they were wanting to blame somebody, they were wanting to know the name of the nurse that was suppose to be looking after [the patient] ... for 25 minutes, they ranted and shouted and were very scathing of, of the service. I guess at the end of that ... they were just getting tired from being so angry, I ... really just apologised and said, you're right!' Iedema et al., (2007) pg $74[\mathrm{U}]$ 
Category 6: Desire to educate the patient about the complexities and associated risk of health care and help promote patient trust

This category has five (5) findings and represents the health professionals expressed desire to help patients and the community to gain a deeper understanding that health care is becoming increasingly complex and that there are risks associated with the provision of care.

Finding 1: Benefits of Open Disclosure/may assist with healing

Illustration: "Well, health care is a trust game. ... If you undermine the trust, you undermine the therapeutic relationship, which is important for the patients as well as it is for the comfort of the staff. If they don't feel like they can trust it's much harder for them to get better." Iedema et al., (2007) pg 116 [U]

Finding 2: Benefits of Open Disclosure/may lead to much better relations with patients and family members

Illustration: “ "... when you actually saw that it worked on occasion, you actually got letters of thank you, you know from people who, you'd told horrible stories to about things that you had done to them or their families, um, you know, so, um, so that enabled us to continue to do it." Iedema et al., (2007) pg 118 [U]

Finding 3: Responsibility to Community/desire to help patients understand the complex causes of errors

Illustration: 'To educate the community about medicine's complexities and imperfections.' Kaldjian et al., (2006a) pg 945 [C]

Finding 4: Responsibility to Community/desire to sustain patient's trust in the medical profession.

Illustration: 'To enhance society's trust in physicians and the medical profession'. Kaldjian et al., (2006a) pg 945 [C]

Finding 5: Success factors/Extended formal consent and public education

Illustration: "Tell patients about the bad things that can happen up front, so when they do happen it's, 'Oh.' 'Sometimes people get the wrong leg cut off'. 'I got the wrong leg cut off'. It's not a surprise. It happens. I mean we have these ridiculously long consent forms and all that sort of stuff but ...we only have consent forms for procedures. When you come in with pneumonia, you never sign a consent form but 
you might get an infected cannula and end up having your finger cut off because of some terrible infection and you get a clot in your legs. Maybe there needs to be some sort of overriding statement [about these additional treatment risks]. You might come into hospital, you might have a drug error, you might have this happen ... so when they do happen, 'Oh, yeah, well this is part of the risk of being sick and being treated'. Iedema et al., (2007) pg 81

\section{Category 7: Perceived benefits of open disclosure}

This category has three (3) findings that represent the sense of relief felt by health professionals for having a framework to disclosure medical errors to patients. The formal meeting provides support, has a positive impact, promotes patients trust and decreases the number of patient complaints.

Finding 1: Benefits of Open disclosure/may lower compliant levels

Illustration: "Yes. I think it's been successful ... Certainly from one of the health service's perspective ... that has had huge success in terms of one of their areas and overall in the hospital [name] was saying that their complaints have gone down $42 \%$ which is amazing but whether that just relates to this or not who's to know without doing more evaluation.” Iedema et al., (2007) pg 116 [U]

Finding 2: Benefits of Open disclosure/Provide closure

Illustration: "Well, clearly, the big thing is closure for, an attempt at closure. You're probably never going to get a hundred percent closure but you at least get people to be able to get back to moving on with their lives, not stuck in a time warp about an incident and becoming bitter and twisted which can have an effect for the rest of their lives.” Iedema et al., (2007) pg 116 [U]

Finding 3: The perceived Benefits of Open Disclosure/approval of Open Disclosure as policy principle

Illustration: "Everybody that's been involved with it have felt quite relieved." Iedema et al., (2007) pg 18 [U]

Illustration: "I think in some ways they [staff] are relieved because ... there is a plan: this is what we are going to do with this family." Iedema et al., (2007) pg 18 [U] 
Illustration: "We had a massive case of an absolutely horrendous situation involved and we went through an Open Disclosure process and that was the most amazingly kind of positive experience.” Iedema et al., (2007) pg 19 [U]

\section{Category 8: Desire to be accountable for ones actions}

This category has three (3) findings and represents having the courage and willingness to accept accountability for ones actions and inform patients about errors. Finding 1: Organisational success factors/Senior staff participates in Open disclosure Illustration: "Well, I think senior clinician involvement is absolutely vital to make it work, and that ethos that it's okay to talk to the patients." Iedema et al., (2007) pg 77 [U]

Finding 2: Responsibility to self/desire to be accountable for ones actions

Illustration: "being accountable for our errors and not being a weasel or arrogant or denying that we ever make errors." Kaldjian et al., (2006a) pg 945 [U]

Finding 3: Responsibility to self/Desire to be courageous

Illustration: "If you don't have the guts to say, "I screwed up" to a patient, you're in the wrong business." Kaldjian et al., (2006a) pg 945 [U]

\section{Category 9: A desire to seek forgiveness and alleviate guilt}

This category has four (4) findings and encompasses the emotional need to seek forgiveness, alleviate feelings of guilt and an opportunity to explain to the patient the circumstances surrounding the medical error.

Finding 1: Physicians' emotions following an error/relief

Illustration: '[We are] trying to relieve the soul of some burden when we confess our sins or our errors... and dumping that onto the patient is not necessarily nice'. Gallagher et al., (2003) pg 1005 [U]

Finding 2: Physicians' Emotions following and Error/challenge was forgiving themselves

Illustration: 'It helps if the patient says, "Look, I understand that this is not normal, but I am willing to go along with whatever you say.... and to give you that extra support and second chance." Forgiveness is something that I think is tougher for the 
physicians to give themselves that to get from the patient.' Gallagher et al., (2003) pg $1005[\mathrm{U}]$

Finding 3: Responsibility to patient/Desire to explain to patient the circumstances surrounding an error

Illustration: Emotional responses to errors such as the desire to explain circumstances surrounding an error. Kaldjian et al., (2006a) pg 945 [U]

Finding 4: Responsibility to Self/desire to alleviate guilt or pursue forgiveness

Illustration: "in order to receive forgiveness you have to admit to your wrong." Kaldjian et al., (2006a) pg 945 [U]

Illustration: "make amends" Kaldjian et al., (2006a) pg 945 [U]

\section{Category 10: Desire to be honest or do the right thing}

This category has three (3) findings, and represents the desire of health care professionals to follow their conscience, to do the right thing and be honest with the patient by informing them about a medical error that has occurred.

Finding 1: Communication success factors: Staff display and enact sincerity

Illustration: “...it's still a very emotional, draining, stressful situation that ... you actually go through, and, you really have got no idea when you walk into that meeting. All it's left up to ... is your good communication skills ... but ... most people are going through life-altering change, and they're in crisis, and you don't really know how the people are going to react ... so that's what sharing the pain is about." Iedema et al., (2007) pg 75 [U]

Illustration: "There has to be a sense of trust because often what you're dealing with is recovering from a position of trust which has been lost. So any commitments that you make you have to keep. People have very well-tuned bullshit sensors and you've got to be very careful about being absolutely direct and honest. Which is why we're careful about who we let do it because [if] people sense that you're spinning a story rather than information their bullshit sensors go off and you've lost it." Iedema et al., (2007) pg 75 [U]

Finding 2: Disclosing Error/Disclosing Error through Constant Communication 
Illustration: "You can't just make an error and let it go, because they [patients] need to know, I mean it's their life... if it was me I would want to know. People make mistakes." Greene (2009) pg 69 [U]

Illustration: 'Be honest regardless of what the consequences are...You know, even if something were to happen, and I was in front of a judge, and I told him "yeah I was honest," I believe he would look at that a lot better than me keeping that from him and the family or patient. Always be honest. That's what I was taught, and I still believe that.' Greene (2009) pg 70 [U]

Finding 3: Responsibility to Self/Desire to follow ones conscience or 'do the right thing'

Illustration: 'Personal and professional values that derive from the physicians character.' Kaldjian et al., (2006a) pg 945 [C]

\section{Category 11: A culture of honesty}

This category has three (3) findings and represents the perception amongst health care professionals that a culture of transparency and honesty within an organisation facilitates the open disclosure of medical errors to patients by health professionals.

Finding 1: Benefits of Open Disclosure/Open Disclosure may improve organisational culture

Illustration: ".....So they not only benefited from it, they learned from it and they're now teaching others.” Iedema et al., (2007) pg 117 [U]

Illustration: "Well, I think inherently, the whole concept of a more transparent organisation, I think it makes for a healthier organisation.” Iedema et al., (2007) pg 117 [U]

Finding 2: Open Disclosure viewed in positive terms

Illustration: "Well, all my experience with [Open Disclosure] is positive. It is contributing to the culture thing, it is about getting it off people's chest thing, there is no dealing of hidden agendas, there is no feelings of [distrust], there is true transparency.” Iedema et al., (2007) pg 115 [U]

Finding 3: Perceived Expectations for Disclosure/Cultures of Openness and Honesty 
Illustration: "At first we notify the physician, and with the policy, on the report form it asks "was the patient informed?" and "was the physician notified?" and you have to check "yes." You have to put the time and the response to it [disclosure]." Greene, (2009) pg 78 [U]

Illustration: "I think they [managers] would be fine with it... I think they would support us....if you made a medication error, and we [nurse] went to the patient and told them. I think they would not have a problem with that" Greene (2009).pg 80 [U] Illustration: "its going to be better if it came from you, and you let them know, 'this is what I've done to fix the problem."' Greene, (2009) pg 81 [U]

\section{Category 12: Integrated processes, and service to support staff}

This category has six (6) findings and represents system wide processes to manage medical errors and services to support staff, facilitates open disclosure.

Finding 1: Acknowledging and communicating errors

Illustration: "then, we had to report the occurrence, which is through a form we have here at the hospital for when an error occurs...he (supervisor) forwarded it to the Ethics Committee and the families were informed, everything was all right, everything was according to the legal process as it is supposed to be." De Cassia Pires Coli, et al., (2010) pg 328 [U]

Finding 2: Conducting Open disclosure/distinguishing between conventional ways to deal with unexpected outcomes and the practices required by open disclosure

Illustration: "I suppose that previously we wouldn't necessarily have gone and had anyone else involved such as the [Support Personnel]. It would have been more informal within the Unit situation where we sit down and discuss with the patient and [address] their concerns, so I suppose it's a lot more formalized now than it used to be." Iedema et al., (2007) pg 57 [U]

Finding 3: Following up: creating and maintaining organisational memory

Illustration: "I think they [Open Disclosure and Root Cause Analysis] are [mutually exclusive] because in a Root Cause Analysis there is a protection, 64D ... against any information that is discovered during the investigation ... that means that nothing can be disclosed. Whereas I see Open Disclosure as being more a process where the 
information is actually not hidden, it gets disclosed to the patient and it is disclosed to everybody really.” Iedema et al., (2007) pg 70 [U]

Finding 4: Institutional Culture/Supportive Infrastructure

Illustration: "There needs to be a culture where individuals do not feel penalized for reporting errors. You should feel comfortable reporting to the chief of service or the head of nursing." Fein et al., (2005) pg 492 [U]

Finding 5: Organisational success factors/Organisational-structural prerequisites have been put in place.

Illustration: “.....the time you prepare for Open Disclosure is years back. You try and ... work with policies and procedures and frameworks that are already in place and that people are familiar with.” Iedema et al., (2007) pg 79 [U]

Finding 6: Staff are supported in confronting legal, insurance and professional (reputation) uncertainties

Illustration: "Yes... we certainly do admit liability when we've done the wrong thing. We do it in a controlled way, though. We will check with our insurer first, because we want to be sure that we're indemnified. We will all have a good think about whether we're going to create a fresh wave of innocent victims, which is always possible if you use the wrong words and do it the wrong way." Iedema et al., (2007) pg 50 [U]

\section{Category 13: Pre planning facilitates the success of open disclosure}

This category has four (4) findings and represents the importance of pre planning, the gathering of facts about the incident and appraising all the information prior to conducting an open disclosure meeting. Similarly, anticipating the responses of people in the meeting, and discussing strategies to effectively manage the intensity of the situation.

Finding 1: Pre Planning/Assessing the dynamics of the patient/family

Illustration: We really just try and predict what sort of things might be their concern and see if we can understand the facts around what their concerns might be so that we can actually explain it well enough back again.” Iedema et al., (2007) pg 38 [U] 
Finding 2: Pre Planning/Planning language to use and practicing how to apologize given the specifics of the adverse event (without jeopardizing the authenticity of the meeting)

Illustration: We sit down and go through who's going to do what and organize who's going to say what." Iedema et al., (2007) pg 39 [U]

Finding 3: Pre planning the Open Disclosure session/Assembling the team

Illustration:“... what we try and do is, consistent with the policy, we have a team of three for the major disclosure processes. So we ideally have the senior clinician involved in the case, a patient representative/client liaison officer, and a representative of the administration for the hospital or wherever the incident occurs." Iedema et al., (2007) pg 37 [U]

Illustration: "If there are apologies to make and I thought they [junior staff] were competent [to do that], and there are some that are competent, I would involve them in that process. But in general terms most of my difficult patients ... come back to a special clinic where I have control of that situation.” Iedema et al., (2007) pg 38 [U]

Finding 4: Pre planning the Open disclosure session/Understanding the adverse event Illustration: Usually we'll talk to the person [the clinician most closely involved in the adverse event] and make sure we've both got a common understanding of what the event entailed and make sure we've both looked at the history and the medical record and make sure that the Nurse Unit Manager has got a handle on all the nursing issues and I'm okay with all the medical issues [because] we've talked to the relevant people.” Iedema et al., (2007) pg 36 [U]

\section{Category 14: Services that support patients who have been involved in a medical} error

This category has two (2) findings and represents the availability of adequate resources and services within an organisation to support the needs of patients who have been involved in a medical error.

Finding 1: Communication success factors/Bereavement counseling benefits patients and family members 
Illustration: "And that's why I think very, at the beginning when we identify that there has been a serious adverse event that we put in place bereavement support, the families can start to separate out the clinical bereavement response from the feedback process.” Iedema et al., (2007) pg 80 [U]

Finding 2: Communication success factors/Staff enact restorative justice

Illustration: 'Now, a lot of people actually can find the question slightly offensive to even talk about money in the middle of that meeting, so it's got to be brought up sensitively, that says, Look, we have the ability to assist you with some out-of-pocket expenses'. Iedema et al., (2007) pg 76 [U] 


\section{Chapter 4: Discussion}

\subsection{Overview of findings}

The objective of the review was to investigate the perceptions and experiences of open disclosure of medical errors to patients by health professionals. The review findings were synthesised into two (2) comprehensive meta-syntheses aiming to inform the two (2) specific questions of the review: What are the barriers to open disclosure of medical errors to patients by health professionals? What are the facilitators to open disclosure of medical errors to patients by health professionals?

The review process identified a greater number of studies and findings informing the barriers to open disclosure as opposed to the facilitators of the practice. The synthesized findings of this review, derived from pertinent qualitative research, reveals the difficulties health professionals experience informing patients and their family of errors in medical care. Delivering bad news of the potential consequences that may be associated with medical errors is confronting, and most health professionals preferred to avoid this stressful situation.

Identified barriers to open disclosure included professional and personal emotions and feelings, circumstances specific to the error, uncertainties of a clear definition of medical error, organisation culture and system factors. In addition, intolerance, blame, perfectionism and a lack of education in the management and disclosure of medical errors amongst health professionals also acted as barriers to the disclosure of medical errors to patients by health professionals. The diversity of the patient population and the health care workforce raises cultural issues that challenge perceptions and expectations of communicating errors in health care.

The facilitators of open disclosure include a sense of responsibility, having a good rapport with the patient, and established relationship with the patient. The level of cooperation amongst health professionals and their desire to promote and develop 
interdisciplinary relationships also impacted on whether patients were informed about errors. Other facilitators included wanting to learn from medical errors, being a role model for others, improving communication and educating the public about the increasingly complexity of health care and the associated risks. In addition, the perceived benefits of disclosing medical errors to patients encouraged health professionals to be more willing to advocate for the disclosure of errors to their patients.

\subsubsection{Barriers to open disclosure}

The first question posed by this review is addressed by meta-synthesis one (1). The individual barriers to open disclosure that have been identified in this review and form meta-synthesis one (1) were numerous and complex in nature.

The findings of this systematic review identified a range of personal fears that prevented health professionals from disclosing errors to their patients. Health professionals failed to disclose medical errors to patients to protect themselves from the discomfort of engaging in conversations with their patients about errors. They were motivated not to disclose errors to avoid feelings of distress when confronted with the angry reactions of patients or those of their family.

The decision whether to disclose errors to patients was further complicated by a desire to protect oneself from the negative consequences of disclosure and the moral and professional responsibility to inform patients about errors. When confronted with this dilemma, health professionals often experience a range of intense emotions, such as fear, anxiety, shame, guilt, self-doubt and humiliation. Physicians particularly feared admitting to not only the patient, but to their colleagues and to themselves that they had made a mistake. Fear of a loss of confidence, self-esteem and doubts of their suitability to practice as a health professional increased their reluctance to disclose errors to their patients. 
Studies by Christensen et al., 1992; Hingorani et al., 1999; Gallagher et al., 2006a; Ghalandarpoorattar et al., 2012; and Linthorst et al., 2012 surveyed physicians and reported similar reasons for non disclosure of medical errors to patients. Physicians particularly feared being judged as incompetent by their colleagues. They reported open disclosure as being too difficult, and not disclosing errors protected them from facing the patients' anger that they anticipated would be directed at them. Health professionals feared the loss of the patients' trust, with tension eroding their relationship with the patient. The literature also suggests that a lack of transparency about errors by health professional can lead to the patient losing faith in their clinical safety and the professionalism of the staff caring for them (Vincent, 1998). Although decisions to disclose errors are often complex for health professionals, failures in patient expectation are often reflected in lower patient satisfaction and perception in the quality of the health care system (Gallagher et al., 2003; Witman et al., 1996).

Similarly, health professionals experience feelings of self-doubt and disappointment when reflecting on their willingness to reveal errors to patients (Waterman et al., 2007). The findings of this review revealed fear of litigation acted as a strong barrier to disclosure, especially amongst physicians who held concerns of being vulnerable to legal action being taken against them. These findings are well supported in the literature; Studdert et al., 2010 reported $88 \%$ of respondents considered medico-legal risks as a major or moderate barrier to open disclosure, and Mariner (2001), suggests health professional are reluctant to discuss errors with their patients because they believed they have no appropriate assurance of legal protection in the event that the patient decided to take legal action against them.

A lack of confidence in knowing how to initiate conversation about errors with their patients often prevented health professionals from revealing errors. They described situations in their clinical practice where they felt overwhelmed and unprepared to meet the expectations of patients who want to be informed about errors in their health care. A lack of adequate training and appropriate education in open disclosure also left them fearful of looking foolish in front of their junior colleagues, and long term 
damage to their reputation. This review revealed that a recurrent perception amongst health professionals was that by informing patients of errors, would subject them to judgments of incompetence, not only from the patient, but also from colleagues. They were fearful of being labeled as someone prone to making mistakes, and risk further career opportunities or perhaps even ultimately their current job.

Rationalisation was employed across the health professions to justify the reasons why they did not disclose errors. Health professionals believed they had a responsibility to help minimise the patients' level of anxiety and stress during any health care encounter. Therefore, they justified not disclosing medical errors to their patients out of a desire to protect the patient from what they perceived as unnecessary anxiety and stress from knowing that there had been an error in their care. However, failure to disclose details to patients may be considered an attempt to protect their own emotional survival as much as to protect their patient. The literature suggests that doctors frequently censor information they gave to their patients on the grounds that what someone does not know cannot hurt them (Fallowfield and Jenkins, 2004). Such traditional paternalistic attitudes are still encountered despite moves towards increased patient autonomy and empowerment, and prevent patients from being informed about medical errors (Ghalandarpoorattar et al., 2012).

The findings of this systematic review showed that despite health professionals having conversations with their patients following an event in care, the level of detail was sometimes limited and stopped short of disclosing an error. The non-disclosure of errors through the use of selective language and behaviour acted as a barrier to open disclosure. Health professionals described 'choosing their words carefully', or putting the most 'positive spin' on the situation in cases when they were communicating to patients about outcomes of care. Additional details may be disclosed if the patient or their family member asked directly. However, some patients may readily accept the selected information provided to them, while others may want to know more but lack the confidence to ask, leaving them to reflect and try to 'connect the dots' to reach their own conclusion of what occurred during their care. 
Other scenarios of non disclosure involved conversations which left patients with the impression a negative outcome in their care was the outcome of a natural progression of their disease or a complication of their treatment. This finding is supported in the study by Gallagher et al., 2006b, which reported that $56 \%$ of physicians did not tell the patient that there had been a medical error in their care, and $13 \%$ of physicians reported that they would not volunteer any details about an error to the patient unless the patient asked them directly. Historically, patients have not been encouraged to speak up and ask questions about their care, and may be reluctant to voice their suspicions of any error to health professionals (Mazor et al., 2012). Similarly, the impact of mistakes and how health professionals cope has been examined in the literature. Three general behavioural techniques of denial, discounting, and distancing themselves from the mistake are employed as effective coping mechanisms to manage their emotions after making a mistake during their practice (Wu et al., 1993; Smith and Forster, 2000; Richman et al., 2009). Therefore, the lack of acknowledgement of mistakes results in errors remaining hidden from patients.

The circumstances surrounding an error impacted health professionals' decision to discuss errors with patients. The level of harm the patient experienced from the error and the consequences the error had on the patient affected their willingness to tell the patient about an error. Health professionals failed to disclose errors if they believed the patient and their family did not suspect an error. A study by Weissman et al., 2005 reported $84 \%$ of participants would disclose errors causing serious harm, compared to $38 \%$ with no harm to the patient. Loren et al., 2008 surveyed paediatricians asking them in what circumstances would they disclosure a medical error, this professional group reported they would only disclosed medical errors if a parent of the child was aware that an error had occurred.

Medical errors causing no harm, immediate or long term consequences for the patient are often referred to as a 'near miss'. There is much debate in the literature of the value of disclosing these types of errors to the patient and whether it is necessary for the health professional to do so (Piper and Iedema, 2011). Patient safety experts often 
consider near misses to be red flags to underlying issues posing a risk to patient safety, requiring attention and should not be ignored (Kohn, et al., 2000). However, some would speculate it is beyond the call of duty of health professionals to communicate to patients about problems in care (Piper and Iedema, 2011).

Although an error may be considered a 'near miss' by the health professional and not worth disclosing, the event may have produced suffering or harm in the eyes of the patient or family (Iedema et al., 2011a.) Health professionals should not assume patients do not want to be informed about errors just because they did not sustain direct harm. Despite this, Gallagher et al., 2006b and Dintzis et al., 2011 demonstrated $17.8 \%$ - 30\% of participant surveyed reported they would not disclose an error if they thought the patient would not want to know about it.

The findings of this systematic review found an apparent lack of a consistent definition of what constitutes an error in the process of care, prevented disclosure. Similarly, uncertainties surrounding what errors should be disclosed to the patient, acted as barriers to disclosure. Different views are often held amongst physicians even within the same specialty of medicine regarding what is considered to be an error. Some physicians may explain the patient's condition as an unpreventable complication of treatment or the patient's underlying disease rather than a direct outcome of an error in care.

Uncertainties surrounding when a medical error was significant enough to be disclosed to the patient prevented open disclosure. Banja, 2008 highlights, that an inadequate error definition can create organisational confusion and raise conceptual questions amongst health professionals of whether an error has actually occurred or not and uncertainty of what errors to disclosure to patients. This is further complicated as patients and clinicians hold very different views on what constitutes a medical error and expectations of disclosure (Iedema et al., 2008; Dauer, 2011). 
The culture of an organisation played a significant role in the incidence of open disclosure, cultures of secrecy and silence, inevitably resulting in medical errors being hidden and concealed from patients. This review revealed that the absence of policies specifically supporting the disclosure of errors created a lack of confidence in the integrity of the organisation to support staff prepared to disclosure errors. The perceptions of a lack of support for staff left health professionals feeling powerless to inform their patients about errors. Furthermore, expectations of perfectionism, intolerance for errors and a culture quick to place blame on the individual involved in an error, all acted as barriers to open disclosure of medical errors to patients (Allman, 1998). In addition, the medical profession is often expected, coupled with high selfexpectation, to consistently operate at peak performance levels free of mistakes or errors in their practice. These expectations and cultural demands within the medical profession appear to exert the strongest influence over physician decisions to disclose medical errors or not, therefore imposing boundaries and constraints that are inherent of the profession (Allman, 1998; Smith and Forster, 2000).

Health professionals experienced the challenge of how to make the process of open disclosure culturally sensitive and relevant in light of global migration of patient and clinical staff. Furthermore, the diverse cultural and linguistic background of patients means some sections of the patient population may have a lower rate of health literacy, which may impact how they experience medical care, errors and disclosure.

Moreover, system factors acted as barriers to the practice of open disclosure, the view amongst health professionals was that open disclosure takes too much time. High workloads, professional shortages and measures aimed to enhance efficiency with fewer resources limited the time health professionals are able to spend with their patients. Although this finding is supported in the literature, it is acknowledged that by failing to prioritise the allocation of specific time to disclose errors to patients, the health professional-patient relationship is placed under pressure and can be the catalyst for its breakdown (Smith \& Forester 2000; Cravens \& Earp 2009). 


\subsubsection{Facilitators of open disclosure}

The second question posed by this review exploring what health care professionals experienced and/or perceived as the facilitators to open disclosure is addressed by meta-synthesis two (2). The findings of this systematic review indicated health professionals perceived having a good rapport and an established relationship with their patient facilitated open disclosure of medical errors. Being open and telling the truth was considered an integral part of this relationship and despite concerns of negative consequences health professionals informing patients about errors was part of their clinical practice. Dintzis et al., 2011 reported that disclosure of pathology errors was complicated and prevented by the absence of an established relationship between the pathologist and the patient. Other factors facilitating disclosure included health professionals wanting to develop and promote a strong interdisciplinary relationship based on principles of trust and supporting colleagues involved in errors.

Historically, the disclosure of medical errors has been considered an interaction that took place between the physician and the patient (Boyle et al., 2006; Luce, 2006). However, nurses play a pivotal role in promoting the effective communication of information to the patient and hold a shared accountability for errors that may occur during the delivery of health care (Shannon et al 2009). Shannon et al., 2009 suggests nurses routinely disclose nursing related errors to their patients independently of physicians, such as a late or missed medication, delays in treatment, failures in the coordination of care, or failure to communicate patient information during nurse to nurse handover. Although nurses experienced a level of independence in disclosing nursing related errors, it was still the expectation of nurses that physicians would lead the disclosure of errors involving serious harm or errors from the actions of other members of the health care team (Shannon et al., 2009).

However, nurses considered a collaborative approach to open disclosure of these types of errors as ideal, to ensure that accurate information was provided to the patient regarding the nursing role in the event (Shannon et al., 2009). Despite this, in a study by Wagner et al., 2012, it was reported $54 \%$ of nurses would not disclose full 
details to the patient and/or their family following a medical error in the patients care. Nurses' hesitation to disclose errors made by other health professionals such as physicians may reflect the perceptions of role expectations and professional boundaries with physician holding the authoritative power regarding the disclosure of some errors.

The literature suggests education and training are essential requirements to prepare health professionals to engage in conversations with their patients about medical errors (Kaldjian et al., 2006b). Effective listening and communication skills have been identified in this systematic review as being particularly beneficial in facilitating the process of open disclosure. The management of errors including the disclosure of medical errors to patients has been defined by Christmas and Ziegelstein, 2009 as the seventh core competency of medical training, who suggest that teaching medical students how to manage errors will improve the quality of care, patient safety and improve patient satisfaction with the health care system. Similarly, studies by Gunderson et al., 2009 and Bonnema et al., 2009 suggest that practitioners with disclosure training feel more prepared, confident and comfortable with disclosing errors to their patients, supporting the findings from this systematic review. Furthermore, the active participation and support of senior health professionals in the open disclosure process provides positive role models for all health professionals and acts as a facilitator to disclosure of medical errors to patients.

The findings of this systematic review showed education of patients, their family members, and the wider community facilitated the open disclosure process. Providing education creates early consideration and awareness amongst patients of the risk of something going wrong, helping to eliminate unrealistic and flawed patient expectations. Conversations with patients about the risk of errors creates an awareness that may alleviate tensions between patients and clinical staff when it comes to having difficult conversations about medical errors (Kalra, 2004). 
Health professionals' perceived the benefits of open disclosure as being a reduction in the number of patient complaints, a higher level of patient satisfaction and providing patients with a sense of closure after being involved in a medical error. Lopez et al., 2009 reported the disclosure of medical errors to patients resulted in a $95 \%$ rating by patients of the services and the quality of care provided to them. Similarly, health professionals described positive experiences and feeling a sense of relief by having effective guidelines to assist them through the process of disclosing errors to their patients.

Other facilitators identified in this systematic review involve the motivation of health professionals to meet their own personal needs, such as a desire to be accountable for their own actions, and do the right thing. In the aftermath of being involved in a medical error, health professionals experienced feelings of guilt and the need to seek forgiveness. By telling the patient what happened and disclosing the error, health professionals describe experiencing a sense of release, to unburden themselves from feelings of guilt and shame. Similarly, admitting to involvement in an error without fear of retribution provided them with a greater sense of confidence in the organisation. In a study of physicians, Wu et al., 1991 similarly reported that doctors were often relieved after admitting to a serious error, and their feelings of guilt were diminished by disclosing the error to the patient or family and seeking their forgiveness. Furthermore, interdisciplinary relationships based on trust and honesty created supportive environments where health professionals felt comfortable in discussing errors and disclosing them to patients.

System related factors perceived by health professionals to facilitate open disclosure include the implementation of and compliance with effective frameworks to guide the disclosure of errors to patients. Structured pre planning meetings, and supportive services for patients and staff were considered essential components for successful open disclosure. Effective open disclosure frameworks supported health professionals, giving them confidence to effectively manage the potentially emotionally charged, and unpredictable situations when disclosing errors to patients 
and their family. Research suggests that adequate preparation for the disclosure provides time for the health professional to deal with their own emotions and they are more likely to consider the needs of the patients over their own needs, and provide a more successful and compassionate message about medical errors (Petronio et al., 2013).

\subsubsection{Global perspective}

Internationally, open disclosure is recognised as promoting patient safety, patient expectations and reducing litigation following medical error. There has been a steady move towards the development and implementation of standards, policies and frameworks to help achieve consistency in practice and guide organisations and frontline staff in the process. Various disciplines have discussed disclosure from their own professional perspective, with some generating practical, evidence-based guidelines (Hannawa et al., 2013). These strategies have a number of shared characteristics, all profile open disclosure as the right thing to do, are patient centered and support the disclosure of medical errors that cause harm to the patient.

\subsection{Limitation to the study}

The search strategy for this systematic review was limited to studies reported and published in the English language. It is recognised evidence published in languages other than English, and not included, may have added to the findings. Furthermore a lack of a consistent definition of medical error may have impacted on the search strategy.

The studies included in this review involved research including participants from the acute hospital setting, therefore, it is uncertain if all the findings, particularly the system factors that were identified as barriers or facilitators would translate into other health care settings such as primary health care settings. 


\subsection{Implications for practice}

The review included studies of a high methodological quality by the critical appraisal process, providing confidence in the quality of the evidence, and the findings from this systematic review. Therefore, the interpretation of the findings and the final meta-synthesis provides credible evidence useful to inform clinical practice and assist policy makers to develop strategies to remove barriers and promote the facilitators.

Evidence emerging from this review indicates health professionals experience a variety of factors acting as barriers and facilitators to open disclosure of medical errors to their patients. A significant gap between patient expectations and what is currently being experienced has been well documented. The international support and move towards open disclosure is providing awareness of the importance of open disclosure and driving momentum for the development of guidelines for organisations to effectively manage open disclosure of medical errors to patients. Guidelines aim to improve the current practice of open disclosure, to establish a consistent approach to facilitate the management of open disclosure in a timely, supportive and effective manner for patients, family members and all health professionals. Furthermore, guidelines provide a clear process describing the responsibilities of organisations and individuals in the management of open disclosure including the consideration of legal implications. Therefore, by developing strategies to remove some of the recognised barriers such as fear of litigation, uncertainties, not knowing how to disclose errors, a lack of support and organisational culture, the practice of open disclosure is supported.

In addition, efforts to remove the barriers and promote the facilitators should continue through effective education, role modeling and promoting cultures of honesty and transparency. Even if the implementation of system related factors was successful in diminishing some of the barriers to open disclosure, it will remain an intrinsic challenge at the level of the individual health professional to disclose errors to their patients. Health professionals need to address their own emotional responses and needs that act as barriers to open disclosure. For there to be significant sustained 
progress health professionals need to cultivate values that are capable of meeting the challenge to address the barriers to open disclosure. There also needs to be ongoing efforts to continue the education of health professionals in how they regard open disclosure of medical errors to their patients and how it is conducted. Preparation and planning prior to the disclosure of errors is highly recommended, it provides time for the circumstances of the error to be examined while providing support for those involved in the error. This time is considered an opportunity for those involved in an error to deal with their emotions before facing the patient. Similarly, a structured process to guide open disclosure meetings helps reduce the untapped reactionary emotions that may be stimulated once coming face to face with the patient and/or family and prepares staff for the potential wave of emotions a patient and their family may direct at them personally.

Facilitators of open disclosure such as cultures of honesty, interdisciplinary relationships based on trust also promote patient safety. Organisations should be working towards promoting these facilitators, with a cultural shift from a punitive culture to one where health professionals feel supported by the organisation and their colleagues following an error.

\subsection{Implications for Research}

Open disclosure is a recent development in health care and further research is required to review its implementation into clinical practice. The views and experiences of patients and their families with open disclosure will have an impact on the success or failure of the implementation of an open disclosure process in hospitals. Therefore, a systematic review on the patients' perspective of open disclosure to investigate if open disclosure is meeting the needs of patients and their family members would be ideal to further inform policy and decision makers to work towards ensuring the disclosure of medical errors is patient centred. 


\section{References}

ABHOLZ, H., FISSENI, G., PENTZEK, M. 2008. Responding to Serious Medical Error in General Practice-Consequences for the GPs involved: analysis of 75 cases from Germany. Family Practice An International Journal, 25, 9-13.

ALLMAN, J. 1998. Bearing the burden or baring the soul: physicians' self-disclosure and boundary management regarding medical mistakes. Health Communication, 10, 175-197.

AMERICIAN SOCIETY FOR HEALTHCARE RISK MANAGEMENT, 2003.

Disclosure of unanticipated events: creating an effective patient communciation policy (available http://www.ashram.org/ashram/education/development/monographs/monogra ph-disclosure2.pdf accessed 21 March 2012)

AUSTRALIAN COMMISSSION ON SAFETY AND QUALITY IN HEALTH CARE, 2008. Open Disclosure Standard: A National Standard for Open Communication in Public and Private Hospital, following an adverse event in health care. Healthcare (available http://www.safetyandquality.gov.au/wpcontent/uploads/2012/01/OD-Standard-2008.pdf accessed 20 March 2012)

AUSTRALIAN COMMISSION ON SAFETY AND QUALITY IN HEALTH CARE, 2013. Open disclosure principles, elements and process. (available http://www.safetyandquality.gov.au/wp-content/uploads/2013/05/Opendisclosure-principles-elements-and-process.doc accessed 15 July 2013)

BANJA, J. D. 2008. Problematic medical errors and their implications for disclosure. HEC Forum, 20, 201-213.

BONNEMA, R. A., GOSMAN, G. G. \& ARNOLD, R. M. 2009. Teaching error disclosure to residents: a curricular innovation and pilot study. Journal Graduate Medical Education, 1, 114-118.

BORBASI, S. A. 1996. Capturing the experience of the clinical nurse specialist through phenomenology in Qualitative Research Practice in Adult Education. (eds) Willis, P. and Neville, B. David Lovell Publishing, Ringwood, Victoria.

BOYLE, D., O'CONNELL, D., PLATT, F. W. \& ALBERT, R. K. 2006. Disclosing errors and adverse events in the intensive care unit. Critical Care Medicine, 34, 1532-1537.

BYRTH, J., AROMATARIS, E. \& MC ARTHUR, A. 2012. Health professionals' perceptions and experiences of open dislcosure: A systematic review of 
qualitative evidence. JBI Library of Systematic Reviews (Assessed 20 Aug 2013).

CHAMBERLAIN, C. J., KONIARIS, L. G., WU, A. W. \& PAWLIK, T. M. 2012. Disclosure of "nonharmful" medical errors and other events: duty to disclose. Arch Surgery, 147, 282-286.

CHRISTENSEN, J. F., LEVINSON, W. \& DUNN, P. M. 1992. The heart of darkness: the impact of perceived mistakes on physicians. Journal General Internal Medicine, 7, 424-431.

CHRISTMAS, C. \& ZIEGELSTEIN, R. C. 2009. The seventh competency. Teach Learn Medical, 21, 159-162.

CLINTON, H. R. \& OBAMA, B. 2006. Making patient safety the centerpiece of medical liability reform. New England Journal of Medicine, 354, 2205-2208.

CONWAY, J., FEDERICO, F., STEWART, K. \& CAMPBELL, M. 2011. Respectful Management of Serious Clinical Adverse Events. IHI Innovation Series White Paper. Second Edition ed. Cambridge, Massachusetts: Institute for Healthcare Improvement.

CRAVENS, C. \& EARP, J. A. 2009. Disclosure and apology: patient-centered approaches to the public health problem of medical error. North Carolina Medical Journal, 70, 140-146.

CRIGGER, N. J. 2004. Always having to say you're sorry: an ethical response to making mistakes in professional practice. Nursing Ethics, 11, 568-576.

DAUER, E. A. 2011. Medical injury, patients' claims and the effects of government responses in Anglo-American legal systems. British Medical Journal Quality \& Safety, 20, 735-737.

DE CASSIA PIRES COLI, R., DOS ANJOS, M. F. \& PEREIRA, L. L. 2010. The attitudes of nurses from an intensive care unit in the face of errors: an approach in light of bioethics. Revista Latino Americana Enfermagem, 18, 324-330.

DE FREITAS, G. F., HOGA, L. A., DE FA'TIMA PRADO FERNANDES, M., SILES GONZA LEZ, J., SOLANO RUIZ, M. C. \& BONINI, B. B. 2011. Brazilian registered nurses' perceptions and attitudes towards adverse events in nursing care: a phenomenological study. Journal of Nursing Management, 19, 331-338. 
DEPARTMENT OF VETERANS AFFAIRS 2008 Disclosure of Adverse Events to Patients: VHA directive 2008-022. Washington DC: United States Veterans Health Administration available at:( http://www.ethics.va.gov/docs/policy/VHA_Directive_2008002_Disclosure_of_Adverse_Events_20080118.pdf, accessed 21 August 2012).

DINTZIS, S. M., STETSENKO, G. Y., SITLANI, C. M., GRONOWSKI, A. M., ASTION, M. L. \& GALlAGHER, T. H. 2011. Communicating pathology and laboratory errors: Anatomic pathologists' and laboratory medical directors' attitudes and experiences. American Journal of Clinical Pathology, $135,760-765$.

DOODY, O., SLEVIN, E. \& TAGGART, L. 2013. Focus group interviews part 3: analysis. British Journal Nursing, 22, 266-269.

DOWLING, M,. 2007. From Husserl to van Manen. A review of different phenomenological approaches. International Journal of Nursing Studies, 44, $131-142$

EAVES-LEANOS, A. \& DUNN, E. J. 2012. Open disclosure of adverse events: transparency and safety in health care. Surgical Clinics of North America, 92, 163-177.

EDREES, H. H., PAINE, L. A., FEROLI, E. R. \& WU, A. W. 2011. Health care workers as second victims of medical errors. Polish Archives Internal Medicine, 121, 101-108.

ESPIN, S., LEVINSON, W., REGEHR, G., BAKER, G. R. \& LINGARD, L. 2006. Error or "act of God"? A study of patients' and operating room team members' perceptions of error definition, reporting, and disclosure. Surgery, 139, 6-14.

EVERETT, J. P., WALTERS, C. A., STOTTlEMYER, D. L., KNIGHT, C. A., OPPENBERG, A. A. \& ORR, R. D. 2011. To lie or not to lie: resident physician attitudes about the use of deception in clinical practice. Journal of Medical Ethics, 37, 333-338.

FALLOWFIELD, L. \& JENKINS, V. 2004. Communicating sad, bad, and difficult news in medicine. Lancet, 363, 312-319.

FEIN, S., HILBORNE, L., KAGAWA-SINGER, M. \& SPIRITUS, E. 2005. A Conceptual Model for Disclosure of Medical Errors. Advances in Patient Safety from Research to Implementation, 2, 483-494

FEIN, S. P., HILBORNE, L. H., SPIRITUS, E. M., SEYMANN, G. B., KEENAN, C. R., SHOJANIA, K. G., KAGAWA-SINGER, M. \& WENGER, N. S. 2007. 
The many faces of error disclosure: a common set of elements and a definition. Journal General Internal Medicine, 22, 755-761.

GALLAGHER, T. H., DENHAM, C. R., LEAPE, L. L., AMORI, G. \& LEVINSON, W. 2007a. Disclosing Unanticipated Outcomes to Patients: The Art and Practice. Journal of Patient Safety, 3, 158-165.

GALlAGHER, T. H., GARBUTT, J. M., WATERMAN, A. D., FlUM, D. R., LARSON, E. B., WATERMAN, B. M., DUNAGAN, W. C., FRASER, V. J. \& LEVINSON, W. 2006a. Choosing your words carefully: how physicians would disclose harmful medical errors to patients. Archives of Internal Medicine, 166, 1585-1593.

GALLAGHER, T. H. \& LEVINSON, W. 2005. Disclosing harmful medical errors to patients: a time for professional action. Archives of Internal Medicine, 165, 1819-1824.

GALlAGHER, T. H., STUDDERT, D. \& LEVINSON, W. 2007b. Disclosing harmful medical errors to patients. New England Journal of Medicine, 356, 2713-2719.

GALlAGHER, T. H., WATERMAN, A. D., EBERS, A. G., FRASER, V. J. \& LEVINSON, W. 2003. Patients' and physicians' attitudes regarding the disclosure of medical errors. Journal American Medical Association, 289, 1001-1007.

GALlAGHER, T. H., WATERMAN, A. D., GARBUTT, J. M., KAPP, J. M., CHAN, D. K., DUNAGAN, W. C., FRASER, V. J. \& LEVINSON, W. 2006b. US and Canadian physicians' attitudes and experiences regarding disclosing errors to patients. Archives of Internal Medicine, 166, 1605-1611.

GARBUTT, J., WATERMAN, A. D., KAPP, J. M., DUNAGAN, W. C., LEVINSON, W., FRASER, V. \& GALLAGHER, T. H. 2008. Lost opportunities: how physicians communicate about medical errors. Health Affairs (Millwood), 27, 246-55.

GHALANDARPOORATTAR, S. M., KAVIANI, A. \& ASGHARI, F. 2012. Medical error disclosure: the gap between attitude and practice. Postgraduate Medical Journal, 88, 130-133.

GIORGI, A 2005. The phenomenological movement and research in the human sciences. Nursing Science Quaterly, 21, 379-398.

GREENE, D. A. 2009. Nurses' experiences with the disclosure of errors to patients. Ph.D., Georgia State University. 
GUNDERSON, A. J., SMITH, K. M., MAYER, D. B., MCDONALD, T. \& CENTOMANI, N. 2009. Teaching medical students the art of medical error full disclosure: evaluation of a new curriculum. Teaching \& Learning in Medicine, 21, 229-232.

HANNAWA, A. F., BECHER, H., MAZOR, K., PAUL, N. \& RAMSEY, J. 2013. Building bridges: future directions for medial error disclosure research. Patient Education \& Counseling, 1-9.

HANNES, K., AERTGEERTS, B., SCHEPERS, R., GOEDHUYS, J. \& BUNTINX, F. 2005. Evidence-based medicine: a discussion of the most frequently occurring criticisms. Ned Tijdschr Geneeskd, 149, 1983-1988.

HANNES, K. \& LOCKWOOD, C. 2011. Pragmatism as the philosophical foundation for the Joanna Briggs meta aggregative approach to qualitative evidence synthesis. Journal of Advanced Nursing, 67, 1632-1642.

HARVARD HOSPITALS, 2006. When Things go Wrong, Responding to Adverse Events, A Consenses Statement of the Harvand Hospitals. Massachusetts, Massachusetts Coalition for the Prevention of Medical Errors: 37. (available http://www.ihi.org/knowledge/Pages/Publications/WhenThingsGoWrongResp ondingtoAdverseEvents.aspx, accessed 25 Nov 2012).

HEBERT, P. C., LEVIN, A. V. \& ROBERTSON, G. 2001. Bioethics for clinicians: 23. Disclosure of medical error. Canadian Medical Association Journal, 164, 509-513.

HEVIA, A. \& HOBGOOD, C. 2003. Medical error during residency: to tell or not to tell. Annual Emergency Medicine, 42, 565-570.

HINGORANI, M., WONG, T. \& VAFIDIS, G. 1999. Attitudes after unintended injury during treatment a survey of doctors and patients. West Journal Medicine, 171, 81-82.

IEDEMA, R., ALLEN, S., BRITTON, K., PIPER, D., BAKER, A., GRBICH, C., ALLAN, A., JONES, L., TUCKETT, A., WILLIAMS, A., MANIAS, E. \& GALLAGHER, T. H. 2011a. Patients' and family members' views on how clinicians enact and how they should enact incident disclosure: the "100 patient stories" qualitative study. British Medical Journal, 343, 1-9.

IEDEMA, R., ALLEN, S., SORENSEN, R. \& GALLAGHER, T. 2011b. What prevents incident disclosure, and what can be done to promote it? Joint Commission Journal on Quality \& Patient Safety, 37, 409-417.

IEDEMA, R., MALLOCK, N., SORENSEN, R., MANIAS, E., TUCKETT, A. \& WILLIAMS, A. 2007. Final Report: Evaluation of the Pilot of the National 
Open Disclosure Standard. Sydney: Australian Commission of Safety and Quality in Health Care, 1-161.

KAISER FAMILY FOUNDATION, 2005. National Survey on consumers' experience with patient safety and quality information.[available; http://www.kfforg/kaiserpolls/7210.cfm. accessed 1 May 2012]

KALDJIAN, L., JONES, E. W. \& ROSENTHAL, G. E. 2006a. An Empirically Derived Taxonomy of Factors Affecting Physicians' Willingness to Disclose Medical Errors. Journal General Internal Medicine, 21, 942-948.

KALDJIAN, L., JONES, E. W. \& Wu, B. J. 2006b. Disclosing Medical Errors to Patients: Attitudes and Practices of Physicians and Trainees. Journal General Internal Medicine, 22, 988-996.

KALRA, J. 2004. Medical errors: overcoming the challenges. Clinical Biochemistry. $37,1063-1071$

KINN, L. G. HOLGERSEN,H. \& EKELAND, T. 2013. Metasynthesis and Bricolage: An Artistic Exercise of Creating a Collage of Meaning. Quality Health Research, 23, 9. 1285-1292.

$\mathrm{KOCH}, \mathrm{T} .1999$. An interpretative research process: revisiting phenomenological and hermeneutical approaches. Nurse Researcher, 6,3. 20-24.

KOHN, L., CORRIGAN, J. M. \& DONALDSON, M. 2000. To Err is Human Building a Safer Health System. A report of the Committee on Healthcare in America. In: PRESS, N. A. (ed.). Washington DC: Institute of Medicine.

LAMB, R. M., STUDDERT, D. M., BOHMER, R. M., BERWICK, D. M. \& BRENNAN, T. A. 2003. Hospital disclosure practices: results of a national survey. Health Affairs (Millwood), 22, 73-83.

LEAPE, L. 2006. Full Disclosure and Apology: an idea whose time has come. The Physician Executive, March-April, 16-18.

LEVINSON, W. 2009. Disclosing medical errors to patients: A challenge for health care professionals and institutions. Patient Education Counselling.76, 296299.

LINTHORST, G. E., KALLIMANIS-KING, B. L., DOUWES DEKKER, I., HOEKSTRA, J. B. \& DE HAES, J. C. 2012. What contributes to internists' willingness to disclose medical errors? Netherland Journal Medicine, 70, 242248. 
LOPEZ, L., WEISSMAN, J. S., SCHNEIDER, E. C., WEINGART, S. N., COHEN, A. P. \& EPSTEIN, A. M. 2009. Disclosure of hospital adverse events and its association with patients' ratings of the quality of care. Archives of Internal Medicine, 169, 1888-1894.

LOREN, D. J., KLEIN, E. J., GARBUTT, J., KRAUSS, M. J., FRASER, V., DUNAGAN, W. C., BROWNSTEIN, D. R. \& GALLAGHER, T. H. 2008. Medical error disclosure among pediatricians: Choosing carefully what we might say to parents. Archives of Pediatrics and Adolescent Medicine, 162, 922-927.

LUCE, J. M. 2006. Acknowledging our mistakes. Critical Care Medicine, 34, 15751576.

MACDONALD, N. \& ATTARAN, A. 2009. Medical errors, apologies and apology laws. Canadian Medical Association Journal, 180, 11-13.

MADDEN, B. \& COCKBURN, T. 2007. Bundaberg and beyond: duty to disclose adverse events to patients. Journal Law Medicine, 14, 501-527.

MANEN, M. V., 1990. Hermeneutic Phenomenological Writing. In Researching Lived Experience: human science for an action sensitive pedagogy, Ontario: The University of Western Ontario, 111-133.

MANSER, T. 2011. Managing the aftermath of critical incidents: meeting the needs of health-care providers and patients. Best Practice Research Clinical Anaesthesiology, 25, 169-179.

MANSER, T. \& STAENDER, S. 2005. Aftermath of an adverse event: supporting health care professionals to meet patient expectations through open disclosure. Acta Anaesthesiology Scandinavia, 49, 728-734.

MARINER, W. K. 2001. Medical error reporting: professional tension between confidentiality and liablity. Issue Brief, Mass Health Policy Forum, 13, 1-35.

MATLOW, A., STEVENS, P., HARRISON, C. \& LAXER, R. M. 2006. Disclosure of medical errors. Pediatric Clinics North America, 53, 1091-1104.

MAZOR, K. M., ROBLIN, D. W., GREENE, S. M., LEMAY, C. A., FIRNENO, C. L., CALVI, J., PROUTY, C. D., HORNER, K. \& GALlAGHER, T. H. 2012. Toward patient-centered cancer care: patient perceptions of problematic events, impact, and response. Journal Clinical Oncology, 30, 1784-1790.

MOSKOP, J. C., GEIDERMAN, J. M., HOBGOOD, C. D. \& LARKIN, G. L. 2006. Emergency Physicians and Disclosure of Medical Errors. Annals of Emergency Medicine, 48, 523-531. 
NATIONAL QUALITY FORUM (NQF), 2010. Safe Practices for Better Healthcare Update: A Consensus Report. Washington, DC: NQF (available https://www.qualityforum.org/2010/Safe_Practice_10_Abridge. accessed 21 March 2012).

O'CONNOR, E., COATES, H. M., YARDLEY, I. E. \& WU, A. W. 2010. Disclosure of patient safety incidents: a comprehensive review. International Journal for Quality in Health Care, 22, 371-379.

PEARSON, A. 2004. Balancing the eidence: incoperating the syntheis of qualitaive data into systematic reviews. JBI reports, 2, 45-46.

PEARSON, A., JORDAN, Z. 2007. Evidence Based Clinical Practice in Nursing and Health Care Assimilating Research, Experience and Expertise. Hong Kong: Blackwell Publishing.

PEARSON, A., WIECHULA, R., COURT, A. \& LOCKWOOD, C. 2005. The JBI model of evidence-based healthcare. International Journal Evidence Based Healthcare, 3, 207-215.

PETRONIO, S., TORKE, A., BOSSLET, G., ISENBERG, S., WOCIAL, L. \& HELFT, P. 2013. Disclosing Medical Mistakes: A Communication Management Plan for Physicians. The Permanente Journal, 17, 73-79.

PIPER, D. \& IEDEMA, R. 2011. Literature review: incident disclosure research, policy and legal reforms since 2008 Centre for Health Communication (University of Technology Sydney), and Australian Commission on Safety and Quality in Health, 1-62.

RICHMAN, J., MASON, T., MASON-WHITEHEAD, E., MCINTOSH, A. \& MERCER, D. 2009. Social aspects of clinical errors. International Journal of Nursing Studies, 46, 1148-1155.

ROWE, M. 2004. Doctors' responses to medical errors. Critical Review Oncology Hematolology, 52, 147-63.

SCHWAPPACH, D. L. \& KOECK, C. M. 2004. What makes an error unacceptable? A factorial survey on the disclosure of medical errors. Internal Journal Quality Health Care, 16, 317-326.

SHANNON, S. E., FOGLIA, M. B., HARDY, M. \& GALLAGHER, T. H. 2009. Disclosing errors to patients: perspectives of registered nurses. Joint Commission Journal on Quality \& Patient Safety, 35, 5-12. 
SMITH, M. L. \& FORSTER, H. P. 2000. Morally managing medical mistakes. Cambridge Quarterly of Healthcare Ethics, 9, 38-53.

SORENSEN, R., IEDEMA, R., PIPER, D., MANIAS, E., WILLIAMS, A. \& TUCKETT, A. 2008. Health care professionals' views of implementing a policy of open disclosure of errors. Journal of Health Services Research and Policy, 13, 227-232.

SORENSEN, R., IEDEMA, R., PIPER, D., MANIAS, E., WILLIAMS, A. \& TUCKETT, A. 2010. Disclosing clinical adverse events to patients: can practice inform policy? Health Expectations, 13, 148-159.

STUDDERT, D. M., PIPER, D. \& IEDEMA, R. 2010. Legal aspects of open disclosure II: attitudes of health professionals findings from a national survey. Medical Journal Australia, 193, 351-355.

STUDDERT, D. M. \& RICHARDSON, M. W. 2010. Legal Aspects of Open Disclosure: a review of Australian law. Medical Journal Australia, 193, 5, 273-276.

THE JOANNA BRIGGS INSTITUTE. 2011. Joanna Briggs Institute Reviewers Manual, The Joanna Briggs Institute.

THORNE, S. 2010. Data analysis in qualitaive research. Evidence Based Nursing, 3, $68-70$.

THORNE, S., JENSEN, L., KEARNEY, M. H., NOBLIT, G. \& SANDELOWSKI, M. 2004. Qualitative metasynthesis: reflections on methodological orientation and ideological agenda. Quality Health Research, 14, 1342-1365.

VENUS, E., GALAM, E., AUBERT, J. P. \& NOUGAIREDE, M. 2012. Medical errors reported by French general practitioners in training: results of a survey and individual interviews. British Medical Journal Quality \& Safety, 21, 279 286.

VINCENT, J. L. 1998. Information in the ICU: are we being honest with our patients? The results of a European questionnaire. Intensive Care Medicine, 24, 1251-1256.

WAGNER, L. M., HARKNESS, K., HEBERT, P. C. \& GALLAGHER, T. H. 2012. Nurses' perceptions of error reporting and disclosure in nursing homes. Journal Nursing Care Quality, 27, 63-69.

WATERMAN, A. D., GARBUTT, J., HAZEL, E., DUNAGAN, W. C., LEVINSON, W., FRASER, V. J., GALlAGHER, T. H. 2007. The emotional impact of 
medical errors on practicing physicians in the United States and Canada. Joint Commission Journal of Quality \& Patient Safety, 33,8,467-476.

WAITE, M. 2005. To tell the truth: the ethical and legal implications of disclosure of medical error. Health Law Journal, 13, 1-33.

WALSH D., \& DOWNE. 2005. Meta-synthesis method for qualitative research: a literature review. Journal of Advanced Nursing, 50, 204-211.

WEISSMAN, J. S., ANNAS, C. L., EPSTEIN, A. M., SCHNEIDER, E. C., CLARRIDGE, B., KIRLE, L., GATSONIS, C., FEIBELMANN, S. \& RIDLEY, N. 2005. Error Reporting and Disclosure Systems: views from hospital leaders. Journal of the American Medical Association, 293, 13591366.

WHITE, A. A., GALlAGHER, T. H., KRAUSS, M. J., GARBUTT, J., WATERMAN, A. D., DUNAGAN, W. C., FRASER, V. J., LEVINSON, W. \& LARSON, E. B. 2008. The attitudes and experiences of trainees regarding disclosing medical errors to patients. Academic Medicine, 83, 250-256.

WINSLADE, W. \& MCKINNEY, E. B. 2006. To tell or not to tell: disclosing medical error. Journal of Law, Medicine \& Ethics, 34, 813-816.

WITMAN, A. B., PARK, D. M. \& HARDIN, S. B. 1996. How do patients want physicians to handle mistakes? A survey of internal medicine patients in an academic setting. Archives of Internal Medicine, 156, 2565-2569.

WU, A., FOLKMAN, S., MACPHEE, S. \& LO, B. 1993. How do house officers cope with their mistakes. Western Journal Medicine, 159, 565-569.

WU, A. W. 2000. Medical error: the second victim. The doctor who makes the mistake needs help too. British Medical Journal, 320, 726-727.

WU, A. W., FOLKMAN, S., MCPHEE, S. J. \& LO, B. 1991. Do house officers learn from their mistakes? Journal American Medical Association, 265, 2089-2094. 


\section{Appendix I: Detailed Database Search Strategy}

PubMed searched 10/09/2012

\begin{tabular}{|c|c|c|}
\hline Search Number & Search term & Number of citations \\
\hline 1 & medical error[mh] & 76,106 \\
\hline 2 & medical error*[tiab] & 2,639 \\
\hline 3 & mistake* $*$ tiab] & 14,062 \\
\hline 4 & surgical error*[tiab] & 252 \\
\hline 5 & diagnostic error*[tiab] & 2,515 \\
\hline 6 & therapeutic error*[tiab] & 214 \\
\hline 7 & treatment error*[tiab] & 236 \\
\hline 8 & iatrogenic diseases[mh] & 12,143 \\
\hline 9 & iatrogenic [tiab] & 19,093 \\
\hline 10 & medical incident*[tiab] & 97 \\
\hline 11 & adverse event $*[$ tiab] & 63,324 \\
\hline 12 & health care error* [tiab] & 37 \\
\hline 13 & healthcare error*[tiab] & 16 \\
\hline 14 & physician error*[tiab] & 68 \\
\hline 15 & nurse's error*[tiab] & 294 \\
\hline 16 & nursing error*[tiab] & 50 \\
\hline 17 & patient safety[mh] & 1,315 \\
\hline 18 & patient safety[tiab] & 11,467 \\
\hline 19 & patients safety[tiab] & 82 \\
\hline 20 & patient's safety[tiab] & 181 \\
\hline 21 & malpractice[mh:noexp] & 24,383 \\
\hline 22 & malpractice [tiab] & 7,932 \\
\hline 23 & $\begin{array}{l}1 \text { OR } 2 \text { OR } 3 \text { OR } 4 \text { OR } 5 \text { OR } \\
6 \text { OR } 7 \text { OR } 8 \text { OR } 9 \text { OR } 10 \text { OR } \\
11 \text { OR } 12 \text { OR } 13 \text { OR } 14 \text { OR } \\
15 \text { OR } 16 \text { OR } 17 \text { OR } 18 \text { OR } \\
19 \text { OR } 20 \text { OR } 21 \text { OR } 22\end{array}$ & 197,140 \\
\hline 24 & truth disclosure[mh:noexp] & 10,795 \\
\hline 25 & truth[tiab] & 6,339 \\
\hline 26 & disclosure[mh:noexp] & 9,785 \\
\hline 27 & disclos*[tiab] & 47,310 \\
\hline 28 & ethics,clinical[mh] & 56,663 \\
\hline 29 & ethics[sh] & 42,271 \\
\hline 30 & ethic $*[$ tiab $]$ & 72,645 \\
\hline 31 & deception[mh:noexp] & 3,131 \\
\hline 32 & deception[tiab] & 1,412 \\
\hline 33 & honest $*[$ tiab $]$ & 3,101 \\
\hline 34 & dishonest*[tiab] & 393 \\
\hline 35 & 24 OR 25 OR 26 OR 27 OR & 193,801 \\
\hline
\end{tabular}




\begin{tabular}{|l|l|l|}
\hline & $\begin{array}{l}\text { 28 OR 29 OR 30 OR 31 OR } \\
\text { 32 OR 33 OR 34 }\end{array}$ & \\
\hline 36 & 23 AND 35 & 4005 \\
\hline
\end{tabular}

CINAHL searched 15/09/2012

\begin{tabular}{|c|c|c|}
\hline Search Number & Search term & Number of citations \\
\hline 1 & MH "health care error+" & 20,916 \\
\hline 2 & TI "health care error*” & 14 \\
\hline 3 & $A B$ "heath care error*" & 24 \\
\hline 4 & TI "healthcare error*" & 5 \\
\hline 5 & $\mathrm{AB}$ "healthcare error*" & 10 \\
\hline 6 & TI “medical error*” & 850 \\
\hline 7 & $\mathrm{AB}$ "medical error*" & 737 \\
\hline 8 & TI "mistake*" & 1,010 \\
\hline 9 & AB "mistake*" & 1,530 \\
\hline 10 & TI "surgical error*" & 28 \\
\hline 11 & AB "surgical error*" & 14 \\
\hline 12 & MH "diagnostic errors" & 4,330 \\
\hline 13 & TI "diagnostic error*" & 107 \\
\hline 14 & $\mathrm{AB}$ "diagnostic error*” & 66 \\
\hline 15 & TI "therapeutic error*" & 6 \\
\hline 16 & $\mathrm{AB}$ "therapeutic error*" & 17 \\
\hline 17 & MH "treatment error*" & 4,205 \\
\hline 18 & TI "treatment error*" & 6 \\
\hline 19 & $\mathrm{AB}$ "treatment error*" & 33 \\
\hline 20 & MH "iatrogenic disease" & 1,189 \\
\hline 21 & TI "iatrogenic" & 520 \\
\hline 22 & $\mathrm{AB}$ "iatrogenic" & 1,235 \\
\hline 23 & TI "medical incident*" & 8 \\
\hline 24 & AB "medical incident*" & 23 \\
\hline 25 & MH "adverse health care event" & 2,367 \\
\hline 26 & TI "adverse event*” & 1390 \\
\hline 27 & $\mathrm{AB}$ "adverse event*" & 10,963 \\
\hline 28 & TI "physician error*" & 2 \\
\hline 29 & $\mathrm{AB}$ "physician error*" & 5 \\
\hline 30 & TI "nurse's error*" & 4 \\
\hline 31 & $\mathrm{AB}$ "nurse's error*” & 3 \\
\hline 32 & TI "nursing error*” & 27 \\
\hline 33 & $\mathrm{AB}$ "nursing error*” & 34 \\
\hline 34 & MH "patient safety" & 22,007 \\
\hline 35 & TI "patient safety" & 4,747 \\
\hline 36 & $\mathrm{AB}$ "patient safety" & 3,992 \\
\hline 37 & TI "patients safety" & 43 \\
\hline 38 & AB "patients safety" & 81 \\
\hline
\end{tabular}




\begin{tabular}{|c|c|c|}
\hline 39 & TI "patient's safety" & 5 \\
\hline 40 & AB "patient's safety" & 59 \\
\hline 41 & MH "malpractice" & 6,235 \\
\hline 42 & TI "malpractice" & 1,754 \\
\hline 43 & $\mathrm{AB}$ "malpractice" & 885 \\
\hline 44 & $\begin{array}{l}1 \text { OR } 2 \text { OR } 3 \text { OR } 4 \text { OR } 5 \text { OR } 6 \\
\text { OR } 7 \text { OR } 8 \text { OR } 9 \text { OR } 10 \text { OR } 11 \\
\text { OR } 12 \text { OR } 13 \text { OR } 14 \text { OR } 15 \text { OR } \\
16 \text { OR } 17 \text { OR } 18 \text { OR } 19 \text { OR } 20 \\
\text { OR } 21 \text { OR } 22 \text { OR } 23 \text { OR } 24 \text { OR } \\
25 \text { OR } 26 \text { OR } 27 \text { OR } 28 \text { OR } 29 \\
\text { OR } 30 \text { OR } 31 \text { OR } 32 \text { OR } 33 \text { OR } \\
34 \text { OR } 35 \text { OR } 36 \text { OR } 37 \text { OR } 38 \\
\text { OR } 39 \text { OR } 40 \text { OR } 41\end{array}$ & 64,106 \\
\hline 45 & MH "truth disclosure" & 5,629 \\
\hline 46 & TI "truth" & 1,550 \\
\hline 47 & $\mathrm{AB}$ "truth" & 876 \\
\hline 48 & TI "disclos*” & 2,169 \\
\hline 49 & AB "disclos*" & 4,430 \\
\hline 50 & MH "ethics,professional+" & 13,809 \\
\hline 51 & TI "ethic*” & 16,639 \\
\hline 52 & AB "ethic*” & 14,473 \\
\hline 53 & MH “deception” & 944 \\
\hline 54 & IT “deception" & 156 \\
\hline 55 & $\mathrm{AB}$ "deception" & 179 \\
\hline 56 & MH "honesty" & 663 \\
\hline 57 & IT "honest*" & 325 \\
\hline 58 & AB "honest*" & 769 \\
\hline 59 & TI "dishonest*” & 79 \\
\hline 60 & AB "dishonest*" & 90 \\
\hline 61 & $\begin{array}{l}45 \text { OR } 46 \text { OR } 47 \text { OR } 48 \text { OR } 49 \\
\text { OR } 50 \text { OR } 51 \text { OR } 52 \text { OR } 53 \text { OR } \\
54 \text { OR } 55 \text { OR } 56 \text { OR } 57 \text { OR } 58 \\
\text { OR } 59 \text { OR } 60\end{array}$ & 45,725 \\
\hline 62 & 44 AND 61 & 1,899 \\
\hline
\end{tabular}

PsycINFO: Searched 15/09/2012

\begin{tabular}{|l|l|l|}
\hline Search Number & Search term & Number of citations \\
\hline 1 & DE "errors" & 7,345 \\
\hline 2 & SU "medical" & 60,941 \\
\hline 3 & SU "nurs*" & 38,874 \\
\hline 4 & SU "physician" & 5,510 \\
\hline 5 & SU "surgical & 4,383 \\
\hline 6 & 2 OR 3 OR 4 OR 5 & 104,754 \\
\hline
\end{tabular}




\begin{tabular}{|c|c|c|}
\hline 7 & 1 AND 6 & 379 \\
\hline 8 & TI "medical error"” & 88 \\
\hline 9 & AB "medical error*" & 345 \\
\hline 10 & TI "mistake*” & 537 \\
\hline 11 & AB "mistake*" & 7,229 \\
\hline 12 & TI "surgical error*” & 1 \\
\hline 13 & AB "surgical error*" & 11 \\
\hline 14 & TI "diagnostic error*" & 49 \\
\hline 15 & AB "diagnostic error*” & 196 \\
\hline 16 & TI "therapeutic error*” & 3 \\
\hline 17 & AB "therapeutic error*" & 32 \\
\hline 18 & TI "treatment error*" & 4 \\
\hline 19 & $\mathrm{AB}$ "treatment error*" & 27 \\
\hline 20 & IT "iatrogenic" & 202 \\
\hline 21 & $\mathrm{AB}$ "iatrogenic" & 1,086 \\
\hline 22 & TI "medical incident*" & 2 \\
\hline 23 & AB "medical incident*" & 5 \\
\hline 24 & TI "adverse event*” & 315 \\
\hline 25 & AB "adverse event*” & 6,008 \\
\hline 26 & AB "health care error*" & 5 \\
\hline 27 & TI "healthcare error*" & 32 \\
\hline 28 & AB "healthcare error*” & 7 \\
\hline 29 & TI "health care error*" & 1 \\
\hline 30 & TI "physician error*" & 28 \\
\hline 31 & AB "physician error*” & 8 \\
\hline 32 & TI "nurse's error*” & 9 \\
\hline 33 & AB "nurse's error*” & 18 \\
\hline 34 & TI "nursing error*" & 2 \\
\hline 35 & $\mathrm{AB}$ "nursing error*" & 9 \\
\hline 36 & TI "patient safety" & 321 \\
\hline 37 & AB "patient safety" & 1,083 \\
\hline 38 & TI "patients safety" & 4 \\
\hline 39 & AB "patients safety" & 42 \\
\hline 40 & TI “patient's safety" & 19 \\
\hline 41 & AB "patient's safety" & 21 \\
\hline 42 & DE "professional liability" & 1,766 \\
\hline 43 & TI "malpractice" & 317 \\
\hline 44 & AB "malpractice" & 1,102 \\
\hline 45 & $\begin{array}{l}7 \text { OR } 8 \text { OR } 9 \text { OR } 10 \text { OR } 11 \text { OR } \\
12 \text { OR } 13 \text { OR } 14 \text { OR } 15 \text { OR } 16 \\
\text { OR } 17 \text { OR } 18 \text { OR } 19 \text { OR } 20 \text { OR } \\
21 \text { OR } 22 \text { OR } 23 \text { OR } 24 \text { OR } 25 \\
\text { OR } 26 \text { OR } 27 \text { OR } 28 \text { OR } 29 \text { OR } \\
30 \text { OR } 31 \text { OR } 32 \text { OR } 33 \text { OR } 34 \\
\text { OR } 35 \text { OR } 36 \text { OR } 37 \text { OR } 38 \text { OR }\end{array}$ & 16,460 \\
\hline
\end{tabular}




\begin{tabular}{|c|c|c|}
\hline & $\begin{array}{l}39 \text { OR } 40 \text { OR } 41 \text { OR } 42 \text { OR } 43 \\
\text { OR } 44\end{array}$ & \\
\hline 46 & DE "truth" & 1,125 \\
\hline 47 & TI "truth" & 1,872 \\
\hline 48 & AB "truth" & 10,136 \\
\hline 49 & TI "disclos*” & 4,531 \\
\hline 50 & AB "disclos*" & 15,777 \\
\hline 51 & DE "ethic*" & 9,550 \\
\hline 52 & TI "ethic*" & 15,679 \\
\hline 53 & AB "ethic*" & 43,176 \\
\hline 54 & DE "deception" & 4,314 \\
\hline 55 & IT “deception" & 1,634 \\
\hline 56 & AB "deception" & 3,524 \\
\hline 57 & DE "honesty" & 893 \\
\hline 58 & IT "honest*" & 522 \\
\hline 59 & AB "honest*" & 5,434 \\
\hline 60 & TI "dishonest*" & 207 \\
\hline 61 & AB "dishonest*" & 870 \\
\hline 62 & $\begin{array}{l}46 \text { OR } 47 \text { OR } 48 \text { OR } 49 \text { OR } 50 \\
\text { OR } 51 \text { OR } 52 \text { OR } 53 \text { OR } 54 \text { OR } \\
55 \text { OR } 56 \text { OR } 57 \text { OR } 58 \text { OR } 59 \\
\text { OR } 60 \text { OR } 61\end{array}$ & 82,616 \\
\hline 63 & 45 AND 62 & 698 \\
\hline
\end{tabular}

Embase searched on 10/09/2012

\begin{tabular}{|l|l|l|}
\hline Search Number & Search term & Number of citations \\
\hline 1 & 'medical error'/exp & 73,714 \\
\hline 2 & 'medical error':ab,ti & 954 \\
\hline 3 & 'medical errors':ab,ti & 2,380 \\
\hline 4 & mistake*:ab,ti & 18,235 \\
\hline 5 & 'surgical error':ab,ti & 105 \\
\hline 6 & 'surgical error':ab,ti & 164 \\
\hline 7 & 'diagnostic error':ab,ti & 995 \\
\hline 8 & 'diagnostic errors':ab,ti & 2,191 \\
\hline 9 & 'therapeutic error':ab,ti & 80 \\
\hline 10 & 'therapeutic errors':ab,ti & 237 \\
\hline 11 & 'treatment error':ab,ti & 85 \\
\hline 12 & 'treatment errors':ab,ti & 245 \\
\hline 13 & 'iatrogenic disease'/de & 20,984 \\
\hline 14 & iatrogenic: ab,ti & 24,931 \\
\hline 15 & 'medical incident':ab,ti & 51 \\
\hline 16 & 'medical incidents':ab,ti & 68 \\
\hline 17 & 'adverse event':ab,ti & 15,852 \\
\hline
\end{tabular}




\begin{tabular}{|c|c|c|}
\hline 18 & 'adverse events':ab,ti & 82,520 \\
\hline 19 & 'health care error': ab,ti & 10 \\
\hline 20 & 'health care errors':ab,ti & 31 \\
\hline 21 & 'healthcare errors': ab,ti & 13 \\
\hline 22 & 'physician error':ab,ti & 48 \\
\hline 23 & 'physician errors':ab,ti & 30 \\
\hline 24 & 'nurses error':ab,ti & 5 \\
\hline 25 & 'nurses errors':ab,ti & 10 \\
\hline 26 & 'nursing error':ab,ti & 19 \\
\hline 27 & 'nursing errors':ab,ti & 38 \\
\hline 28 & 'patient safety'/de & 35,860 \\
\hline 29 & 'patient safety':ab,ti & 14,107 \\
\hline 30 & 'patients safety':ab,ti & 623 \\
\hline 31 & 'malpractice'/de & 29,418 \\
\hline 32 & 'malpractice':ab,ti & 8,796 \\
\hline 33 & $\begin{array}{l}1 \text { OR } 2 \text { OR } 3 \text { OR } 4 \text { OR } 5 \text { OR } 6 \\
\text { OR } 7 \text { OR } 8 \text { OR } 9 \text { OR } 10 \text { OR } 11 \\
\text { OR } 12 \text { OR } 13 \text { OR } 14 \text { OR } 15 \text { OR } \\
16 \text { OR } 17 \text { OR } 18 \text { OR } 19 \text { OR } 20 \\
\text { OR } 21 \text { OR } 22 \text { OR } 23 \text { OR } 24 \text { OR } \\
25 \text { OR } 26 \text { OR } 27 \text { OR } 28 \text { OR } 29 \\
\text { OR } 30 \text { OR } 31 \text { OR } 32\end{array}$ & 284,009 \\
\hline 34 & 'truth':ad,ti & 8,796 \\
\hline 35 & disclos*:ab,ti & 57,787 \\
\hline 36 & $\begin{array}{l}\text { 'interpersonal } \\
\text { communication':de }\end{array}$ & 100,347 \\
\hline 37 & 'medical ethics':de & 87,531 \\
\hline 38 & ethic*:ab,ti & 91,944 \\
\hline 39 & 'deception':de & 518 \\
\hline 40 & ‘deception':ad,ti & 1,756 \\
\hline 41 & 'honesty':de & 381 \\
\hline 42 & honest*:ab,ti & 3,657 \\
\hline 43 & dishonest*:ab,ti & 447 \\
\hline 44 & $\begin{array}{l}34 \text { OR } 35 \text { OR } 36 \text { OR } 37 \text { OR } 38 \\
\text { OR } 39 \text { OR } 40 \text { OR } 41 \text { OR } 42 \text { OR } \\
43\end{array}$ & 122,840 \\
\hline 45 & 33 AND 44 & 2,036 \\
\hline
\end{tabular}




\section{Appendix II: QARI Appraisal instrument}

\section{JBI QARI Critical Appraisal Checklist for Interpretive \& Critical Research}

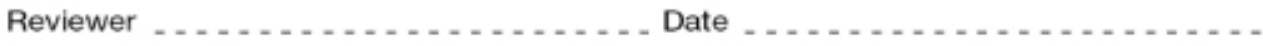

Author Year Record Number _

1. Is there congruity between the stated philosophical perspective and the research methodology?

2 . Is there congruity between the research methodology and the research question or objectives?

3. Is there congruity between the research methodology and the methods used to collect data?

4. Is there congruity between the research methodology and the representation and analysis of data?

5. Is there congruity between the research methodology and the interpretation of results?

6. Is there a statement locating the researcher culturally or theoretically?

7. Is the influence of the researcher on the research, and vice- versa, addressed?

8. Are participants, and their voices, adequately represented?

9. Is the research ethical according to current criteria or, for recent studies, and is there evidence of ethical approval by an appropriate body?

10. Do the conclusions drawn in the research report flow from the analysis, or interpretation, of the data?

Overall appraisal:

Include

Exclude

No Unclear Not Applicable

Yes

Unclear

Comments (Including reason for exclusion) 
Appendix III: QARI Data extraction instrument

\section{JBI QARI Data Extraction Form for Interpretive \& Critical Research}

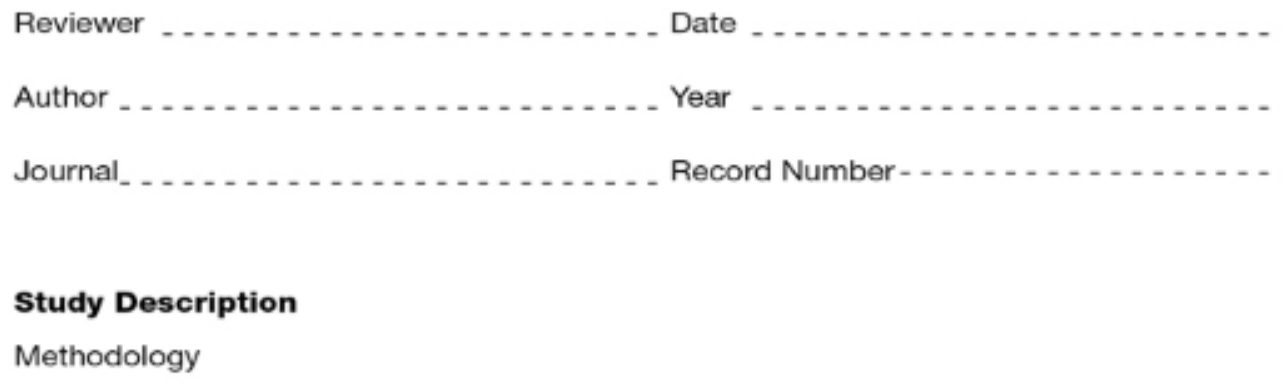




\begin{tabular}{|l|l|l|l|l|}
\hline \multirow{2}{*}{ Findings } & \multirow{2}{*}{$\begin{array}{c}\text { Illustration from } \\
\text { Publication } \\
\text { (page number) }\end{array}$} & Unequivocal & Credible & Unsupported \\
\hline & & & & \\
\hline & & & & \\
\hline & & & & \\
\hline & & & & \\
\hline & & & & \\
\hline & & & & \\
\hline & & & & \\
\hline & & & & \\
\hline & & & & \\
\hline & & & & \\
\hline & & & & \\
\hline & & & & \\
\hline
\end{tabular}

Extraction of findings complete

Yes $\square$

No $\square$ 


\section{Appendix IV: Excluded Studies}

Iedema, R., Allen, S., Sorensen, R., Gallagher, T.H., 2011. What prevents incident disclosure, and what can be done to promote it?

Reason for exclusion: Pilot Final report used to prevent the duplication of results.

Iedema, R., Jorm, C., Wakefield, J., Ryan, C., Dunn, S., 2009. Practicing Open Disclosure: clinical incident communication and systems improvement.

Reason for exclusion: Does not cover phenomena of interest, no disclosing errors to patients.

Iedema, R., Jorm, C.M Wakefield, J., Ryan, C., Sorensen, R. A., 2009. New Structure of Attention? Open Disclosure of Adverse Events to Patients and Their Families.

Reason for exclusion: Final report of Pilot study included to prevent the duplication of results.

Iedema, R., Mallock, N.A., Sorensen, R.J., Manias, E., Tuckett, A.G., Williams, A.F., Perrott, B.E., Brownhill, S.H., Piper, D.A., Hor, S., Hegney, D.G., Scheeres, H.B., Jorm, C.M., 2008. The National Open Disclosure Pilot: evaluation of a policy implementation initiative.

Reason for exclusion: Pilot Final Report included to prevent the duplication of results.

Jeffs, L., Espin, S., Shannon, S. E., Levinson, W., Kohn, M. K., Lingard, L., 2010. A new way of relating: perceptions associated with a team-based error disclosure simulation intervention.

Reason for exclusion: Poor reporting of study findings in this report. 
Jeffs, L., Espin, S., Rorabeck, L., Shannon, S. E., Robins, L., Levinson, W., Gallagher, T.H., Gladkova, O., Lingard, L., 2011. Not Overstepping Professional Boundaries: The Challenging Role of Nurses in Simulated Error Disclosures.

Reason for exclusion: Not clear how the data gave rise to these specific findings.

Kroll, L., Singleton, A., Collier, J., Rees Jones, I., 2008. Learning not to take it seriously: junior doctors' accounts of error.

Reason for exclusion: No data on disclosure of errors to patients.

Luk, L. A., Ng, W. I., Ko, K. K., Ung, V. H., 2008. Nursing management of medication errors

Reason for exclusion: Limited data and analysis reported in this paper.

May, N. and Plews-Ogan, M., 2012. The role of talking (and keeping silent) in physician coping with medical error: A qualitative study.

Reason for exclusion: Methodological structure was not well defined and there was limited data reported.

Schelbred, A. B., and Nord, R., 2007. Nurses' experiences of drug administration errors

Reason for exclusion: Open Disclosure to patients was not the major theme of this study.

Shannon, S.E., Foglia, M.B. , Hardy, M., Gallagher, T.H, 2009. Disclosing errors to patients: Perspectives of registered nurses.

Reason for exclusion: Methodology of study not well reported.

Sorensen, R., Iedema, R., Piper, D., Manias, E., Williams, A., Tuckett A., 2011. Disclosing clinical adverse events to patients: can practice inform policy?

Reason for exclusion: Key elements missing in this report. 
Sorenson, R., Iedema, R., Piper, D., Manias, E., Williams, A., Tuckett, A., 2008.

Health care professionals views of implementing a policy of open disclosure of errors.

Reason for exclusion: Not clear how data gave rise to these specific findings. 